\title{
FINITE TEMPERATURE EXCITATIONS IN A DILUTE BOSE-CONDENSED GAS
}

\author{
by
}

\section{Hua Shi}

A thesis submitted in conformity with the requirements for the Degree of Doctor of Philosophy

Graduate Department of Physics

University of Toronto

(C) Copyright by Hua Shi 1997 
National Library

of Canada

Acquisitions and Bibliographic Services

395 Wellington Street Ottawa ON K1A ON4 Canada
Bibliothèque nationale du Canada

Acquisitions et services bibliographiques

395, rue Wellington

Ottawa ON K1A ON4

Canada
The author has granted a nonexclusive licence allowing the National Library of Canada to reproduce, loan, distribute or sell copies of this thesis in microform, paper or electronic formats.

The author retains ownership of the copyright in this thesis. Neither the thesis nor substantial extracts from it may be printed or otherwise reproduced without the author's permission.
L'auteur a accordé une licence non exclusive permettant à la Bibliothèque nationale du Canada de reproduire, prêter, distribuer ou vendre des copies de cette thèse sous la forme de microfiche/film, de reproduction sur papier ou sur format électronique.

L'auteur conserve la propriété du droit d'auteur qui protège cette thèse. Ni la thèse ni des extraits substantiels de celle-ci ne doivent être imprimés ou autrement reproduits sans son autorisation. 


\title{
FINITE-TEMPERATURE EXCITATIONS IN A DILUTE BOSE-CONDENSED GAS
}

\author{
by Hua Shi
}

Doctor of Philosophy. 199i

Graduate Department of Physics

University of Toronto

\begin{abstract}
In this thesis. we present several approximations applicable to a uniform weaklyinteracting Bose gas at finite temperature. and investigate the excitations and other properties by means of thermal Green's function techniques.

We give a detailed discussion of the first-order Popov approximation. which extends the $T=0$ Bogoliubov approximation by including the Hartree-Fock self-energies of the excited atoms. It leads to a single-particle excitation which is phonon-like in the long wavelength limit. with a velocity which goes as $\sqrt{n_{0}(T)}$. This phonon region collapses as the BEC transition point is approaches. We next discuss the Poporapproximation using a $t$-matrix calculated with self-consistent ladder diagrams. Our analysis shows that this $t$-matrix becomes very temperature dependent as one approaches the transition point, vanishing at $T_{c}$. This is in agreement with the numerical work of Bijlsma and Stoof (1995). who first pointed out that this removes the discontinuous jump in the condensate density at the transition point.

We present a detailed study of the Beliaev-Popov (B-P) approximation for a dilute Bose gas, based on the self-energy diagrams which are second order in the $t$-matrix. We work out the contribution from each individual diagram and give formal expressions for the self-energies and the excitation energy spectrum valid at arbitrary temperatures. We rederive the well-known results of Beliaev at $T=0$. The long wavelength excitations in the Beliaev approximation are phonons with finite lifetimes (damping). We also give the analogous evaluation of the finite temperature B-P selfenergies in the low frequency and long wavelength limit. The excitations are still phonon-like. The corrections near $T_{c}$ to the chemical potential, excitation energy and
\end{abstract}


damping are all found to be proportional to the temperature. As at zero temperature. the finite temperature self-energies contain infrared divergent terms. but these are shown to cancel out in physical quantities in the long wavelength limit. 


\section{ACKNOWLEDGEMENTS}

I thank my supervisor Prof. Allan Griffin for his guidance throughout this work. for his patience with details which often led to better understanding and completely new ideas. for his encouragement during my difficult transition year with my baby girl. for his prompt. careful readings and corrections of all versions of this thesis. and finally for financial support from his research grant.

I have enjoyed discussions with. as well as many courses taught by: various faculty members in this department. I thank in particular Profs. Michael Walker. Rashmi Desai. Bryan Statt. Allan Jacobs. John Sipe. Aephraim Steinberg. and Sajeev John. I also wish to express my deep gratitude towards Dr. H.T.C. Stoof from the Cniversity of L'trecht for his valuable comments and suggestions related to this thesis.

Many other people in this department have helped me directly or indirectly. And many of them have enriched my life as a graduate student by their friendship and inspiring discussions. I thank in particular Wen-Chin Wu. Ya-Wei Hsueh. Wasantha Wijesundera. Jutta Luettmer-Strathman, Daniel Agterberg, Mesfin Woldeyohannes. Puangratana Pairor. Celeste Sagui and Bruce Elrick. I would also like to thank Marianne Khurana and Jennifer Tam for their friendly assistance on many general aspects of my graduate studies. A special thanks goes to the 10 th floor coffee club. which has been an enjoyable place to discuss physics and non-physics matters.

I thank my husband Eric C. Xiao for his everlasting confidence and pride in me as a physicist, for all the happiness he has brought me through the years. Despite of his own challenging job. he has always been very supportive to me. I thank him for taking up a fine father's job with Jasmine when I was occupied. giving me a push when I was down, and cheering with me when I made a little progress. I thank my daughter Jasmine Hongdi and my coming baby for providing a constant source of joy for me. Also, my father and mother have always encouraged and supported me in various ways since my high school years. Their high expectation was part of the power that led to the completion of this work.

My studies have been supported by Sir Run Run Shaw, $\mathrm{U}$ of $\mathrm{T}$ open and departmental fellowships, as well as by research grants from NSERC of Canada. 


\section{Contents}

1 Introduction 1

1.1 History of $\mathrm{BEC}$ studies . . . . . . . . . . . . . . . 2

1.2 Theories of weakly-interacting Bose gases $\ldots \ldots \ldots \ldots$

1.3 Summary of thesis $\ldots \ldots \ldots \ldots \ldots \ldots \ldots$

2 Green's function formalism for a Bose-condensed gas 13

2.1 Imaginary frequency Green s functions . . . . . . . . . . 14

2.2 Bogoliubov prescription for a Bose-condensed system . . . . . . IT

2.3 Dyson-Beliaev equations $\ldots \ldots \ldots \ldots \ldots \ldots$

2.4 Real-time Green s functions $\ldots \ldots \ldots \ldots \ldots \ldots$

2.5 The Bogoliubov approximation $\ldots \ldots \ldots \ldots \ldots$

3 The first-order Popov approximation 31

3.1 The HFB self-energies . . . . . . . . . . . . . . . 32

3.2 The Popor self-energies in terms of the $t$-matrix . . . . . . . 3.5

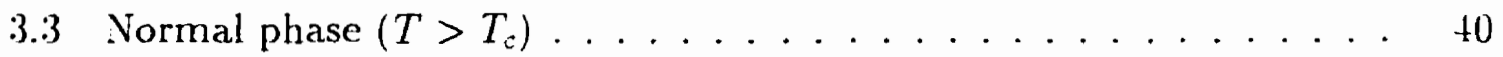

3.4 Bose-condensed phase $\left(T<T_{c}\right) \ldots \ldots \ldots \ldots \ldots \ldots \ldots$

4 The many-body t-matrix approximation $\quad 50$

t.1 The many-body $t$-matrix . . . . . . . . . . . . 51

4.2 Analytical results for the $t$-matrix $\ldots \ldots \ldots \ldots \ldots$

4.3 Numerical results for the $t$-matrix . . . . . . . . . . . . .59

4.4 Assessment of the many-body $t$-matrix approximation . . . . . . 61 
5 The second-order Beliaev-Popov (B-P) approximation 63

5.1 Diagrams and their formal expressions . . . . . . . . . . 6. 6.

5.2 Evaluation of the B-P self-energies ............... 73

5.3 Energy of excitations in the B-P approximation . . . . . . . 79

6 Calculations at $T=0$ using the B-P approximation 83

$6 . \mathrm{T}$ The B-P self-energies at $T=0 \ldots \ldots \ldots$ st

6.2 Quasiparticle spectrum ...................... si

7 Discussion of the $T \neq 0$ B-P approximation $\quad 93$

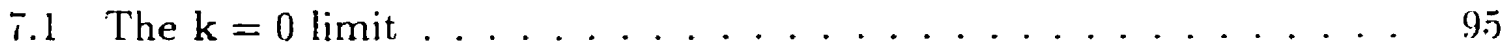

$\tau .2 \quad$ Expansions of $\Sigma^{T}\left(\mathbf{k} . \omega=E_{k}+\delta\right)$ for small $\mathbf{k} \ldots \ldots . . \ldots 8$

¡.3 Damping of phonons at high temperature ........... 104

8 Concluding remarks $\quad 107$

\section{Appendices}

A Scattering theory and the t-matrix for Bose systems $\quad 110$

A.1 Multiple scattering in vacuum ............... 110

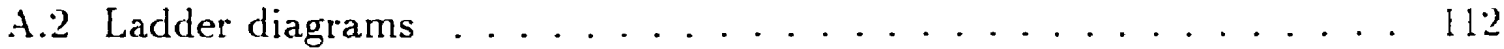

A.3 Connections between $f$ and $[\ldots \ldots \ldots \ldots$

$\begin{array}{lr}\text { References } & 120\end{array}$ 


\section{List of Figures}

2.1 Eight distinct processes involved in the interaction $\hat{V}$. A solid line denotes $\hat{a}$ or $\hat{a}^{\dagger}$, a wiggly line corresponds to a condensate atom or a factor $n_{0}^{1 / 2}$. and a dashed line denotes the interaction $\% \ldots 20$

2.2 Proper self-energies for a Bose-condensed system . . . . . . . . 21

2.3 Green's functions for a Bose-condensed system. . . . . . . . 2.2

2.4 Dyson-Beliaev equations for a Bose-condensed system. . . . . . . . 2:3

2.5 Self-energy diagrams in the Bogoliubov approximation. . . . . . . 28

3.1 Self-energy diagrams in the Popov approximation involving the bare potential v. A solid line with arrow denotes an ideal gas propagator $G^{(0)}$. a wiggly line denotes a condensate atom. and a dash line denotes the interaction $v \ldots \ldots \ldots \ldots \ldots \ldots \ldots \ldots$

3.2 Typical inter-atomic potential $\ldots \ldots \ldots \ldots \ldots \ldots \ldots$

3.3 Diagrammatic definition of the $t$-matrix $\Gamma \ldots \ldots \ldots \ldots$

3.t Self-energy diagrams in the Popov approximation involving $t$-matrix . 38

3.5 Crossover between $n_{0}(T)$ of an interacting gas (solid line) and $n_{0}^{(0)}(T)$ of an ideal Bose gas (dashed line). The changes are exaggerated for clarity. . . . . . . . . . . . . . . . . 46

t.1 Self-energy diagrams in the many-body $t$-matrix approximation. . . 51

4.2 Integral equation for the many-body $t$-matrix $\widetilde{\Gamma}$. (A grey-filled square with four "legs" represents $\tilde{\Gamma}$, a solid line with two arrows represents a "dressed" particle or $G_{11}^{\prime}$, and a dashed line represents the interaction potential.) . . . . . . . . . . . . . . . . 
4.3 Self-consistent $t$-matrix vs. temperature . . . . . . . . . 59

4.4 Condensate density vs. temperature in the many-body $t$-matrix approximation ............................. 60

j.l Diagrams for the diagonal self-energy $\Sigma_{11}$ in the B-P approximation. $\quad 6-1$

$5.2 \quad$ An interaction vertex involving a $t$-matrix . . . . . . . 6. 6

5.3 Diagrams for the off-diagonal self-energy $\Sigma_{12}$ in the B-P approximation 67

5.t Overlap and difference between the many-body $t$-matrix and the B-P

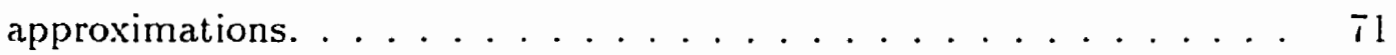

5 Extra diagrams included in the many-body $t$-matrix approximation. . $\quad 7 \cdot 2$

A.I Ladder diagrams that are equally important in the Hartree-Fock ap-

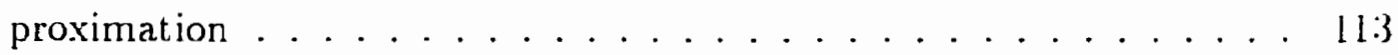

A.2 Diagrammatic definition of the $t$-matrix . . . . . . . . . . $11+$

A.3 Hartree-Fock self-energy diagrams in the t-matrix approximation . . 116 


\section{Chapter 1}

\section{Introduction}

The final achievement of Bose-Einstein condensation (BEC) in a trapped gas of $\mathrm{Rb}$ atoms in 1995 [1] was the result of several decades of research. It also marks the beginning of a whole new area of research into the properties of atomic Bose condensates. This subject is presently undergoing a world-wide explosion of research activity: both experimental and theoretical. As a result, the theory of a weakly-interacting Bose-condensed gas has suddenly become the subject of intense research interest.

The present thesis is motivated by these exciting new developments. even though it deals entirely with uniform weakly-interacting Bose gases while all the recent experimental studies are concerned with Bose gases in an anisotropic parabolic potential well (or trap), i.e. a non-uniform Bose condensate (For reviews, see Refs. [2] and [3]). Our emphasis is on developing an understanding of the elementary excitations of a uniform Bose-condensed gas at finite temperatures, where a substantial fraction of the atoms are no longer in the lowest energy single-particle state (the Bose condensate). In contrast to the well-understood situation at $T=0$. where all but a few percent of the atoms are in the condensate, very few theoretical studies have been concerned with weakly-interacting gas with a strongly-depleted condensate. We believe our results in this thesis are the first step to understanding the analogous questions in the trapped atomic gases of current interest. 


\subsection{History of BEC studies}

It is useful to first give a brief history of BEC studies. starting with the initial prediction of Einstein in 1925 [4] for the case of a non-interacting gas of Bose atoms ( $A$ Bose atom has a net integer spin. All atoms with an even number of neutrons satisfy Bose statistics. which accounts for about $7.5 \%$ of the atoms in the periodic table). Bose-Einstein condensation in an ideal gas is a standard topic in all textbooks on statistical mechanics [5].

The momentum distribution of an ideal uniform Bose gas is given by

$$
f_{B}\left(\epsilon_{\mathbf{k}}\right)=\frac{1}{e^{3\left(\epsilon^{-} \mathbf{k}^{-\mu)}\right.}-1}
$$

where $\epsilon_{\mathbf{k}}=\hbar^{2} k^{2} / 2 m .3=1 / k_{B} T$. and $\mu$ is the chemical potential determined by the condition that the sum over all possible states equals the total number of particles $v$. In a system of macroscopic size. the sum can be converted into an integral

$$
N=\sum_{\mathbf{k}} f\left(\epsilon_{\mathbf{k}}\right)=\int D(\epsilon) f(\epsilon) d \epsilon .
$$

where the density of states $D(\epsilon)$ goes as $\sim \sqrt{\epsilon}$. If the volume $V$ and the particle number. are fixed. the (negative) chemical potential $\mu$ increases as the temperature $T$ decreases. At a certain temperature $T_{0} \cdot \mu$ reaches zero, the maximum value it can reach in an ideal Bose gas, and thus the integral in (1.2) gives

$$
V=2.612 \frac{V}{\lambda^{3}\left(T_{c}\right)},
$$

where the thermal de Broglie wavelength $\lambda(T)$ is

$$
\lambda(T) \equiv\left(\frac{2 \pi \hbar^{2}}{m k_{B} T}\right)^{1 / 2} .
$$

One may view $T_{c}$ defined by (1.3) as equivalent to the condition that the thermal de Broglie wavelength $\lambda(T)$ becomes comparable to the average separation $d$ between 
particles $\left(d \sim n^{-1 / 3}\right)$. In this case. the quantum mechanical wave nature of the particles leads to all atoms becoming increasingly correlated. This is the essential physics behind $\mathrm{BEC}$ in an ideal Bose gas.

As $T$ goes lower than $T_{c}, \mu$ is "pinned" at zero. and the right hand side of (1.3) becomes smaller than $N$. As Einstein [4] first pointed out, the additional particles enter the ground state to form what is now called the Bose-Einstein condensate. The number of particles in this lowest state is given by

$$
V_{0}=N-2.612 \frac{V}{\lambda^{3}(T)}>0 . \quad \text { if } T<T_{i}
$$

From (1.3) and (1.5). one obtains the temperature-dependent condensate fraction

$$
\frac{\vee_{0}(T)}{. V}=1-\left(\frac{T}{T_{c}}\right)^{3 / 2} \text {. }
$$

It is immediately seen that $V_{0}=. V$ at $T=0$. that is. all the atoms are in the lowest single-particle state $(k=0)$.

To summarize. Bose-Einstein statistics described by (1.1) predicts that at a finite temperature given by (1.3). the lowest state becomes macroscopically occupied and a transition occurs to a new phase of matter characterized by the condensate fraction. It is the only phase transition in condensed matter physics that can occur in the absence of interactions. being entirely due to quantum mechanical effects related to Bose statistics.

At the time of Einstein's prediction. the nature of second-order phase transitions was not yet understood, and Uhlenbeck criticized Einstein's analysis in his thesis in 192 $\tau$. It was only in 1937 that it was generally agreed that Uhlenbeck's objections to a phase transition in an ideal Bose gas were incorrect. Around the same time. superfluidity had been just discovered in liquid ${ }^{4}$ He below $2.17 \mathrm{~K}$. Fritz London [6] immediately made the bold conjecture that this superfluidity was related to the form of Bose-Einstein condensation, suitably generalized to a liquid. The basis of London's argument was that ${ }^{4} \mathrm{He}$ was a Bose atom $(S=0)$ and moreover if liquid 
${ }^{4} \mathrm{He}$ was treated as an ideal gas. with the density $2.20 \times 10^{22}$ atoms $/ \mathrm{cm}^{3}$. its BEC transition temperature given by $(1.3)$ would be $3.15 \mathrm{~K}$. This is remarkably close to the observed superfluid transition temperature. Essentially London and Tisza argued that the superfluid characteristics were related to the motion of the Bose condensate. moving as a whole. The development and confirmation of these ideas about superfluid ${ }^{4}$ He took many years. Indeed. it has been controversial since the very successful Landau theory [7] of the low temperature properties of superfluid the (based on a phenomenological model of a weakly-interacting gas of phonon-roton excitations) made no explicit reference to any role of the Bose condensate.

Our current understanding of superfluid ${ }^{4} \mathrm{He}$ as a Bose-condensed liquid has been extensively reviewed in a monograph by Griffin [8]. to which we refer the reader. Some key theoretical papers (all at $T=0$ ) are:

(a) The work of Bogoliubov [9]. who showed the connection between a Bose condensate and a phonon-like dispersion relation for long wavelength elementary excitations. This paper was restricted to a weakly interacting Bose gas but later it was realized that it already captured many general features of Bose-condensed systems.

(b) The work of Penrose and Onsager [10]. who showed how to formulate in a precise way the concept of Bose-condensation in any interacting Bose system (gas or liquid). even when the condensate fraction was small (strongly depleted).

(c) The general field-theoretical formulation of Beliaev [11] of how to work out the properties of a Bose-condensed system in the presence of a Bose condensate. Beliaev's work also first emphasized the key role of the phase of the Bose order parameter and its relation to the superfluid velocity.

These papers laid the foundation for intensive theoretical work on the general theory of interacting Bose-condensed system in the period 19.58-196.5. The motivation was always to understand the excitations and other properties of superfluid ${ }^{4} \mathrm{He}[8]$. but the hypothetical case of a dilute weakly-interacting Bose gas was often considered as an 
illustrative example of the very complex calculations. Since that period, the Green's function treatment of a weakly-interacting Bose-condensed gas at $T=0$ has become one of the standard topics in textbooks on many-body theory [12.13. 14]. However. to a large extent. this topic has only been of research interest to a small number of many-body theorists. One of the reasons is that, apart from some general relations. it is very difficult to carry out quantitative calculations for superfluid ${ }^{4} \mathrm{He}$. In a sense. the characteristic features of the Bose condensate and Bose broken symmetry were hard to extract from the complicated dynamics which arise in any liquid.

In view of the difficulties in dealing with a strongly interacting. dense system like superfluid 'He. it is not surprising that since 1960's. there has been an increasing experimental effort to achieve BEC in a dilute Bose gas. At the low temperature needed for BEC. most systems will form condensed phases (liquid or solid) due to the attractive inter-atomic interactions. Thus one requires conditions that allow Bose condensation to occur rapidly, relative to the longer time scales needed for the competing phase changes.

One of the most extensively studied candidates for BEC has been spin-aligned hydrogen (For an authoritative review. see Greytak in [3]). By magnetically aligning the spins of the hydrogen atoms, one obtains a system that remains a gas at even $T=0$. Moreover. its density can be varied over several orders of magnitude. ranging from a weakly to strongly interacting Bose gas, and with the possibility of $\mathrm{BEC}$. So far (199i), experiments have not produced a Bose condensate in this system. due to the increasing tendency towards recombination to molecular hydrogen $\mathrm{H}_{2}$ (due to three-body collisions involving spin flips) as the density needed for BEC is approached. However. in the last decade or so, the study of spin-polarized hydrogen stimulated much theoretical and experimental interest in achieving $\mathrm{BEC}$ in an atomic gas.

Another system as a candidate for BEC has been excitons in optically pumped semiconductors (For a review, see Wolfe et al in [3]). Excitons (a bound state of an electron and a hole) move like a weakly-interacting gas in the crystal. Because of the small exciton mass (on the order of the mass of an electron), the BEC transition temperature of exciton systems can be as high as $\sim 100 \mathrm{~K}$ if the density of the exciton 
gas is $10^{19} \mathrm{~cm}^{-3}$ (easily achievable with laser pulses). Excitons have a finite lifetime due to recombination. but in high purity $\mathrm{C}^{\prime} \mathrm{u}_{2} \mathrm{O}$ crystals. these lifetimes can be as long as $10^{-3} \mathrm{~s}$. which are enormous compared to other relaxation times $\left(\sim 10^{-8} \mathrm{~s}\right)$ involved. While this finite exciton lifetime is a complication. the resulting decay luminescence turns out to be a very direct measure of the kinetic energy of the decaying exciton and hence can be used to detect the presence of an exciton Bose condensate (excitons with zero kinetic energy). The theory of this decay spectrum has been worked out by Shi. Verechaka and Griffin [1.5]. In 1993. Lin and Wolfe [16] announced the first convincing evidence of BEC transition of paraexcitons $(S=0)$ in a stressed high quality $\mathrm{Cu}_{2} \mathrm{O}$ crystal. However, due to the current poor knowledge of excitonexciton interactions. and the complications due to various recombination processes and exciton-phonon interactions, the theoretical analysis of the decay luminescence data is quite complicated and much work remains to be done [15. 1i]. In passing. we note that the work of Shi et al [1.5] shows that the decay luminescence is a direct probe of the single-particle Green's function of a Bose gas. Very few experimental techniques do this.

The search for $\mathrm{BEC}^{\prime}$ in a dilute gas of alkali atoms has also been actively pursued for about a decade [2]. In these experiments. alkali atoms are first cooled and trapped by laser beams. then loaded into a magnetic trap. where a selection of hyperfine spin state is made so that all the atoms in the trap have the same spin. The gas is then further cooled by an evaporation technique. involving selective of pulses which flip the spins of atoms with relatively high kinetic energy, which are then expelled from the trap. Due to a large scattering cross section. the remaining atoms quickly rethermalize at a lower temperature. populating the lower levels of the parabolic well. Therefore, one obtains a lower temperature and higher density (i.e. at the center of the trap) at the same time. The first successful observation of BEC was made with ${ }^{8 \pi} \mathrm{Rb}$ atoms [1] (which have 87 nucleons and 37 electrons, and thus obey Bose statistics) at temperatures of the order of $10^{-7} \mathbf{K}$. Shortly after, BEC condensates were also produced using ${ }^{23} \mathrm{Na}$ atoms [18] with much larger numbers of atoms $\left(\sim 10^{6}\right)$ and hence a higher transition temperature $\left(\sim 10^{-6} \mathrm{~K}\right)$. This field of research is expanding 
very rapidly at the present time.

\subsection{Theories of weakly-interacting Bose gases}

With this brief history in mind. one can understand the special excitement among theorists when BEC was finally achieved in a gas of atoms. Suddenly all the theoretical calculations on dilute Bose gases over the last 40 years had a direct significance. rather than as tentative steps to understanding liquid ${ }^{4} \mathrm{He}$. Moreover. the experiments involve lowering the temperature of the trapped atomic gases through the BEC transition to well below $T_{c}$. As a result, these new experimental studies have also emphasized the need to understand the finite temperature properties of Bosecondensed systems. With few exceptions, most of the available theoretical literature on Bose gases is restricted to $T=0$.

As we have noted earlier, the theory of excitations at $T=0$ is simple because one can assume that all atoms are in the condensate and hence the single-particle excitations only involve atoms going in and out of this condensate. This problem was first solved by Bogoliubov [9] for a uniform gas (It is straightforward to extcnd this theory to a trapped Bose gas [19]. The latter is usually referred to as the timedependent Gross-Pitaevskii theory [20. 21]). In his classic paper. Bogoliubor [9] presented a theory of a dilute weakly interacting Bose gas at temperatures far below the transition temperature.

A major result of Bogoliubov's calculation was that only a small fraction of the atoms were removed from the condensate at $T=0$ due to interactions. Specifically: the number of particle in the condensate, $N_{0}$, is given by

$$
N_{0}=N\left[1-\frac{8}{3}\left(\frac{n a^{3}}{\pi}\right)^{1 / 2}\right] \text {, }
$$

where $n=N / V$ is the density and $a$ is the s-wave scattering length. For a dilute Bose gas, we have $a \ll n^{-1 / 3}$ or $n a^{3} \ll 1$. In spite of this small interaction-induced "depletion", the Bogoliubov model shows the interactions in the presence of a conden- 
sate lead to an acoustic (or phonon) excitation spectrum at long wavelengths. Wore precisely. Bogoliubov found that for wavevector $k \ll m c / \hbar$. the excitation energy is given by

$$
E_{k} \simeq \hbar c k \text {. }
$$

with the phonon velocity

$$
c=\left(\frac{4 \pi \hbar^{2} a}{m} \frac{n}{m}\right)^{1 / 2} .
$$

Obviously, this phonon spectrum collapses if $a=0$. All of the low-temperature thermodynamics of a Bose-condensed system follow from this phonon dispersion relation. Gavoret and Nozières [2-2] were the first to show at $T=0$ that to all orders in perturbation theory. a Bose-condensed system always exhibited such a phonon spectrum with a speed given by usual thermodynamic derivative.

Bogoliubov's calculation also introduced the seminal idea of treating the atoms in the condensate classically. He noted that for atoms in the $k=0$ state. the Bose commutation relation gave

$$
\hat{a}_{0} \hat{a}_{0}^{\dagger}-\hat{a}_{0}^{\dagger} \hat{a}_{0}=1
$$

However, since the expectation values of the two terms on the left hand side are each of the order of $V_{0}$. which is a very large number. Thus to an extremely good approximation, the operators of the $\mathbf{k}=0$ state commute with each other (as well as with all other $\mathbf{k} \neq 0$ operators). Therefore they can be treated as c-numbers. with $\hat{a}_{0}=\hat{a}_{0}^{\dagger}=\sqrt{\hat{V}_{0}}$. In this Bogoliubov prescription, the condensate acts like a classical particle reservoir, which non-condensate atoms can enter and leave via scattering [11]. Thus the number of atoms is no longer a constant of motion. As an immediate consequence, one needs to include anomalous propagators (Green's functions) representing two particles going into or out of the condensate. This approach forms the basis of the systematic application of quantum field theory to an interacting system of bosons due to Beliaev [11] in 1958, which was developed further by Hugenholtz and Pines [23], and by Gavoret and Nozières [22]. This led to a generalized Green's function formalism which built in the crucial role of the Bose condensate, and allowed one to 
determine the general characteristics of the system, such as the excitation spectrum. the momentum distribution of atoms, etc. After these pioneer work. this field of study. has been extensively developed and extended [\$].

The first finite temperature calculations for an interacting Bose gas were made by Lee. Huang and Yang [24], by means of a pseudopotential method for a gas of hard spheres. For the low energy scattering. the details of the potential are not important and can be shown to be described by s-wave. The potential can be treated as an effective zero-range interaction

$$
v\left(\mathbf{x}-\mathbf{x}^{\prime}\right)=\frac{4 \pi \hbar^{2} a}{m} \delta\left(\mathbf{x}-\mathbf{x}^{\prime}\right)
$$

In a hard-sphere gas. the scattering length $a$ equals to the diameter of the atoms. Lee. Huang and Yang carried out calculations near $T_{c}$ to first order in the interaction assuming

$$
a \ll \lambda(T), \quad a \ll n^{-1 / 3} .
$$

where $\lambda(T)$ is the thermal de Broglie wavelength given by (1.t). For a review of the thermodynamic properties of a Bose gas near $T_{c}$ to first order in $a$. see Ref.[25].

Following the work at $T=0$. many people also developed the analogous fieldtheoretic approach applicable at finite temperatures. We mention here specifically the papers of Hohenberg and Martin [26], Popov and Faddeev [27]. Singh [28]. and Cheung and Griffin [29]. In principle, these allow one to calculate the properties of an interacting Bose system. such as the thermodynamic potential. specific heat. condensate density. etc. As at $T=0$, such finite $T$ calculations are complicated by the subtle role of correlations induced by the Bose-broken symmetry. Moreover. even in a dilute gas. the finite $T$ case is difficult because the thermally-induced depletion fraction is now large. One has to be careful in treating the condensate and excited atoms in a consistent fashion.

Another well-known difficulty is the fact that even for regular repulsive interactions, perturbation theory for Bose-condensed systems diverges at small momenta $[22,30]$. That is to say, certain terms in the perturbation series are singular for 
k. $u \rightarrow 0$. a result which can be traced back to the fact that the single-particle excitations are phonon-like. These singularities have to be handled with care in order to obtain correct final results. Fortunately. the infrared divergences appear to cancel out in all physical quantities [22, 30].

One major omission of the present thesis is any discussion of the relation between the single-particle excitations (which we calculate) and the density fluctuation spectrum. The Bose broken symmetry is known (see Chapter 5 of Ref. [8]) to lead to the equivalence of these two kinds of excitations and this imposes a very strong constraint on approximations [26].

\subsection{Summary of thesis}

The present thesis is devoted to a systematic study of the finite temperature excitations. using the Beliaer Green's function formalism. Our emphasis is on how to include the effects of the non-condensate atoms and is based on the second-order Beliaev-Popov self-energy diagrams. For clarity. we should note that while our calculations are based on second-order Dyson-Beliaev self-energies. we use renormalized propagators, and thus these self-energies really involve terms to all orders in the interaction. As with any many body calculation based on a selection of self-energy diagrams which involve self-consistent propagators. it is sometimes difficult to give convincing arguments about their relative importance. We have tried to do this as best as we are able to at the present time. following mainly the arguments given by Popov [31].

The thermal Green's function method is briefly reviewed in Chapter 2. where we give the Dyson-Beliaev equations expressing the $2 \times 2$ matrix Green's function in terms of the two kinds of self-energies. For illustration, we use this formalism to discuss the simple $T=0$ Bogoliubov model (this was first done by Beliaev [11] and is discussed in most many-body textbooks [12, 13]).

These techniques are then applied to a dilute Bose gas at finite temperature in Chapter 3, using the first-order Hartree-Fock approximation to deal with the inter- 
actions between the excited atoms plus the Bogoliubov approximation to account for interactions with the condensate, as first suggested by Popor [32]. Calculating the poles of the single-particle diagonal and off-diagonal Green's functions, we find the quasiparticle energy spectrum has a structure similar to that of the $T=0$ Bogoliubov model. but now with a temperature-dependent condensate. This leads to the collapse of the low $k$ phonon-spectrum to free particles as $T \rightarrow T_{c}$. Like many other non-self-consistent first-order calculations. the Popov approximation leads to a jump in the condensate density at the transition point. and thus predicts a first-order phase transition. Following recent work of Bijlsma and Stoof [3:3]. in Chapter 4 we modify the Popov approximation by using a self-consistent $t$-matrix. rather than the ordinary one using unperturbed propagators. This self-consistent $t$-matrix is found to be very temperature-dependent. and vanishes at $T_{c}$. It leads to a smooth change of the condensate density at the $\mathrm{BEC}$ transition point.

In Chapter 5 . we discuss the more complicated second-order Beliaer-Popor approximation. and work out the formal expressions of the self-energies and their relations to the single-particle excitation energy. ('sing these results. Bcliaer's sccondorder calculations at $T=0$ are re-derived in Chapter 6 . We evaluate the self-cnergies near the poles $\lrcorner \sim E_{k}$ (where $E_{k}$ is the Bogoliubov excitation energy of Chapter 2 ) and expand our results in powers of $h$ in the long wavelength limit. Our explicit expressions of the self-energies involve infrared divergent terms. We show that these divergent terms cancel out exactly (as expected), leading to well-defined physical quantities. such as the quasiparticle energy and the single-particle spectral density: As expected. the energy spectrum of excitations in the Beliaev approximation is phonon-like in the long wavelength limit. It also contains an imaginary part (damping) which goes as $\sim k^{5}$. These second-order Beliaev self-energies are the lowest approximation which involves damping of excitations. While all the final results of Chapter 6 are known, we hope our very detailed analysis will make Beliaev's work [34] more accessible.

The analogous evaluation of our expressions for the finite-temperature BeliaevPopov self-energies at small $\mathrm{k}$ and $\omega$ is much more difficult than in the $T=0$ case. 
By expanding the self-energies in powers of $k$ at $\omega \sim E_{k}$ (where $E_{k}$ is now the Popov excitation energy of Chapter 3) as in the zero-temperature case. we are taking the limit $\omega \rightarrow 0$ and $k \rightarrow 0$ at the same time. This is not appropriate at finite temperature because the additional thermal scattering terms involved in the self-energies require that one take the limit $\mathbf{k} \rightarrow 0$ first. To overcome this difficulty: in Chapter $i$ we introduce a small gap between the frequency and the Popov quasiparticle energy and expand the self-energy at $\omega=E_{k}+\delta$, rather than at $\omega=E_{k}$. This gap $\delta$ is kept finite until the expansion in powers of $k$ is done. We thus obtain the expansion for the self-energies in powers of $k$ at finite $T$. These results allow us to calculate the single-particle excitation energy and damping, as well as the chemical potential by means of the Hugenholtz-Pines theorem [2:3].

We believe the results will be of considerable interest to the growing $\mathrm{BEC}$ community. who are interested in the analogous problems for trapped Bose gases. We have made an attempt, in an admittedly highly mathematical development. to discuss the physics involved in the diagrammatic analysis as well as to describe the intermediate steps in some detail. Without denying the brilliance of the papers by Beliaev at $T=0$ [34] and Popov at finite temperatures close to the transition [31. 32]. their accounts are written in a very terse style with many important features left tor the reader to figure out. The present thesis is also a status report of our current understanding of excitations in uniform Bose gases at finite temperature. We hope that it will provide the basis for future theoretical studies which will clarify several questions raised in the thesis, as well as in generalizations to deal with excitations in trapped Bose gases. 


\section{Chapter 2}

\section{Green's function formalism for a}

\section{Bose-condensed gas}

In this chapter. we briefly review the finite-temperature (or thermal) Green's function formalism. This technique is the most effective way of calculating the equilibrium thermodynamic properties. as well as single-particle excitations of the system. Although this chapter does not contain anything new, it is included for the convenience of the reader and to introduce notation. In the Bose-condensed phase characterized by a macroscopic number of atoms in the zero-momentum single-particle state. it is convenient to regard the operators $\hat{a}_{0}$ and $\hat{a}_{0}^{\dagger}$ for this state as c-numbers. This procedure. called Bogoliubov prescription, is discussed in Section 2.2. Within this broken symmetry phase. one deals with anomalous averages and it is also necessary to introduce (Section 2.3) a $2 \times 2$ matrix Green's function or propagator. Its diagonal elements are the normal Green's functions, and off-diagonal elements are called the anomalous Green's functions. We write down the Dyson-Beliaev equations for a Bose-condensed system $[11,28]$, which give different kinds of Green's function in terms of the $2 \times 2$ matrix self-energy. In Section 2.t, we review how the thermal Green's function at imaginary frequencies are related to the real time Green's function by a simple analytic continuation, in which the single-particle spectral density function plays a central role. Finally, in Section 2.5, we illustrate this formalism by considering the Bogoliubov model approximation at $T=0$. 


\subsection{Imaginary frequency Green's functions}

The system we are concerned with consists of.$V$ atoms obeying Bose statistics. enclosed in a box of volume $V$ and interacting through the two-body potential $\iota \cdot\left(\mathbf{x}-\mathbf{x}^{\prime}\right)$. For simplicity, the atoms are assumed to have zero net spin. For a Bose-condensed system. it is particularly convenient to use the grand canonical ensemble. which allows for the possibility of a variable number of particles. In the second quantized formalism. the grand canonical "Hamiltonian" of the system is given by

$$
\begin{aligned}
\hat{K} \equiv & \hat{H}-\mu \hat{v} \\
= & \int d \mathbf{x} \hat{\psi}^{\dagger}(\mathbf{x})\left[-\frac{\hbar^{2}}{2 m} \nabla^{2}-\mu\right] \hat{\dot{\psi}}(\mathbf{x}) \\
& +\frac{1}{2} \int d \mathbf{x} \int d \mathbf{x}^{\prime} \hat{\psi}^{\dagger}(\mathbf{x}) \hat{\psi}^{\dagger}\left(\mathbf{x}^{\prime}\right) v\left(\mathbf{x}-\mathbf{x}^{\prime}\right) \hat{\psi} \cdot\left(\mathbf{x}^{\prime}\right) \hat{\psi}(\mathbf{x}) .
\end{aligned}
$$

Here $\dot{\psi}(\mathbf{x}) \cdot \dot{\psi}^{\dagger}(\mathbf{x})$ are the boson field operators. satisfying the usual Bose commutation relations

$$
\left[\hat{i} \cdot(\mathbf{x}) \cdot \hat{\iota}\left(\mathbf{x}^{\prime}\right)\right]=0 . \quad\left[\hat{\varphi}(\mathbf{x}) \cdot \hat{u^{\prime}}\left(\mathbf{x}^{\prime}\right)\right]=\delta\left(\mathbf{x}-\mathbf{x}^{\prime}\right)
$$

The chemical potential $\mu$ is chosen so that $\langle\hat{V}\rangle=. V$. With the grand canonical Hamiltonian $\hat{K}$. we introduce the modified $\tau$-dependent Heisenberg picture for any Schrödinger operator $\hat{O}(\mathbf{x})$ (see standard texts on many-body sustems such as Refs.[12, 13. 3.5]):

$$
\hat{O}(\mathbf{x} \tau) \equiv \epsilon^{\dot{K} \tau} \hat{O}(\mathbf{x}) \epsilon^{-\dot{K} \tau}
$$

In particular. the field operators have a $\tau$-dependence given by

$$
\begin{aligned}
\hat{\psi}(\mathbf{x} \tau) & =e^{\hat{K} \tau} \hat{\psi}(\mathbf{x}) e^{-\dot{K}^{\dagger} \tau}, \\
\hat{\psi}^{\dagger}(\mathbf{x} \tau) & =e^{\dot{K} \tau} \hat{\dot{\psi}}^{\dagger}(\mathbf{x}) e^{-\hat{K}^{\dagger} \tau} .
\end{aligned}
$$

Comparing this representation with the standard real-time representation. the variable $\tau$ can be viewed as an "imaginary" time $\tau=i t / \hbar$. 
The single-particle thermal (or imaginary time) Green's function is defined as

$$
\begin{aligned}
G\left(\mathbf{x} \tau, \mathbf{x}^{\prime} \tau^{\prime}\right) & \equiv-\operatorname{Tr}\left[\epsilon^{3(\Omega-\hat{K})} T_{\tau} \hat{\psi}(\mathbf{x} \tau) \hat{\psi}^{\dagger}\left(\mathbf{x}^{\prime} \tau^{\prime}\right)\right] \\
& =-\left\langle T_{\tau} \dot{\psi}(\mathbf{x} \tau) \dot{\psi^{\dagger}}\left(\mathbf{x}^{\prime} \tau^{\prime}\right)\right\rangle
\end{aligned}
$$

where $3=1 / k_{B} T$ and $T_{+}$is a $\tau$ ordering operator. which arranges the smallest $\tau$ to the right. The grand canonical ensemble average is denoted by

$$
\langle\cdots\rangle \equiv \operatorname{Tr}\left[e^{3(\Omega-\dot{K})} \cdots\right]
$$

where $\Omega$ is the thermodynamic potential. defined as

$$
\epsilon^{-3 \Omega} \equiv \operatorname{Tr}\left(\epsilon^{-i \beta \hat{K}}\right)
$$

For most purposes. it is more convenient to work with the single-particle momentum representation. Assuming periodic boundary conditions, the operators $\dot{i}(\mathbf{x})$. $\hat{L}^{\dagger}(\mathbf{x})$ can be written in the single-particle momentum representation as

$$
\dot{u}(\mathbf{x})=\frac{1}{\sqrt{l}} \sum_{\mathbf{k}} \hat{a}_{\mathbf{k}} \epsilon^{i \mathbf{k} \cdot \mathbf{x}} . \quad \dot{\varphi}^{\dagger}(\mathbf{x})=\frac{1}{\sqrt{l}} \sum_{\mathbf{k}} \hat{a}_{\mathbf{k}}^{\dagger} \epsilon^{-i \mathbf{k} \cdot \mathbf{x}}
$$

where $\hat{a}_{\mathbf{k}}$, $\hat{a}_{\mathbf{k}}^{\dagger}$ are the Bose annihilation and creation operators. respectively: for the single-particle state of momentum $\mathbf{k}$. From (2.2) follows the Bose commutation rules for $\hat{a}_{\mathbf{k}}$ and $\hat{a}_{\mathbf{k}}^{\dagger}$ :

$$
\left[\hat{a}_{\mathbf{k}}, \hat{a}_{\mathbf{k}^{\prime}}\right]=0, \quad\left[\hat{a}_{\mathbf{k}:} \hat{a}_{\mathbf{k}^{\prime}}^{\dagger}\right]=\delta_{\mathbf{k} \cdot \mathbf{k}^{\prime}}
$$

In the single-particle momentum representation, we may write $C_{f}\left(\mathbf{x} \tau \cdot \mathbf{x}^{\prime} \tau^{\prime}\right)$ in (2.6) as:

$$
G\left(\mathbf{x} \tau, \mathbf{x}^{\prime} \tau^{\prime}\right) \equiv-\frac{1}{V^{\prime}} \sum_{\mathbf{k}, \mathbf{k}^{\prime}} \epsilon^{i\left(\mathbf{k} \cdot \mathbf{x}-\mathbf{k}^{\prime} \cdot \mathbf{x}^{\prime}\right\rangle}\left\langle T_{\tau} \hat{a}_{\mathbf{k}}(\tau) \hat{a}_{\mathbf{k}^{\prime}}^{\dagger}\left(\tau^{\prime}\right)\right\rangle
$$

In the case of a uniform system governed by a time-independent Hamiltonian. $C_{i}$ depends only on the differences $\mathbf{x}-\mathbf{x}^{\prime}$ and $\tau-\tau^{\prime}$ (see standard many-body texts such 
as $\operatorname{Ref} .[13])$, with

$$
G(\mathbf{x}, \tau)=\frac{1}{V} \sum_{\mathbf{k}} e^{\mathbf{i} \cdot \mathbf{x}} G_{r}(\mathbf{k} . \tau)
$$

where

$$
G(\mathbf{k} . \tau) \equiv-\left\langle T_{\tau} \hat{a}_{\mathbf{k}}(\tau) \hat{a}_{\mathbf{k}}^{\dagger}(0)\right\rangle
$$

It is easy to verify that for bosons, $G($ k. $\tau$ ) satisfies the famous periodic condition

$$
G(\mathbf{k} \cdot \tau)=C(\mathbf{k} \cdot \tau+3)
$$

for $-3<\tau<0$. where the right-hand side of the equation gives the Cireen $\mathrm{s}$ function for $0<\tau+3<3$. This periodicity of $G(\mathbf{k}, \tau)$ in the variable $\tau$ with period 3 leads immediately to the following Fourier expansion:

$$
\begin{aligned}
G(\mathbf{k} . \tau) & =\frac{1}{3} \sum_{i=\omega_{n}} e^{-i \omega_{n} \tau} C_{i}\left(\mathrm{k} . i \omega_{n}\right) . \\
G\left(\mathbf{k}, i \omega_{n}\right) & =\int_{0}^{3} d \tau \epsilon^{i \omega_{n} \tau} C_{i}(\mathrm{k} . \tau) . \\
\omega_{n} & =\frac{\pi n}{3}, \quad n=0 . \pm 2 . \pm 4 . \cdots
\end{aligned}
$$

Here $\omega_{n}$ is a Bose Matsubara frequency (which has the dimension of energy in our notation). and because of Bose statistics. the integer $n$ is restricted to even values. The Fourier component $G_{r}\left(\mathbf{k} \cdot i \omega_{n}\right)$ is a function of the discrete Matsubara frequencies that are evenly distributed on the imaginary frequency axis. At $T \rightarrow 0$. we note that the spacing between the discrete Matsubara frequencies goes to zero.

The thermal Green's function defined in (2.6) can be used to evaluate the equilibrium thermodynamic properties of the system, such as the number of particles with a certain momentum. By definition (see. for example, p.229 of [1:3]),

$$
\begin{aligned}
\left\langle\hat{n}_{\mathbf{k}}\right\rangle \equiv\left\langle\hat{a}_{\mathbf{k}}^{\dagger} \hat{a}_{\mathbf{k}}\right\rangle & =\left\langle T_{\tau} \hat{a}_{\mathbf{k}}(\tau) \hat{a}_{\mathbf{k}}^{\dagger}\left(\tau^{+}\right)\right\rangle \\
& =-\frac{1}{3} \sum_{i \omega_{n}} e^{i \omega_{n} \eta} G\left(\mathbf{k} \cdot i \omega_{n}\right),
\end{aligned}
$$

where $\tau^{+}$denotes the limiting value $\tau+\eta$ as $\eta$ approaches zero from positive values. 
The mean number of particles in the system is then given by

$$
V(T, V, \mu)=V_{0}-\frac{V}{3} \int \frac{d \mathbf{k}}{(2 \pi)^{3}} \sum_{i \omega_{n}} \epsilon^{i \omega_{n} \eta} C\left(\mathbf{k} \cdot i \omega_{n}\right)
$$

The ensemble average of any single-particle operator. as well as the mean potential energy is expressible in terms of $G$. Moreover. one can also relate the thermodynamic potential $\Omega(T . V \mu)$ to the single-particle thermal Green's function $C_{r}$ [1:3. 28]. which enables us to calculate the general equilibrium thermodynamic properties of the system.

The excited states of the system containing $. \dot{V} \pm 1$ particles are related to the poles of the real-time Green's function [13. 36]. At finite temperature. however. the real-time Green's function involves a very complicated perturbation expansion. In contrast. the thermal Green's function as defined here has a simpler perturbation expansion. similar to that for the $T=0$ Green's function. Fortunately, as we shall discuss in Section 2.t. the Fourier transforms of the thermal Green's function and the real-time Green's function can be uniquely related to each other through a simple analytic continuation. Thus the imaginary frequency (or thermal) Cireen s function can be used to calculate the single-particle excitations of the system.

\subsection{Bogoliubov prescription for a Bose-condensed system}

In the preceding section. we have defined the single-particle temperature Green's function. The remaining task is to calculate the Green's function $G$. For a Bose system. this task is complicated by the possible phase transition to a Bose-condensed state. in other words, by the spontaneous symmetry-breaking below a certain temperature $T_{c}$. A more rigorous way of dealing with the new phase is to explicitly include a symmetry-breaking term in the Hamiltonian (2.I),

$$
-\frac{1}{2} \int d \mathbf{x}\left[\nu^{*} \hat{\psi}(\mathbf{x})+\nu \hat{\psi}^{\dagger}(\mathbf{x})\right]
$$


where $\nu$ is the Bose symmetry breaking field. The spontaneous symmetry breaking and a new condensed phase is signaled by the non-vanishing of the average [11. 26]

$$
\langle\dot{u}(\mathbf{x})\rangle=\Phi_{0}(\mathbf{x}) \neq 0 .
$$

even in the limit of a zero off-diagonal field $(\nu \rightarrow 0)$. We refer to the thesis of Talbot [37] for a detailed discussion of Green's function in a Bose system where the symmetry. breaking field is kept throughout. In this thesis. however, we will assume the limit of $\nu \rightarrow 0$ and leave this term implicit.

$\Phi_{0}(\mathbf{x})$ in $(2.21)$ is often referred to as the macroscopic wavefunction of the condensate and in general has an amplitude and a phase. In a uniform system and in the absence of any supercurrent, we can take $\Phi_{0}(\mathbf{x})$ to be real and independent of position. In this case. $\Phi_{0}$ equals to square root of the condensate density $n_{0}$. We are then led to separate the boson field operator into two parts:

$$
\begin{aligned}
\hat{u}(\mathbf{x}) & \equiv \frac{\hat{a}_{0}}{\hat{V}^{1 / 2}}+\tilde{\dot{u}}(\mathbf{x}) \\
\tilde{u}^{\dagger}(\mathbf{x}) & \equiv \frac{\hat{a}_{0}^{\dagger}}{\tilde{V}^{1 / 2}}+\tilde{u^{\dagger}}(\mathbf{x}) .
\end{aligned}
$$

Since the commutator of $\hat{a}_{0}$ and $\hat{a}_{0}^{\dagger}$ is unity, and is small compared with their product which is of order $V$. we can replace operators $\hat{a}_{0}$ and $\hat{a}_{0}^{\dagger}$ by the c-number.$_{0}^{1 / 2}$ in (2.2) and (2.23). This procedure, known as the Bogoliubor prescription. is appropriate when the number of particles in the zero-momentum state is a finite fraction of 1 . The error introduced is of the order $O\left(V^{-1}\right)$. and thus vanishes in the thermodynamic limit $(N \rightarrow \infty$ and $V \rightarrow \infty$, with fixed density $n=N / V)$. Clearly: the new field operators $\tilde{\psi}(\mathbf{x}), \tilde{\psi}^{\dagger}(\mathbf{x})$ describe the non-condensate atoms. and satisfy the Bose commutation relations $(2.2)$ in the thermodynamic limit. The average of $\therefore$ and $i t$ now vanishes as in the usual case of a normal system. namely

$$
\langle\tilde{\psi}(\mathbf{x})\rangle=\left\langle\tilde{\psi}^{\dagger}(\mathbf{x})\right\rangle=0
$$

With the above Bogoliubov broken-symmetry prescription, the thermal Green's 
function defined in (2.6) now separates into two parts:

$$
G\left(\mathbf{x} \tau \cdot \mathbf{x}^{\prime} \tau^{\prime}\right)=-n_{0}+\tilde{G}\left(\mathbf{x} \tau \cdot \mathbf{x}^{\prime} \tau^{\prime}\right)
$$

with the non-condensate Green s function defined by

$$
\tilde{G}\left(\mathbf{x} \tau \cdot \mathbf{x}^{\prime} \tau^{\prime}\right) \equiv-\left\langle T_{\tau} \tilde{\psi} \cdot(\mathbf{x} \tau) \tilde{\psi}^{\dagger}\left(\mathbf{x}^{\prime} \tau^{\prime}\right)\right\rangle
$$

In momentum representation for $\mathrm{k} \neq 0$. we have

$$
\tilde{G}(\mathbf{k} . \tau)=-\left\langle T_{r} \hat{a}_{\mathbf{k}}(\tau) \hat{a}_{\mathbf{k}}^{\dagger}(0)\right\rangle
$$

The separation of the condensate using (2.22) and (2.23) and the Bogoliubov prescription modify the Hamiltonian in a fundamental way. The grand canonical Hamiltonian is now given by

$$
\begin{aligned}
\hat{\Lambda} & =E_{0}-\mu \cdot V_{0}+\frac{1}{V} \sum_{\mathbf{k} \neq 0}\left(\epsilon_{k}-\mu\right) \hat{a}_{\mathbf{k}}^{\dagger} \hat{a}_{\mathbf{k}}+\sum_{j=1}^{i} \hat{l}_{j} \\
& \equiv \hat{h}_{0}+\sum_{j=1}^{j} \hat{V}_{j}
\end{aligned}
$$

where the interaction Hamiltonian $\hat{V}$ separates into eight distinct parts:

$$
\begin{aligned}
& E_{0}=\frac{1}{2} n_{0}^{2} V v(0) \\
& \hat{V_{1}}=\frac{1}{2} n_{0} \sum_{\mathbf{k}} v(\mathbf{k}) \hat{a}_{\mathbf{k}} \hat{a}_{-\mathbf{k}} \\
& \hat{V}_{2}=\frac{1}{2} n_{0} \sum_{\mathbf{k}} v(\mathbf{k}) \hat{a}_{\mathbf{k}}^{\dagger} \hat{a}_{-\mathbf{k}}^{\dagger} \\
& \hat{V}_{3}=n_{0} \sum_{\mathbf{k}} v(\mathbf{k}) \hat{a}_{\mathbf{k}}^{\dagger} \hat{a}_{\mathbf{k}} \\
& \hat{V}_{4}=n_{0} \sum_{\mathbf{k}} v(0) \hat{a}_{\mathbf{k}}^{\dagger} \hat{a}_{\mathbf{k}} \\
& \hat{V}_{5}=\frac{n_{0}^{1 / 2}}{V^{1 / 2}} \sum_{\mathbf{k} \cdot \mathbf{q}} v(\mathbf{q}) \hat{a}_{\mathbf{k}+\mathbf{q}}^{\dagger} \hat{a}_{\mathbf{k}} \hat{a}_{\mathbf{q}}
\end{aligned}
$$




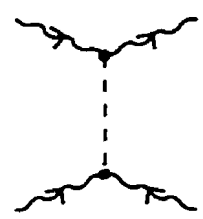

$\mathrm{E}_{\mathrm{o}}$

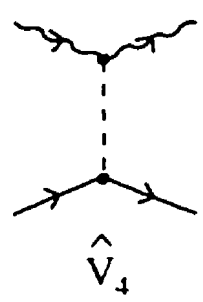

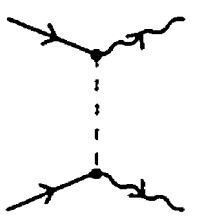

$\hat{\mathrm{V}}_{1}$

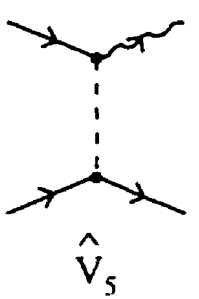

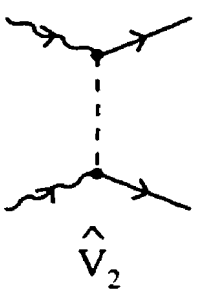

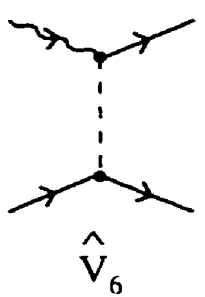

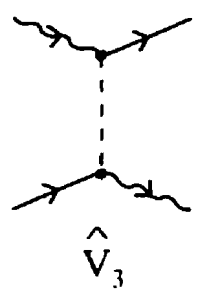

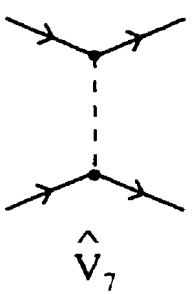

Figure 2.1: Eight distinct processes involved in the interaction $\dot{V}$. A solid line denotes $\hat{a}$ or $\hat{a}^{\dagger}$, a wiggly line corresponds to a condensate atom or a factor $n_{0}^{1 / 2}$. and a dashed line denotes the interaction $v$.

$$
\begin{aligned}
& \hat{l}_{6}=\frac{n_{0}^{1 / 2}}{\sqrt{1 / 2}} \sum_{\mathbf{k} \cdot \mathbf{q}} v(\mathbf{q}) \hat{a}_{\mathbf{k}}^{\dagger} \hat{a}_{\mathbf{q}}^{\dagger} \hat{a}_{\mathbf{k}+\mathbf{q}} \\
& \hat{l}_{\bar{c}}=\frac{1}{2 V} \sum_{\mathbf{k}, \mathbf{k}^{\prime} \cdot \mathbf{q}} v(\mathbf{q}) \hat{a}_{\mathbf{k}+\mathbf{q}}^{\dagger} \hat{a}_{\mathbf{k}^{\prime}-\mathbf{q}}^{\dagger} \hat{a}_{\mathbf{k}^{\prime}} \hat{a}_{\mathbf{k}}
\end{aligned}
$$

with

$$
v(\mathbf{q}) \equiv \int d \mathbf{x} e^{i \mathrm{q} \cdot \mathbf{x}} v(\mathbf{x})
$$

These parts are represented by the vertex diagrams in Fig.2.1. In a normal system $\left(n_{0}=0\right)$, only $\hat{V}_{i}$ is present. We also note that $\hat{K}$ has no term containing a single $\hat{a}_{\mathbf{k}}^{\dagger}$ or $\hat{a}_{\mathbf{k}}$ because these would violate momentum conservation. This is in agreement with (2.24), which gives in momentum representation

$$
\left\langle\hat{a}_{\mathbf{k}}\right\rangle=\left\langle\hat{a}_{\mathbf{k}}^{\dagger}\right\rangle=0 \quad(\mathbf{k} \neq 0)
$$




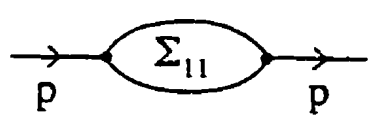

$\Sigma_{11}(\mathrm{p})$

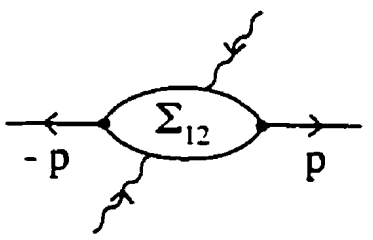

$\Sigma_{12}(\mathrm{p})$

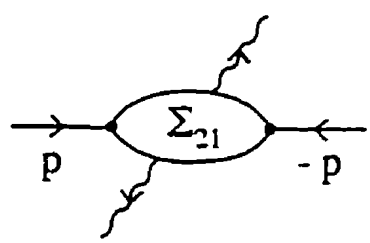

$\Sigma_{21}(\mathrm{p})$

Figure 2.2: Proper self-energies for a Bose-condensed system

\subsection{Dyson-Beliaev equations}

The fact that there exist interaction terms such as $V_{1}$ in Fig.2.1 implies the nonconservation of particles in the system described by non-condensate operators $\hat{a}_{\mathrm{k}}$ and $\hat{a}_{\mathbf{k}}^{\dagger}$, due to exchanges between non-condensate atoms and the condensate atoms. Verertheless, if a proper self-energy is defined as a part of a Feynman diagram connected to the rest of the diagram by two non-condensate particle lines. then it is still possible to analyze the contributions to the Green's function in a form similar to Dyson's equation for interacting fermions. However, there are now three distinct proper selfenergies, as indicated in Fig.2.2. One type of self-energy has one particle line going in and one coming out $\left(\Sigma_{11}\right)$, similar to that for fermions. The other ones have two particle lines either coming out $\left(\Sigma_{12}\right)$ or going in $\left(\Sigma_{21}\right)$, and reflect the new features associated with the existence of a Bose condensate reservoir. Correspondingly. we must also introduce two new Green`s functions.

$$
\begin{aligned}
& G_{12}(\mathbf{k}, \tau) \equiv-\left\langle T_{\tau} \hat{a}_{-\mathbf{k}}(\tau) \hat{a}_{\mathbf{k}}(0)\right\rangle, \\
& G_{21}(\mathbf{k}, \tau) \equiv-\left\langle T_{\tau} \hat{a}_{\mathbf{k}}^{\dagger}(\tau) \hat{a}_{-\mathbf{k}}^{\dagger}(0)\right\rangle .
\end{aligned}
$$

$G_{12}$ and $G_{21}$ are usually called the anomalous Green's functions, representing the disappearance and appearance of two non-condensate particles, respectively. The normal Green's function $\tilde{G}$ defined in (2.26) is denoted as $G_{11}$, representing the propagation of a single particle

$$
G_{11}(\mathbf{k}, \tau) \equiv-\left\langle T_{\tau} \hat{a}_{\mathbf{k}}(\tau) \hat{a}_{\mathbf{k}}^{\dagger}(0)\right\rangle
$$




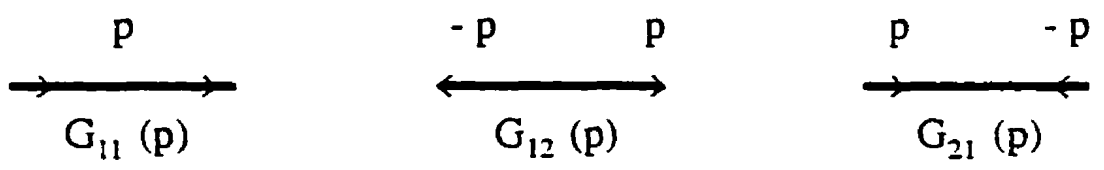

Figure 2.3: Green's functions for a Bose-condensed system.

These three Green's functions are shown in Fig.2.3. where the arrows indicate the direction of momentum of the atoms involved.

The Dyson equations for this system were first derived by Beliaev [11], which in frequency-momentum space are given by:

$$
\begin{aligned}
& G_{11}(p)=G^{(0)}(p)+G^{(0)}(p) \Sigma_{11}(p) G_{11}(p)+G^{(0)} \Sigma_{21}(p) G_{21}(p) \\
& G_{12}(p)=G^{(0)}(p) \Sigma_{12}(p) G_{11}(-p)+G^{(0)}(p) \Sigma_{11}(p) G_{12}(p) . \\
& G_{21}(p)=G^{(0)}(-p) \Sigma_{21}(p) G_{11}(p)+G^{(0)}(-p) \Sigma_{11}(-p) G_{21}(p) .
\end{aligned}
$$

For simplicity. we use the letter $p$ to represent the four-dimensional vector $\left(k . i \omega_{n}\right)$. Unless otherwise noted. we follow this convention in the rest of the thesis. The DysonBeliaev equations (2.43)-(2.4.5) are illustrated diagrammatically in Fig.2.4. The structure of these equations can be simplified by introducing a matrix operator [38]

$$
\hat{\mathbf{A}}_{\mathbf{k}} \equiv\left[\begin{array}{l}
\hat{a}_{\mathbf{k}} \\
\hat{a}_{\mathrm{k}}^{\dagger}
\end{array}\right] \text {. }
$$

and. correspondingly, a $2 \times 2$ matrix Green`s function

$$
\mathbf{G}(\mathbf{k}, \tau) \equiv-\left\langle T_{\tau} \hat{\mathbf{A}}_{\mathbf{k}}(\tau) \hat{\mathbf{A}}_{\mathbf{k}}^{\dagger}(0)\right\rangle
$$

A single matrix equation can represent the three Dyson's equations of (2.43)-(2.45). namely

$$
\mathbf{G}(p)=\mathbf{G}^{(0)}(p)+\mathbf{G}^{(0)}(p) \Sigma(p) \mathbf{G}(p),
$$



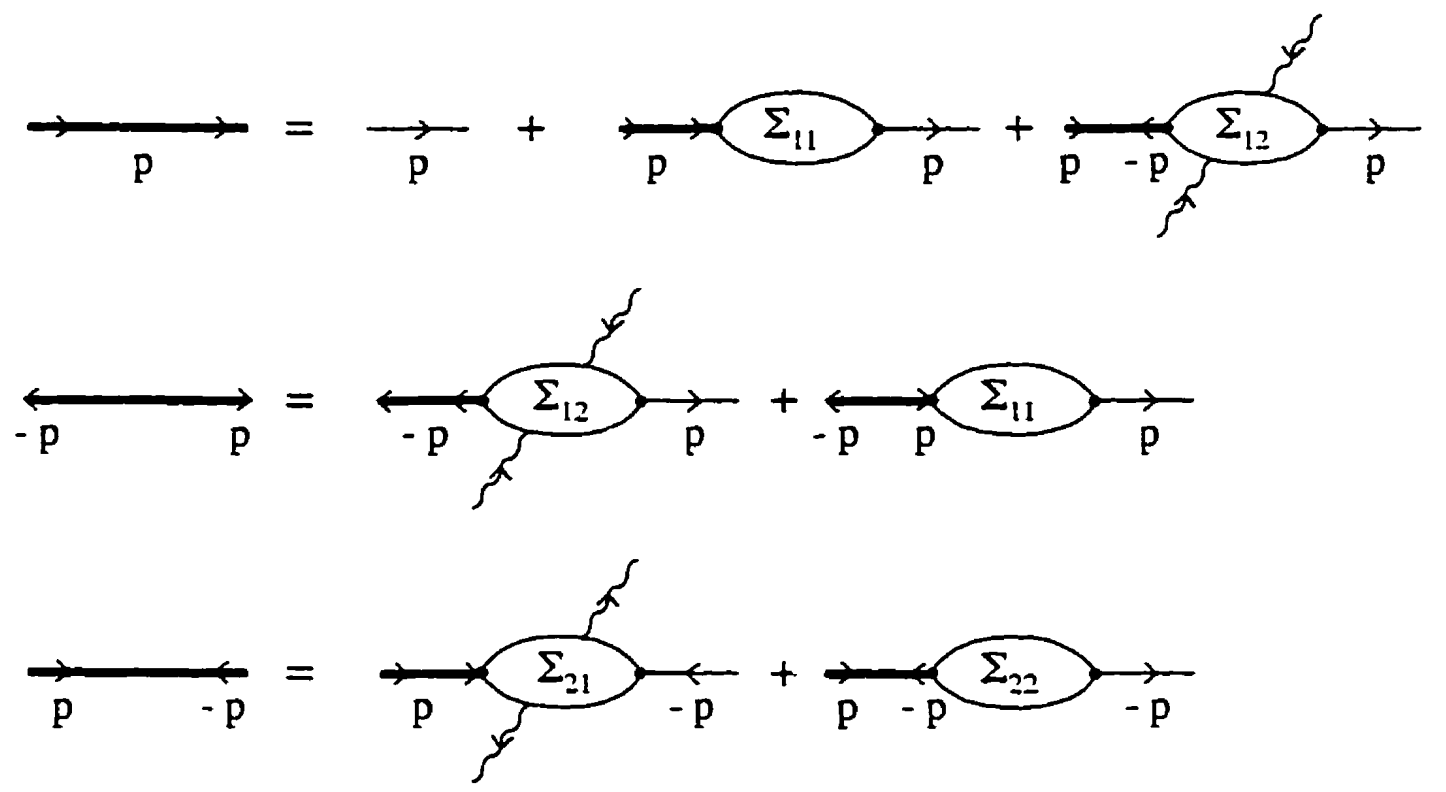

Figure 2.4: Dyson-Beliaev equations for a Bose-condensed system.

with

$$
\begin{aligned}
\mathbf{G}(p) & =\left[\begin{array}{ll}
G_{11}(p) & G_{12}(p) \\
G_{21}(p) & G_{22}(p)
\end{array}\right] . \\
\mathbf{G}^{(0)}(p) & =\left[\begin{array}{cc}
G^{(0)}(p) & 0 \\
0 & G^{(0)}(-p)
\end{array}\right] . \\
\Sigma(p) & =\left[\begin{array}{ll}
\Sigma_{11}(p) & \Sigma_{12}(p) \\
\Sigma_{21}(p) & \Sigma_{22}(p)
\end{array}\right] .
\end{aligned}
$$

Here the unperturbed Green's function is given by

$$
G^{(0)}(p) \equiv G^{(0)}\left(\mathbf{k}, i \omega_{n}\right)=\frac{1}{i \omega_{n}-\epsilon_{k}+\mu}
$$

The matrix elements are not independent, with the following useful identities:

$$
\begin{array}{ll}
G_{22}(p)=G_{11}(-p), & G_{12}(p)=G_{21}(-p) \\
\Sigma_{22}(p)=\Sigma_{11}(-p), & \Sigma_{12}(p)=\Sigma_{21}(-p) .
\end{array}
$$


Eq.(2.48) can be solved to give

$$
\begin{aligned}
& G_{11}(p)=\frac{i \omega_{n}+\epsilon_{k}-\mu+\Sigma_{11}(-p)}{\left[i \omega_{n}+\epsilon_{k}-\mu+\Sigma_{11}(-p)\right]\left[i \omega_{n}-\epsilon_{k}+\mu-\Sigma_{11}(p)\right]+\left[\Sigma_{12}(p)\right]^{2}} . \\
& G_{12}(p)=\frac{-\Sigma_{12}(p)}{\left[i \omega_{n}+\epsilon_{k}-\mu+\Sigma_{11}(-p)\right]\left[i \omega_{n}-\epsilon_{k}+\mu-\Sigma_{11}(p)\right]+\left[\Sigma_{12}(p)\right]^{2}} .
\end{aligned}
$$

These last two equations express the normal and anomalous Green's functions in terms of the exact proper self-energies. and therefore are entirely general. They apply to any uniform Bose-condensed fluid. liquid or gas.

We see that in (2.53) and (2.54). both Green's functions share the same poles. Moreover it is easy to check that in the $\mathrm{k} \rightarrow 0$ limit. this pole occurs at $i \omega_{n}=0$ if

$$
\mu=\Sigma_{11}(0.0)-\Sigma_{12}(0.0)
$$

In fact. (2.55) can be shown to be true to all orders in perturbation theory and is known as the Hugenholtz-Pines theorem [2:3]. It was first derived by Hugenholtz and Pines for the $T=0$ case by a direct diagrammatic analysis. and generalized to finite temperature by Hohenberg and Martin [26]. This theorem is very important because it shows. quite generally, the energy $E(\mathbf{k})$ of single particle excitations (which are related to the poles of $C_{\alpha 3}$ ) vanishes at $\mathrm{k}=0$ : in other words. the excitations have no energy gap in the long wavelength limit. The Hugenholtz-Pine theorem thus provides a criterion for ensuring such a gapless approximation [26].

\subsection{Real-time Green's functions}

In Section 2.2. we pointed out that the thermal Green's functions $G$ can be used to calculate thermodynamic properties. Through its relation to the real-time Gireen's function. it also can be used to determine the energy and lifetime of the states of a system when one adds or subtracts a single atom.

As usual, we restrict ourselves to the case of a homogeneous system with a timeindependent Hamiltonian. Then the eigenstates $|m\rangle$ of the grand canonical Hamilto- 
nian $\hat{K}$ and the momentum operator $\hat{\mathbf{P}}$ are the same, with

$$
\begin{aligned}
& \hat{\kappa}|m\rangle=E_{m}|m\rangle . \\
& \hat{\mathbf{P}}|m\rangle=\mathbf{P}_{m}|m\rangle .
\end{aligned}
$$

In such a basis. the imaginary-frequency Green's function $G\left(\mathbf{k}\right.$. $\left.i \omega_{n}\right)$ can be written in a Lehmann representation (see, for example. Chapter 9 of [1:3]), which yields the following exact spectral representation

$$
C_{(}\left(\mathbf{k} \cdot i \omega_{n}\right)=\int_{-\infty}^{\infty} \frac{d \omega^{\prime}}{2 \pi} \frac{A\left(\mathbf{k} \cdot \omega^{\prime}\right)}{i \omega_{n}-\omega^{\prime}}
$$

where $A(k \cdot u)$ is the single-particle spectral density function defined by

$$
\begin{aligned}
A(\mathbf{k} \cdot \omega) \equiv \epsilon^{3 \Omega} \sum_{m n}\left\{e^{-3 E_{m}(2 \pi)^{3} \delta\left[\mathbf{k}-\hbar^{-1}\left(\mathbf{P}_{n}-\mathbf{P}_{m}\right)\right]}\right. \\
\left.\times 2 \pi \delta\left[\omega-\left(E_{n}-E_{m}\right)\right]\left(1-\epsilon^{-3 \omega}\right)|\langle m|\dot{\omega}(\mathbf{x}=0)| n\rangle|^{2}\right\}
\end{aligned}
$$

Following directly from its definition. one can show quite generally that for a Bose system. $f(\mathbf{k} . w)$ has the following properties (see. for example. Chapter 9 of [1:3]):

$$
\begin{aligned}
& \operatorname{sgn}(\omega) A(\mathbf{k} \cdot \omega) \leq 0 . \\
& \int_{-\infty}^{\infty} \frac{d \omega}{2 \pi} A(\mathbf{k} \cdot \omega)=1 . \\
& \int_{-\infty}^{\infty} \frac{d \omega}{2 \pi} f_{B}(\omega) \cdot A(\mathbf{k}, \omega)=n_{\mathbf{k}} .
\end{aligned}
$$

where $f_{B}(\omega)$ is the Bose distribution function and $n_{k}$ is the momentum distribution of atoms. In (2.60), the equality holds if $\omega=0$. Equation (2.61) is an example of a frequency-moment sum rule which is useful as a check on specific approximations for $A(\mathbf{k}, \omega)$. For other frequency-moment sum rule see Chapter $\delta$ of [ 8$]$. The spectral density function $A(\mathbf{k}, \omega)$ and its relation to $C_{\boldsymbol{r}}$ in $(2.58)$ plays a central role in the finitetemperature formalism. since one can show that the same $A(\mathbf{k}, \omega)$ also determines the various real-time Green's functions. 
At finite temperature, the retarded and advanced Green's functions involving real time $t$ are defined as

$$
\begin{aligned}
G_{r}^{\mathrm{ret}}\left(\mathbf{x} t \cdot \mathbf{x}^{\prime} t^{\prime}\right) & \equiv-i \theta\left(t-t^{\prime}\right)\left\langle\left[\hat{\psi}(\mathbf{x} t) \hat{\psi^{\dagger}}\left(\mathbf{x}^{\prime} t^{\prime}\right)-\hat{\psi}^{\dagger}\left(\mathbf{x}^{\prime} t^{\prime}\right) \hat{\psi}(\mathbf{x} t)\right]\right\rangle \\
G_{r}^{\mathrm{adv}}\left(\mathbf{x} t \cdot \mathbf{x}^{\prime} t^{\prime}\right) & \equiv-i \theta\left(t^{\prime}-t\right)\left\langle\left[\hat{\psi}(\mathbf{x} t) \hat{\psi^{\dagger}}\left(\mathbf{x}^{\prime} t^{\prime}\right)-\hat{\psi}^{\dagger}\left(\mathbf{x}^{\prime} t^{\prime}\right) \hat{\iota^{\prime}}(\mathbf{x} t)\right]\right\rangle
\end{aligned}
$$

Here $\langle\cdots\rangle$ denotes the grand canonical ensemble average: and the real time Heisenberg operator $\dot{\psi}(\mathrm{x} t)$ are defined by

$$
\hat{\dot{U}}(\mathbf{x} t)=\epsilon^{i \hat{K} t / \hbar} \dot{\psi} \cdot(\mathbf{x}) \epsilon^{-i \hat{K} t / \hbar}
$$

$C_{r}^{\mathrm{ret}}$ and $G^{\mathrm{adv}}$ may be also written in the Lehmann representation. and expressed in terms of the spectral density function $A(k . \omega)$ defined in (2.59) as [1:3]

$$
\begin{aligned}
C^{\mathrm{ret}}\left(\mathbf{k} \cdot \omega^{\prime}\right) & =\int_{-\infty}^{\infty} \frac{d \omega^{\prime}}{2 \pi} \frac{A\left(\mathbf{k} \cdot \omega^{\prime}\right)}{\omega-\omega^{\prime}+i \eta} . \\
G^{\mathrm{adv}}\left(\mathbf{k} \cdot \omega^{\prime}\right) & =\int_{-\infty}^{x} \frac{d \omega^{\prime}}{2 \pi} \frac{A\left(\mathbf{k} \cdot \omega^{\prime}\right)}{\omega-\omega^{\prime}-i \eta} .
\end{aligned}
$$

Equation (2.58). (2.66) and (2.67) show that the function $A(k . u)$ determines the thermal Green's function as well as $C_{r}^{\text {ret }}$ and $C_{r}^{\text {radv }}$. suggesting the following analytic continuation:

$$
\begin{aligned}
& C\left(\mathbf{k} \cdot i \omega_{n} \rightarrow \omega+i \eta\right)=G^{\mathrm{ret}}(\mathrm{k} \cdot \omega) . \\
& G\left(\mathbf{k}, i \omega_{n} \rightarrow \omega-i \eta\right)=G^{\mathrm{adv}}(\mathbf{k} \cdot \omega) .
\end{aligned}
$$

Although $G\left(\mathbf{k}, i \omega_{n}\right)$ is a function only at the discrete set of points iwn along the imaginary frequency axis, it can give $G^{\text {ret }}$ and $G^{\text {adv }}$ through the analytic continuation to the real axis by using (2.68) and (2.69). Since the sum rule (2.61) requires that both $C_{r}^{\text {ret }}($ k. $\omega)$ and $C_{r}^{\text {adv }}(\mathbf{k}, \omega) \sim \omega^{-1}$ as $|\omega| \rightarrow \infty$, the above analytic continuation is guaranteed to be unique [39]. Using these results, $A(\mathbf{k}, \omega)$ can be also written as

$$
A(\mathrm{k}, \omega)=-2 \operatorname{Im} G_{r}^{\mathrm{ret}}(\mathbf{k}, \omega)
$$




$$
=-2 \operatorname{Im} G\left(\mathbf{k}, i \omega_{n} \rightarrow \omega+i \eta\right) .
$$

Therefore. any approximation for the thermal Green's function immediately yields a corresponding $A\left(k, \omega^{\prime}\right)$, as well as an approximation for $G_{r}^{\text {ret }}$ and $G^{\text {adv }}$.

For completeness. we discuss the relation between the thermal Green's function and the real-time Green's function used at $T=0$. For this purpose. we define the following real-time Green's function at finite temperature. in direct analogy to that at $T=0$ :

$$
\bar{C}\left(\mathbf{x} t \cdot \mathbf{x}^{\prime} t^{\prime}\right) \equiv(-i) \operatorname{Tr}\left\{e^{3(\Omega-\hat{K})} T_{t}\left[\hat{\dot{\psi}}(\mathbf{x} t) \hat{i}^{\dagger}\left(\mathbf{x}^{\prime} t^{\prime}\right)\right]\right\}
$$

Here $T_{t}$ is the real-time order operator. Again. by means of a Lehmann representation. the Fourier transform of $\bar{G}$ can be shown to be related to $C_{r}^{\text {ret }}$ and $G_{r}^{\text {adv }}$ by [1:3]

$$
\begin{aligned}
\bar{G}(\mathbf{k}, \omega) & \equiv \int_{-\infty}^{\infty} d t e^{i \omega t} \bar{G}(\mathbf{k} \cdot t) \\
& =\left[1+f_{B}(\omega)\right] G^{\mathrm{ret}}(\mathbf{k} \cdot \omega)-f_{B}(\omega) G^{\mathrm{rdv}}(\mathbf{k} \cdot \omega)
\end{aligned}
$$

In the limit of $T \rightarrow 0$, the last equation reduces to

$$
\begin{aligned}
\bar{C}^{T=0}(\mathbf{k} \cdot \omega) & =\theta(\omega) G^{\mathrm{ret}}(\mathbf{k} \cdot \omega)+\theta(-\omega) C^{\mathrm{adv}}(\mathbf{k} \cdot \omega) \\
& = \begin{cases}G\left(\mathrm{k} \cdot i \omega_{n} \rightarrow \omega+i \eta\right) & \omega>0 \\
G\left(\mathbf{k}, i \omega_{n} \rightarrow \omega-i \eta\right) & \omega<0\end{cases}
\end{aligned}
$$

Eq.(2.73) shows explicitly how one obtains the $T=0$ Green's function from the imaginary frequency thermal Green's function using the analytical continuation.

\subsection{The Bogoliubov approximation}

To illustrate the formalism we have briefly reviewed in this chapter, we use it to solve for $G_{11}$ and $G_{12}$ in the famous Bogoliubov approximation [9].

One expects that in a weakly-interacting Bose gas at low temperatures. most of the atoms will still remain in the $\mathbf{k}=0$ state, only a few of them being "kicked" out of the condensate by the interactions. The number of non-condensate atoms 

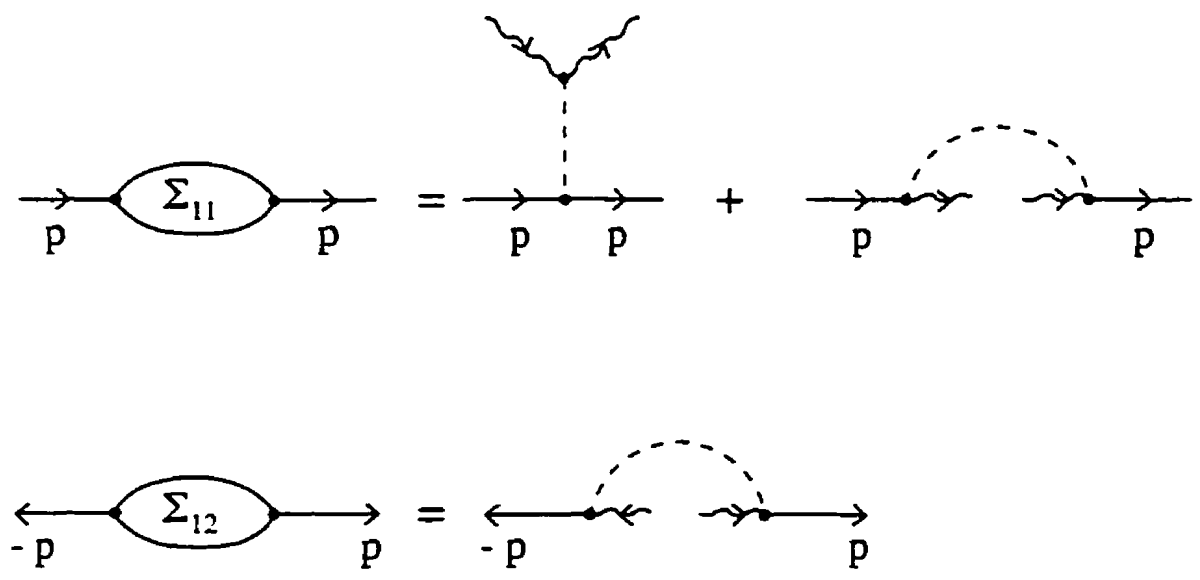

Figure 2.5: Self-energy diagrams in the Bogoliubor approximation.

is usually called the "depletion". If the depletion is small. the interaction between two non-condensate atoms will not be important. This is the basic assumption that leads to the so-called Bogoliubov model. in which the terms $\hat{V}_{5} . \hat{V}_{6}$ and $\hat{V}_{7}$ in $(2.29)$ are neglected. The remaining terms are quadratic. diagonalizable by a canonical transformation (see. for example. Chapter 25 of [36]). This Bogoliubov model can be described in terms of Green`s function language. as first discussed by Beliaev [11]. In Green's function language. the Bogoliubov approximation consists of keeping the lowest-order self-energy diagrams that contain condensate lines, as shown in Fig.2.5. These can be written as

$$
\begin{aligned}
& \Sigma_{11}(p)=n_{0}[v(0)+v(\mathbf{k})], \\
& \Sigma_{12}(p)=n_{0} v(\mathbf{k}),
\end{aligned}
$$

where $n_{0} \equiv N_{0} / V$ is called the condensate density. An important feature of these selfenergies is that they are frequency independent. With the Hugenholtz-Pines theorem [23], the lowest-order contribution to the chemical potential is given by

$$
\mu=n_{0} v(0) .
$$


Lsing these results in (2.53) and (2.54). we obtain

$$
\begin{aligned}
& G_{11}(p)=\frac{i \omega_{n}+\epsilon_{k}+n_{0} v(\mathbf{k})}{\left(i \omega_{n}\right)^{2}-\epsilon_{k}^{2}-2 n_{0} v(\mathbf{k}) \epsilon_{k}} . \\
& G_{12}(p)=\frac{-n_{0} v(\mathbf{k})}{\left(i \omega_{n}\right)^{2}-\epsilon_{k}^{2}-2 n_{0} v(\mathbf{k}) \epsilon_{k}} .
\end{aligned}
$$

One most striking feature of the Bogoliubov model is the form of the excitation spectrum $E_{\mathbf{k}}$. given by the poles of $C_{r_{11}}$ and $C_{12}$.

$$
E_{\mathbf{k}}=\sqrt{\epsilon_{k}^{2}+2 n_{0} v(\mathbf{k}) \epsilon_{k}}
$$

which agrees with the result from a direct canonical diagonalization. In the long wavelength limit $(\mathbf{k} \rightarrow 0), E_{\mathbf{k}}$ reduces to the phonon-like dispersion relation

$$
E_{\mathrm{k}} \sim h c k \quad|\mathbf{k}| \rightarrow 0
$$

with the characteristic velocity

$$
c=\left[\frac{n_{0} v(0)}{m}\right]^{1 / 2}
$$

A detailed calculation shows that the ground-state energv ( $\sec \operatorname{section} 2 \cdot 2$ of $[1: 3]$ ) is given by

$$
E_{0}=\frac{1}{2} \frac{V^{2}}{V} v(0)
$$

which yields the pressure

$$
P=-\left(\frac{\partial E_{0}}{\partial V}\right)_{N}=\frac{1}{2} n^{2} v(0)
$$

and hence the macroscopic speed of sound

$$
s \equiv\left[\frac{1}{m} \frac{\partial P}{\partial n}\right]^{1 / 2}=\left[\frac{n v(0)}{m}\right]^{1 / 2}
$$

Comparison of (2.81) and (2.84) shows that $c$ equals the macroscopic speed of sound 
$s$ given by the usual thermodynamic derivative. Indeed. it has been proved to all orders in perturbation theory $[22,26]$ for the $T=0$ case that the single-particle excitation spectrum vanishes linearly as $|\mathbf{k}| \rightarrow 0$. with a slope equal to the macroscopic speed of sound. This linear dependence of excitation spectrum at long wavelengths is the essential piece of physics that already emerges from the simple Bogoliubov approximation. At low temperatures, the phonon spectrum will make the dominant contribution to thermodynamic properties and thus the Bogoliubov results gives the low-temperature behavior characteristic of Bose-condensed fluids.

In this chapter. we have given a brief review of the finite-temperature Green's function formalism for a Bose-condensed system. Due to the macroscopic occupation of the lowest energy state, one must introduce the anomalous (ireen's functions $C_{12}$ and $C_{21}$ in addition to the normal Green's function $C_{11}$, and correspondingly. the selfenergies $\Sigma_{12} . S_{21}$ and $\Sigma_{11}$. Approximate solutions are determined by our choice of selfenergies. In the succeeding chapters. we discuss finite temperature approximations for a Bose condensed system. namely. the first-order Popov approximation and the second-order Beliaev-Popov approximation (in which the self-energies are explicitly first order and second order in the interaction. respectively). 


\section{Chapter 3}

\section{The first-order Popov approximation}

In this chapter. we discuss a first-order self-energy approximation for a weaklyinteracting Bose gas at finite temperatures. It was first discussed in 1965 by Popor [32]. and thus we call it the Popor approximation [1.5]. The well-known Bogoliubov approximation discussed in Section 2.5 only describes the physics of a lowtemperature Bose gas. Clearly, as the number $\dot{V}$ of excited atoms (those not in the zero-momentum state) increases with temperature. the Bogoliubov approximation becomes inappropriate since it assumes $. \dot{V}=. V-. V_{0} \ll . V$ and consequently ignores the effect of interactions between the excited atoms. At finite temperature when $\dot{V}$ is not small compared to $N$. the interactions between the excited atoms must be taken into account. This is done in the Popov approximation by treating the excited atoms in the Hartree-Fock approximation. Apart from Popov's work, most theories of temperature-dependent properties (for example. Ref.[40]) of a weakly-interacting Bose gas have been based on the Bogoliubov model. which means they are limited to the low temperature $\left(T \ll T_{c}\right)$ region. The Popov approximation. as noted in Refs. $[15,41]$, gives a reasonable first approximation for a weakly-interacting Bose gas at much higher temperatures.

In the present chapter, we will give explicit expressions of the self-energies in the Popov approximation, and calculate the Green's functions and some physical 
quantities at finite temperatures. We will also discuss the reason why the Popor approximation is not valid in the very small temperature interval near $T_{c}$.

$$
\left|T-T_{c}\right| \lesssim\left(n^{1 / 3} a\right) T_{\varepsilon}
$$

The Popov approximation was largely unknown until very recently. when it was first applied to discuss the BEC of paraexcitons in $\mathrm{Cu}_{2} \mathrm{O}$ by Shi. Verechaka and Griffin [1.5]. In this work, a general expression for decay luminescence spectrum from a gas of excitons was derived. This was evaluated at finite temperatures using the Popor approximation as a simple first-order theory of the effects of interaction in a dilute weakly-interacting gas of excitons.

\subsection{The HFB self-energies}

In his original paper. Popov [32] expressed the self-energies in terms of the $t$-matrix (more precisely, the ladder approximation of the $t$-matrix). instead of the bare potential $v(\mathbf{r})$. The $t$-matrix is a more effective description of the effective interactions between the atoms than the interatomic potential itself. However. in order to emphasize the physics instead of going into the details of many-body scattering theory. we shall first proceed assuming that the bare potential $v(\mathbf{r})$ and its Fourier component $v(\mathrm{k})$ is weak and well-defined. After we have discussed the basic physics of the Popor approximation. we will incorporate the t-matrix into our results.

The self-energy diagrams in the Popov approximation include all possible firstorder diagrams of a Bose-condensed system. Among the diagrams in Fig.3.1. only those (a. b. e) containing two condensate lines are included in the Bogoliubov approximation of Section 2.5. The new ones (c and d) are the Hartree-Fock diagrams including excited atoms. The self-energy $\Sigma_{11}$ and $\Sigma_{12}$ of Fig.3.1 can be explicitly written as

$$
\Sigma_{11}(\mathbf{k})=n_{0}[v(0)+v(\mathbf{k})]-\frac{1}{3 V} \sum_{\mathbf{q}, i \omega_{n}} G^{(0)}\left(\mathbf{q}, i \omega_{n}\right)[v(0)+v(\mathbf{k}-\mathbf{q})]
$$




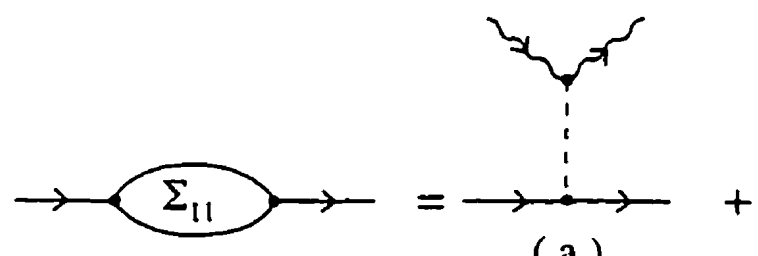

(a)
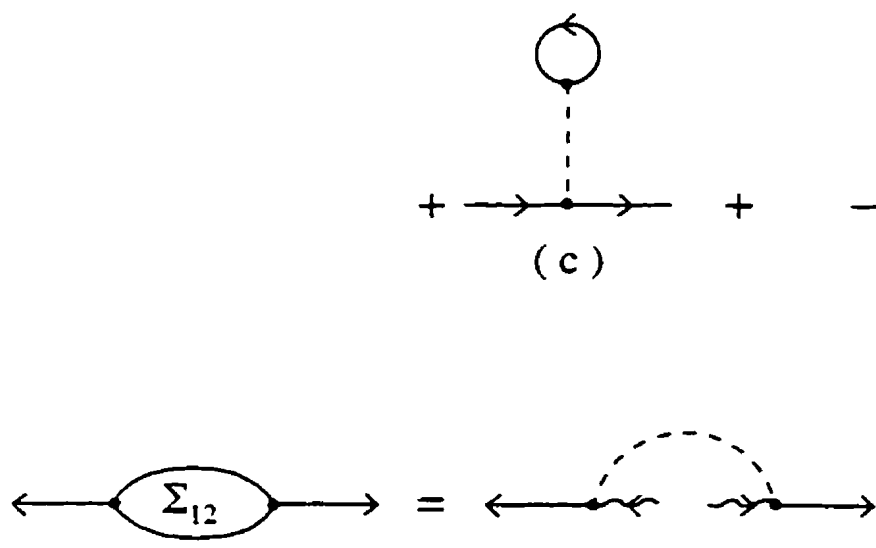

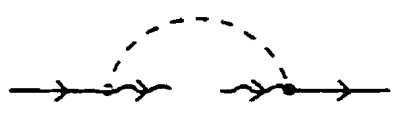

(b)

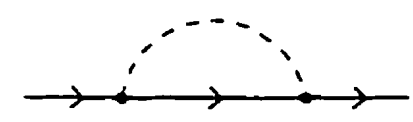

(d)

(e)

Figure 3.1: Self-energy diagrams in the Popov approximation involving the bare potential $v$. A solid line with arrow denotes an ideal gas propagator $G^{(0)}$. a wiggly line denotes a condensate atom. and a dash line denotes the interaction $v$

$$
\Sigma_{12}(\mathbf{k})=n_{0} v(\mathbf{k})
$$

Here $G^{(0)}\left(\mathbf{q}_{1} i \omega_{l}\right)$ is the unperturbed Green's function of non-interacting atoms, given by

$$
G^{(0)}\left(\mathbf{q}, i \omega_{l}\right)=\frac{1}{i \omega_{l}-\epsilon_{q}+\mu^{(0)}} .
$$

with $\mu^{(0)}$ denoting the chemical potential of an ideal Bose gas.

Since $v(\mathbf{k})$ does not depend on the Matsubara frequency $i \omega_{n}$, one sees that neither do $\Sigma_{11}(\mathrm{k})$ and $\Sigma_{12}(\mathrm{k})$. One can then perform the frequency sum in (3.2) to obtain

$$
\begin{aligned}
& \Sigma_{11}(\mathbf{k})=n_{0}[v(0)+v(\mathbf{k})]+\int \frac{d \mathbf{q}}{(2 \pi)^{3}} \frac{v(0)+v(\mathbf{k}-\mathbf{q})}{e^{\beta\left(\epsilon_{q}-\mu^{(0)}\right)}-1} . \\
& \Sigma_{12}(\mathbf{k})=n_{0} v(\mathbf{k}) .
\end{aligned}
$$

For $T \rightarrow 0$, the Bose distribution function $\left[\exp \beta\left(\epsilon_{q}-\mu^{(0)}\right)-1\right]^{-1}$ becomes very small, and thus the contribution due to interactions between two excited atoms (the second 
term in $\Sigma_{11}$ ) is negligible compared to that due to interactions between one excited atom and one condensate atom (the first term in $\Sigma_{11}$ ). In this limit. the Popor approximation reduces to the Bogoliubov approximation [see (2.it) and (2.75)]. as expected.

On the other hand. the condensate density $n_{0}(T)$ vanishes when $T>T_{\varepsilon}$ (normal phase). In this case, we are only left with diagrams (c) and (d) in Fig.3.1. i.t. the usual Hartree [(c)] and Fock [(d)] diagrams of a normal system. The Hartree-Fock approximation is basically a static mean-field theory. which treats the motion of single particles in an average static field generated by all the other particles. In the Popov approximation. the average Hartree-Fock field is determined by non-interacting particles as represented by the ideal gas propagator $C^{(0)}$ in diagrams (c) and (d). Taking into account the fact that the background particles also move in the average field coming from the presence of all the other particles. one can improve the HartreeFock theory by replacing the free propagator $G^{(0)}$ by the full renormalized propagator C. This is the well-known self-consistent Hartrec-Fock (SCHF) approximation [13. $39]$.

For a Bose-condensed system. the analogue of this self-consistent approach is called the self-consistent Hartree-Fock-Bogoliubov (HFB) approximation (for further discussion and earlier references. see Ref. $[41])$. In this self-consistent HFB approximation. one replaces the free propagator $G^{(0)}$ in Fig.3.1 by the matrix propagator G given by (2.4 $\tau$ ). which includes both the diagonal (normal) Gireen s functions ( $G_{11}$ and $\left(i_{22}\right)$ and the off-diagonal (anomalous) Green's functions $\left(C_{12}\right.$ and $\left.C_{r_{21}}\right)$. The self-consistent HFB approximation. however. has well-known problens in the Bosecondensed phase. such as the violation of various conservation laws and the presence of an energy gap in the excitation spectrum[26, 41]. As Griffin [41. 42] has pointed out, these problems with the self-consistent HFB approximation arise because the condensate atoms and excited atoms are not treated in an equal manner: the condensate atoms are treated in a collective way while the excited atoms are only treated as renormalized single particles. The Popov approximation corresponds to a simplified version of the HFB which does not introduce an energy gap in the excitation 


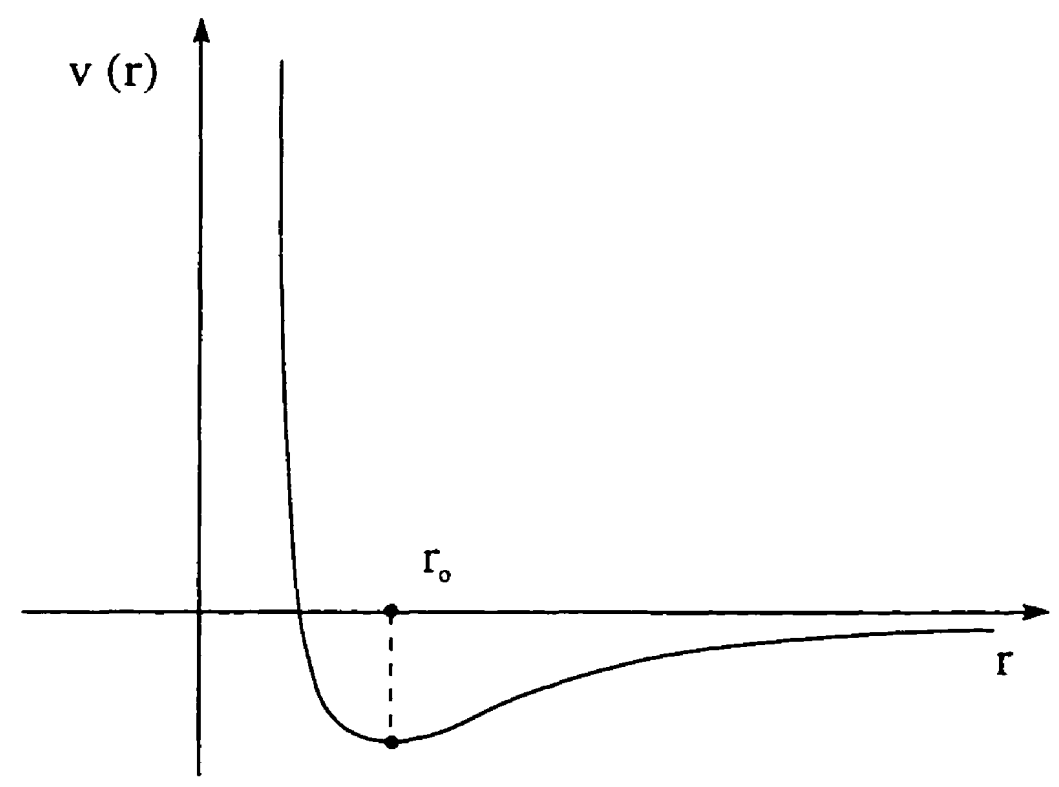

Figure 3.2: Typical inter-atomic potential

spectrum. as will be discussed shortly.

With the self-energies given by (3.5) and (3.6), one can. in principle. calculate the single-particle properties related to single-particle Green`s function $C_{\alpha, 3}$ for a given interparticle potential $v(\mathbf{r})$, assuming the interparticle potential has a well-defined Fourier transform $v(\mathbf{k})$. However the typical inter-atomic potential has a weak longrange attractive tail and a strong short-range repulsive core. as sketched in Fig.3.2. The hard core means that the Fourier transform $v(\mathbf{k})$ in $(3.5)$ and $(3.6)$ is not welldefined; indeed. it is singular. We now discuss how this problem can be solved.

\subsection{The Popov self-energies in terms of the $t$ - matrix}

Fortunately, for a low-density or dilute gas of atoms, one can make use of the ladder approximation. The word "dilute" has the meaning that the average interatomic distance, $d \sim n^{-1 / 3}$, is much larger than the s-wave scattering length $a$. which is the characteristic length representing the influence of the interatomic potential (see Appendix $\mathrm{A}$, where the basic elements of scattering theory are reviewed). This condition 


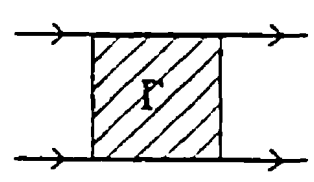

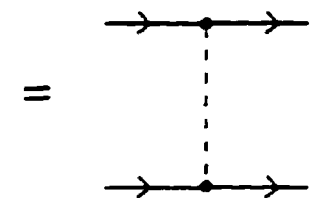

Lowest order

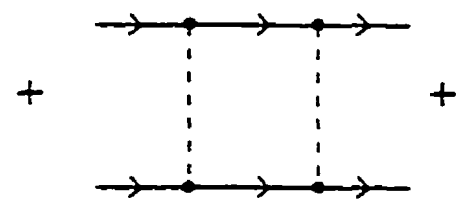

Second order
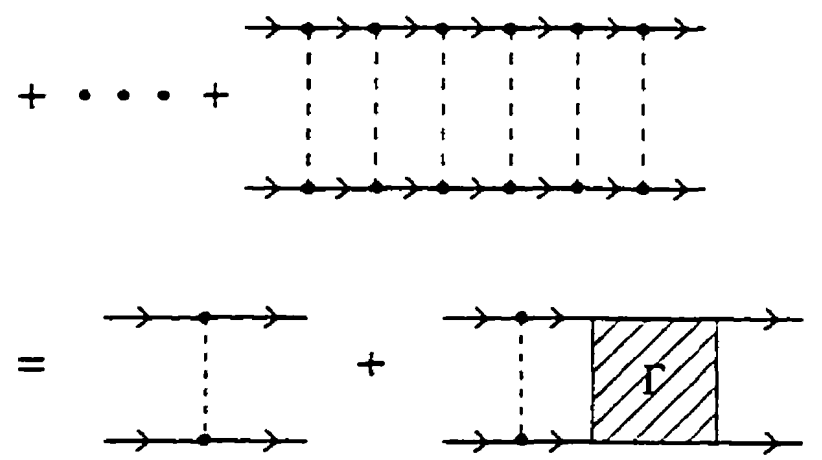

Figure 3.3: Diagrammatic definition of the $t$-matrix $\Gamma$

inplies that

$$
a / d \sim n^{1 / 3} a \ll 1 .
$$

As discussed by Beliaev [34], in a dilute gas, the ladder diagrams are all of equal importance. This sum over all the ladder diagrams yields the so-called many-body $t$ matrix $\Gamma$, as illustrated by the hatched square in Fig.3.3. The diagrammatic definition of $\Gamma$ in Fig.3.3 can be written explicitly as

$$
\begin{aligned}
\Gamma\left(p_{1}, p_{2} ; p_{3}, p_{4}\right) \equiv v & \left(\mathbf{k}_{1}-\mathbf{k}_{3}\right)-\frac{1}{\beta V} \sum_{\mathbf{q}, i \omega_{l}} v(\mathbf{q}) G^{(0)}\left(\mathbf{k}_{1}-\mathbf{q}, i \omega_{1}-i \omega_{l}\right) \\
& \times G^{(0)}\left(\mathbf{k}_{2}+\mathbf{q}, i \omega_{2}+i \omega_{l}\right) \Gamma\left(p_{1}-q, p_{2}+q: p_{3}, p_{4}\right),
\end{aligned}
$$

where the four-dimensional vector $p_{j} \equiv\left(\mathbf{k}_{j}, i \omega_{j}\right)$ represents the momentum $\mathbf{k}_{j}$ and Matsubara frequency $i \omega_{j}$ of a particle before $(j=1,2)$ or after $(j=3, t)$ scattering; similarly, $q \equiv\left(q, i \omega_{l}\right)$. It is often more convenient to write $\Gamma$ in the center-of- 
momentum frame of the scattering pair of atoms (see Appendix $A$ )

$$
\begin{aligned}
& \Gamma\left(\mathbf{k}, \mathbf{k}^{\prime}, \mathbf{K}: z\right) \equiv \Gamma\left(p_{\mathbf{l}}, p_{2}: p_{3} \cdot p_{4}\right) \\
& \quad=\iota\left(\mathbf{k}-\mathbf{k}^{\prime}\right)+\int \frac{d \mathbf{q}}{(2 \pi)^{3}} v(\mathbf{q}) \frac{F_{+}(\mathbf{K} \cdot \mathbf{k}-\mathbf{q})}{z-2\left(\epsilon_{\mathbf{k}}-\mathbf{q}-\mu\right)} \Gamma\left(\mathbf{k}-\mathbf{q} \cdot \mathbf{k}^{\prime} \cdot \mathbf{K}: z\right) .
\end{aligned}
$$

where we have introduced the center-of-mass variables

$$
\begin{aligned}
& \mathrm{k} \equiv \frac{\mathrm{k}_{1}-\mathrm{k}_{2}}{2} . \quad \mathrm{k}^{\prime} \equiv \frac{\mathrm{k}_{3}-\mathrm{k}_{4}}{2} \\
& \mathbf{K} \equiv \mathbf{k}_{1}+\mathbf{k}_{2}=\mathbf{k}_{3}+\mathbf{k}_{\mathbf{4}} \text {. } \\
& \omega_{v} \equiv \omega_{1}+\omega_{2}=\omega_{3}+\omega_{1} . \\
& =\equiv i_{N}-\xi_{K} \equiv i_{i V}-\frac{\hbar^{2} \Lambda^{-2}}{4 m} \text {. } \\
& F_{+}(\mathbf{K} \cdot \mathbf{k}-\mathbf{q}) \equiv 1+f_{B}\left(\epsilon_{\frac{1}{2}} \mathbf{K}+\mathbf{k}-\mathbf{q}\right)+f_{B}\left(\epsilon_{\frac{1}{2}} \mathbf{K}-\mathbf{k}+\mathbf{q}\right) .
\end{aligned}
$$

Here the factor $F_{+}(\mathbf{K} . \mathbf{k}-\mathbf{q})\left(z-2 \epsilon_{\mathbf{k}-\mathbf{q}}+2 \mu\right)^{-1}$ comes from the frequency sum of the product $G_{r}^{(0)} G_{r}^{(0)}$ over $i \omega_{l}$. The function $F_{+}(\mathbf{K} . \mathbf{k}-\mathbf{q})$ incorporates the effect of the Bose statistics obeyed by the atoms involved in the intermediate scattering states.

With this definition of $\Gamma$. the lowest-order diagrams for a dilute Bose-condensed gas in the ladder approximation are shown in Fig.3.-4. which are precisely the diagrams that Popov included in his original paper[32]. The self-energies represented by the diagrams in Fig.3.t can be written explicitly as

$$
\begin{aligned}
& \Sigma_{11}\left(\mathbf{k}, i \omega_{n}\right)=n_{0}\left[\Gamma\left(\frac{\mathbf{k}}{2} \cdot \frac{\mathbf{k}}{2}: \mathbf{k} \cdot i \omega_{n}-\xi_{k}\right)+\Gamma\left(-\frac{\mathbf{k}}{2} \cdot \frac{\mathbf{k}}{2}: \mathbf{k} \cdot i \omega_{n}-\xi_{k}\right)\right] \\
& -\frac{1}{3 V} \sum_{\mathrm{q}, i \omega_{l}} G^{(0)}\left(\mathrm{q}, i \omega_{l}\right)\left[\Gamma\left(\frac{\mathrm{k}-\mathrm{q}}{2} \cdot \frac{\mathrm{k}-\mathrm{q}}{2} ; \mathrm{k}+\mathrm{q} \cdot i \omega_{n}+i \omega_{l}-\xi_{\mathrm{k}+\mathrm{q}}\right)\right. \\
& \left.+\Gamma\left(-\frac{\mathrm{k}-\mathrm{q}}{2} \cdot \frac{\mathbf{k}-\mathbf{q}}{2} ; \mathrm{k}+\mathrm{q} \cdot i \omega_{n}+\omega_{l}-\xi_{\mathrm{k}+\mathrm{q}}\right)\right] \text {. } \\
& \Sigma_{12}\left(\mathbf{k}, i \omega_{n}\right)=n_{0} \Gamma\left(\mathbf{k}, 0 ; 0, i \omega_{n}\right)
\end{aligned}
$$

In comparing this with the self-consistent HFB discussed in Section 3.1. we note there are two differences:

(a) The ladder diagrams are included, replacing the bare interatomic potential v 

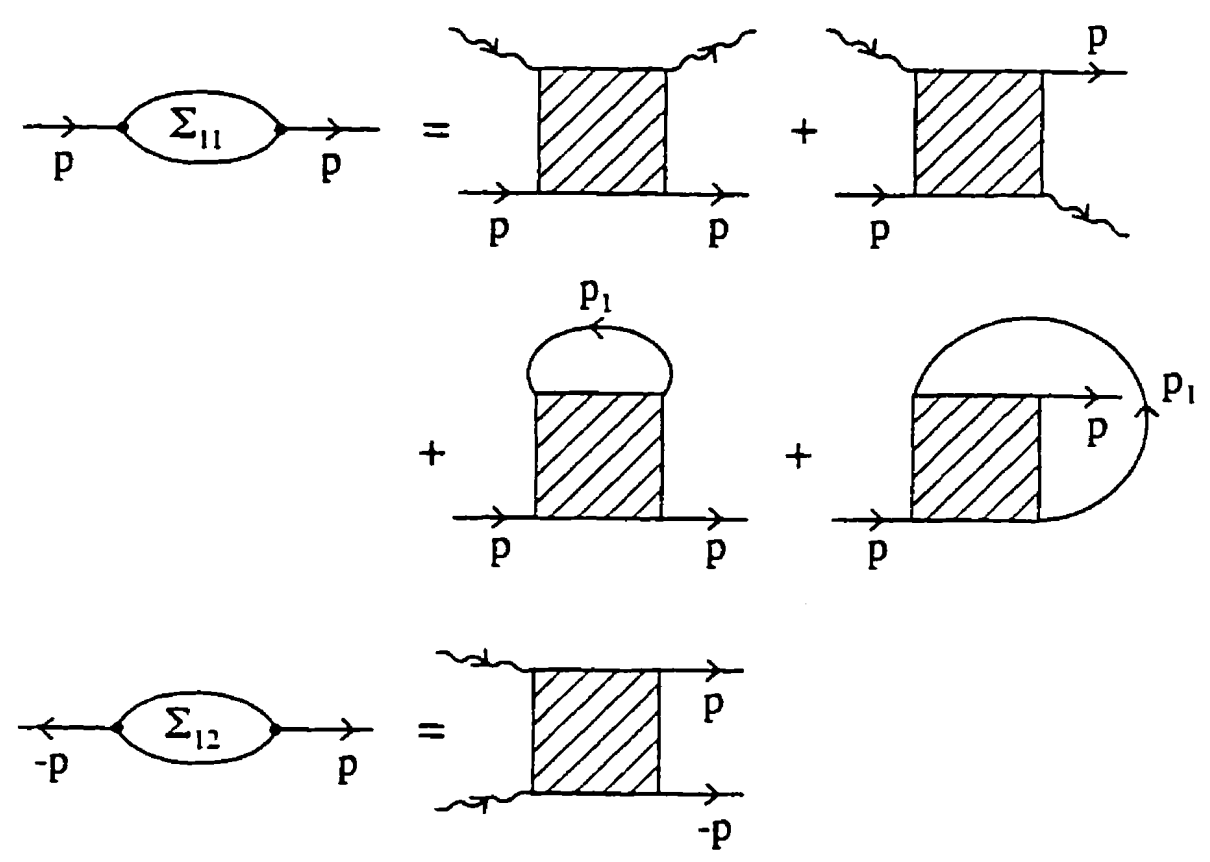

Figure 3.t: Self-energy diagrams in the Popov approximation involving $t$-matrix

by the many-body $t$-matrix $\Gamma$ :

(b) Ideal Bose gas propagators are used in the self-energies. rather than the renormalized ones.

As shown in Appendix A, the many-body $t$-matrix $\Gamma$ can be expressed in terms of the vacuum scattering amplitude $\bar{f}$ defined by

$$
\tilde{f}\left(\mathbf{k}, \mathbf{k}^{\prime}\right)=v\left(\mathbf{k}-\mathbf{k}^{\prime}\right)+\frac{1}{2} \int \frac{d \mathbf{q}}{(2 \pi)^{3}} \frac{v(\mathbf{k}-\mathbf{q})}{\epsilon_{k^{\prime}}-\epsilon_{q}+i \eta} \tilde{f}\left(\mathbf{q} ; \mathbf{k}^{\prime}\right)
$$

We note that our definition of $\tilde{f}$ differs from the ordinary definition of the vacuum scattering amplitude $f$ by a factor of $-4 \pi \hbar^{2} / m$, that is

$$
\tilde{f}\left(\mathbf{k}, \mathbf{k}^{\prime}\right) \equiv-\frac{4 \pi \hbar^{2}}{m} f\left(\mathbf{k}, \mathbf{k}^{\prime}\right)
$$


In the long wavelength limit $\left(|\mathbf{k}|=\left|\mathbf{k}^{\prime}\right| \rightarrow 0\right) . \tilde{f}$ can be shown to reduce to

$$
\dot{f}\left(\mathbf{k} . \mathbf{k}^{\prime}\right)=\frac{t \pi \hbar^{2} a}{m}+O\left(k a^{2}\right)
$$

where $a$ is the s-wave scattering length. The vacuum scattering amplitude $\dot{f}$ describes the effect of the potential on the wavefunction of two atoms in free space. while the many-body $t$-matrix $\Gamma$ describes the similar effect in a medium. that is. in the presence of other atoms in an ideal Bose gas.

It is shown in Appendix $A$ that the momentum dependence of $\Gamma\left(\mathbf{k} \cdot \mathbf{k}^{\prime}: \mathbf{K} . z\right)$ in (3.9) is at least the order of $f^{2}:$ in terms of $a$. this means of order $a^{2}$ [see $\left.(3.19)\right]$. In the first order approximation we are discussing in this chapter. it thus seems reasonable to neglect the momentum dependence of $\Gamma$ in (3.1.5) and (3.16). and keep the self-energies only to first order in $a$. Thus we arrive at

$$
\begin{aligned}
& \Sigma_{11}=2\left(n_{0}+\dot{n}^{(0)}\right) \dot{f}_{0} \\
& \Sigma_{12}=n_{0} \dot{f}_{0} .
\end{aligned}
$$

where $\Gamma$ has been replaced by its value in the limit of $|\mathbf{k}|=\left|\mathbf{k}^{\prime}\right| \rightarrow 0$. that is

$$
\Gamma\left(\mathbf{k} . \mathbf{k}^{\prime}: \mathbf{K} . z\right) \simeq \Gamma(0.0: 0.0)=\dot{f}(0.0)=\frac{4 \pi \hbar^{2} a}{m} \equiv \tilde{f}_{0}
$$

The quantity $\dot{n}^{(0)}$ in (3.20) is the (temperature-dependent) density of excited atoms in a non-interacting Bose gas, given by

$$
\dot{n}^{(0)} \equiv-\frac{1}{3 V} \sum_{\mathbf{k} \neq 0 . i \omega_{n}} e^{i \omega_{n} \eta} C^{(0)}\left(\mathbf{k}, i \omega_{n}\right)=\int \frac{d \mathbf{k}}{(2 \pi)^{3}} \frac{1}{e^{3\left(c_{k}-\mu^{(0)}\right)}-1}
$$

Here. and in the rest of this thesis, we use the superscript " $(0) "$ as a reminder that the quantity is for a non-interacting Bose gas.

In (3.20), we have dropped the $\left(k . i \omega_{n}\right)$ arguments of $\Sigma_{11}$ and $\Sigma_{12}$ because they are now both frequency and momentum-independent. The self-energies in (3.20) will be referred to as the Popov first-order approximation. With it. one can calculate the 
normal and anomalous Green's functions through (2.53) and (2.5t) and use these to evaluate various physical quantities. We now discuss the normal phase $\left(T>T_{\dot{c}}\right)$ and the condensed phase $\left(T<T_{c}\right)$ separately.

\subsection{Normal phase $\left(T>T_{c}\right)$}

In the normal phase. we have $n_{0}=0$ and then $(3.20)$ reduces to

$$
\begin{aligned}
& \Sigma_{11}=2 \dot{n}^{(0)} \dot{f}_{0}, \\
& \Sigma_{12}=0 .
\end{aligned}
$$

Using (3.23) in (2.53) and (2.54), the single-particle Green's functions are

$$
\begin{aligned}
& G_{11}(p)=\frac{1}{i \omega_{n}-\left(\epsilon_{k}-\mu+2 \dot{n}^{(0)} \dot{f}_{0}\right)} . \\
& G_{12}(p)=0
\end{aligned}
$$

With $(3.24)$, the density of the excited atoms as a function of $\mu$ and $T$ is given by

$$
\begin{aligned}
\check{n}(\mu, T) & \equiv-\frac{1}{3 V} \sum_{\mathbf{k} . i \omega_{n}} e^{i \omega_{n} \eta} C_{i_{11}}\left(\mathbf{k} \cdot i \omega_{n}\right) \\
& =\int \frac{d \mathbf{k}}{(2 \pi)^{3}} \frac{1}{e^{3\left(e_{k}-\mu+2 \bar{n}^{(0)}\left(\dot{f}_{0}\right)\right.}-1}
\end{aligned}
$$

If the total density $n$ and the temperature $T$ are given, the chemical potential is determined by the usual condition

$$
\dot{n}(\mu, T)=n \quad(\text { normal system })
$$

Similarly, the chemical potential $\mu^{(0)}$ of a non-interacting Bose gas at $T>T_{\varepsilon}$ is determined by

$$
\tilde{n}^{(0)}\left(\mu^{(0)}, T\right)=n .
$$


Comparing (3.22) and (3.26), one sees that for a given $n$ and $T$. we have

$$
\mu=\mu^{(0)}+2 n \tilde{f}_{0} \quad\left(T>T_{c}\right)
$$

i.e.. the chemical potential is increased by $2 n \tilde{f}_{0}$ due to the effectively repulsive interaction $(a>0)$ between the atoms. Equation (3.29) was derived in Ref.[1.5] in a discussion of BEC in an exciton gas.

The energy spectrum $E_{\mathbf{k}}$ of single-particle excitations (or quasiparticles) in the normal phase is determined by the poles of the Green's function. that is.

$$
E_{\mathbf{k}}=\epsilon_{k}-\mu+2 n \dot{f}_{0}=\epsilon_{k}-\mu^{(0)}
$$

This shows that in the present first-order approximation. the quasiparticles above $T$ : act just like free particles in a non-interacting Bose gas. Because the quasiparticles determine the thermodynamic properties of a system. we conclude that in Popos: approximation. the normal properties of a dilute Bose gas are unchanged compared to that of a non-interacting Bose gas and in particular. the BEC transition temperature $T_{c}$ is the same.

\subsection{Bose-condensed phase $\left(T<T_{c}\right)$}

In the Bose condensed phase. $C_{11}$ and $G_{12}$ are obtained by substituting the self energies (3.20) into the Dyson-Beliaev expressions in (2.53) and (2.5.1):

$$
\begin{aligned}
& G_{11}(p)=\frac{i \omega_{n}+\epsilon_{k}+\Delta}{\left(i \omega_{n}\right)^{2}-\epsilon_{k}^{2}-2 \Delta \epsilon_{k}} \\
& G_{12}(p)=-\frac{\Delta}{\left(i \omega_{n}\right)^{2}-\epsilon_{k}^{2}-2 \Delta \epsilon_{k}}
\end{aligned}
$$

Here the quantity $\Delta$ is defined by

$$
\Delta \equiv \mu-2 \dot{n}^{(0)} \dot{f}_{0},
$$


where the chemical potential $\mu$ has been shown by Popov [31] to satisfy the finitetemperature version of Hugenholtz-Pines theorem given by Hohenberg and Martin $[26]$

$$
\Sigma_{11}(0.0)-\Sigma_{12}(0.0)=\mu
$$

We find using (3.20) and $(3.34)$ that

$$
\mu=2 \dot{n}^{(0)} \dot{f}_{0}+n_{0} \dot{f}_{0} \quad\left(T<T_{\varepsilon}\right)
$$

Combining the last equation with the definition of $\triangle$ in (3.3.3), we arrive at

$$
د(T)=n_{0}(T) \dot{f}_{0}
$$

Here $\lambda$ is written as an explicit function of $T$ to emphasize the dependence of $n_{0}$ on $T$. We note that $د$ is positive (for $a>0$ ) and approaches zero as $n_{0} \rightarrow 0\left(T \rightarrow T_{i}\right.$ ). Both $G_{11}$ and $G_{12}$ in (3.31) and (3.32) have identical poles at $\omega= \pm E_{k}$. where

$$
E_{k}=\sqrt{\epsilon_{k}^{2}+2 \epsilon_{k} \supset(T)}=\sqrt{\epsilon_{k}^{2}+2 \epsilon_{k} n_{0}(T) \dot{f}_{0}}
$$

This gives the energy spectrum of elementary excitations for $T<T_{c}$. This spectrum is formally identical to that of the $T=0$ Bogoliubor approximation in $(2.79)$ if one replaces $r$ by $\tilde{f}_{0}$ (the t-matrix). In contrast to the Bogoliubov model. where $n_{0} \simeq n$, the condensate density $n_{0}(T)$ in the Popov model is strongly dependent on temperature and becomes very small $\left(n_{0}(T) \ll n\right)$ in the vicinity of the BEC phase transition.

On the other hand, one should not over-interpret the formal similarity between the excitation spectrum in the Popov and Bogoliubov models. At the first glance. it seems to imply that one can generalize the Bogoliubov model to finite temperatures simply by taking into account the temperature dependence of $n_{0}$ of an ideal gas. Although this gives the correct excitation spectrum, it gives an incorrect expression for chemical potential, namely $\mu=n_{0}(T) \tilde{f}_{0}$ instead of the correct result in (3.35). This difference was missed in many early attempts to generalize the Bogoliubov theory 
to finite temperatures.

We see that the Popov-Bogoliubov spectra in (3.37) has quite different limits:

$$
\begin{array}{ll}
E_{k} \sim \epsilon_{k}+\Delta(T) & k \gg \hbar^{-1} \sqrt{m \Delta} . \\
E_{k} \sim \hbar c k & k \ll \hbar^{-1} \sqrt{m \Delta} .
\end{array}
$$

$E_{k}$ is free-particle like in the short-wavelength limit. and phonon-like in the longwavelength limit, the phonon velocity $c$ being given by

$$
c \equiv \sqrt{\frac{\Delta(T)}{m}}=\sqrt{\frac{n_{0}(T) \dot{f}_{0}}{m}} .
$$

The single-particle excitation spectrum in (3.39) is proportional to $k$ in the longwavelength limit. As we noted earlier. this linear dependence is expected to be true in any Bose-condensed fluid to all orders in perturbation theory [22. 26]. Although the Popov approximation is only the lowest-order approximation. we see that it gives gapless phonon excitations in the low-energy limit in a Bose gas at finite temperatures. Horeover. $c(T) \rightarrow 0$ as $T \rightarrow T_{c}$. i.e. the phonons in the Popor theory are the "broken-symmetry" soft modes of the Bose-condensed phase. However, unlike at $T=0$. there is no established correspondence between the excitation phonon velocity and the compressional speed of sound at finite temperature. As a side remark. We note that the finite temperature behavior of a Bose liquid like superfluid the is quite different from that of a Bose gas (for a review, see Chapter $T$ of Ref.[8]). In particular. the observed excitation phonon velocity in liquid ${ }^{4} \mathrm{He}$ is found to be essentially temperature-independent right through the superfluid transition.

With the Green's function (3.31). one can now calculate the density of particles in the condensate. For a given total density $n$, we have

$$
n=n_{0}+\tilde{n}
$$


with

$$
\begin{aligned}
\dot{n} & \equiv-\frac{1}{3 V} \sum_{\mathbf{k}, i \omega_{n}} \epsilon^{i \omega_{n} \eta}\left[\frac{i \omega_{n}+\epsilon_{k}+\Delta}{\left(i \omega_{n}-E_{k}\right)\left(i \omega_{n}+E_{k}\right)}\right] \\
& =\int \frac{d \mathbf{k}}{(2 \pi)^{3}}\left[\frac{\epsilon_{k}+\Delta}{2 E_{k}} \operatorname{coth} \frac{3 E_{k}}{2}-\frac{1}{2}\right] \quad\left(T<T_{z}\right)
\end{aligned}
$$

The two equations $(3.41)$ and $(3.42)$ are coupled equations for $n_{0}$. since $\dot{n}$ depends on $E_{k}$ and $\lrcorner$. which are themselves functions of $n_{0}$ [see (3.37) and (3.36)]. In general. such non-linear equations must be solved for numerically. However. in two important special cases, analytical results can be obtained. as we now discuss.

We first consider the low temperature limit $\left(k_{B} T \ll \Delta=n_{0} \dot{f}_{0}\right)$. In this case. the integral in $(3.42)$ can be approximated as

$$
\begin{aligned}
\dot{n} & =\int \frac{d \mathbf{k}}{(2 \pi)^{3}}\left(\frac{\epsilon_{k}+\lambda}{2 E_{k}}-\frac{1}{2}\right)+\int \frac{d \mathbf{k}}{(2 \pi)^{3}} \frac{\epsilon_{k}+\lambda}{E_{k}} f_{B}\left(E_{k}\right) \\
& \simeq \frac{1}{3 \pi^{2}}\left(\frac{m \Delta}{\hbar^{2}}\right)^{3 / 2}+\frac{1}{12}\left(\frac{m}{\hbar^{2}}\right)^{3 / 2}(\Delta)^{-t / 2}\left(k_{B} T\right)^{2} .
\end{aligned}
$$

where in the second step. we have approximated $f_{B}\left(E_{k}\right) \sim \epsilon^{-3 E_{k}}$. With $(3 .+1)$ and $(3.43)$. we obtain

$$
n_{0} \simeq n-\frac{S n_{0}}{3}\left(\frac{n_{0} a^{3}}{\pi}\right)^{1 / 2}-\frac{\mathrm{L}}{\mathrm{l} 2} \frac{m}{\hbar^{3} c}\left(k_{B} T\right)^{2}
$$

where $c$ is the phonon velocity defined in $(3.40)$.

For a low density gas at low temperatures, the second and third terms in $(3.4)$ are small. implying $n_{0} \sim n$. Therefore, when calculating the depletion $\dot{n} \equiv n-n_{0}$ at $T=0$, we can drop the third term and replace $n_{0}$ by $n$ on the right hand side of $(3.44)$ to obtain

$$
\frac{\check{n}}{n} \equiv \frac{n-n_{0}(0)}{n}=\frac{\delta}{3}\left(\frac{n a^{3}}{\pi}\right)^{1 / 2} \ll 1 .
$$

The error introduced by the replacement of $n_{0}$ by $n$ in obtaining (3.4.5) is of higher order in $a$. Eq.(3.45) is the well-known result for the fractional depletion of the condensate in a dilute gas at absolute zero, as first obtained by Bogoliubov [9] in 1947. As a result of interparticle interactions, a small number of particles are exited out of the 
condensate even at $T=0$. For a weakly interacting Bose gas, the fractional depletion is proportional to $\left(n a^{3}\right)^{1 / 2}$. showing that the number of excited atoms increases with density. One expects that a large fraction of the total atoms would occupy the excited states in a strongly interacting Bose system such as liquid ${ }^{4} \mathrm{He}$. In fact. more than $90 \%$ of the atoms in ${ }^{4} \mathrm{He}$ are in excited states at $T=0$. as shown by inelastic neutron scattering experiments and by direct computer simulation studies (for a review. see Chapter 4 of Ref.[s]).

The $T^{2}$ term in (3.44) was first exhibited by Glassgold et al [40] in 1960. Their calculation of finite temperature corrections was based on the use of the $T=0$ Bogoliubov spectrum. and thus is clearly limited to the region $T \sim 0$. Kehr [ [.:3] has also derived this $T^{2}$ correction, with an extra factor $n_{0}(T=0) / n$ :

$$
n_{0}(T)=n_{0}(0)-\frac{1}{12} \frac{n_{0}(0)}{n} \frac{m}{\hbar^{3} c}\left(k_{B} T\right)^{2} .
$$

Kehr's rigorous derivation was based on the structure of the low-lying single-particle excitations of a Bose-condensed system. which are known in all orders of perturbation theory at $T=0$ [2:2]. Therefore, the result (3.46) was not limited to low density or weakly interacting Bose gas. but valid for any Bose fluid at low temperatures. even for liquid ${ }^{4} \mathrm{He}$ where $n_{0}(0) \approx 0.1 n$. In a dilute Bose gas. we have $n_{0}(0) \simeq n$. and then (3.46) reduces to (3.44). We should emphasize that in the limit of ranishing interaction $\left(\tilde{f}_{0} \rightarrow 0\right)$. the results in (3.44) and (3.46) are limited to $T=0$ because these results are derived under the condition $k_{B} T \ll n_{0} \tilde{f}_{0}$.

The $T^{2}$ law for an interacting Bose gas is quite different from the $T^{3 / 2}$ law of an ideal gas as given by [5]

$$
n_{0}(T)=n\left[1-\left(\frac{T}{T_{c}}\right)^{3 / 2}\right]
$$

This reminds us that the introduction of even a small interaction between the particles can drastically change the properties of a Bose gas at low temperatures. where the phonon part of the spectrum [see (3.39)] completely determines the thermodynamics.

As seen from (3.43), the $T$-dependent part of $\tilde{n}$ tends to decrease with increasing value of $\Delta$ given by (3.36), in contrast to the $T=0$ part, which increases with $\Delta$. As 


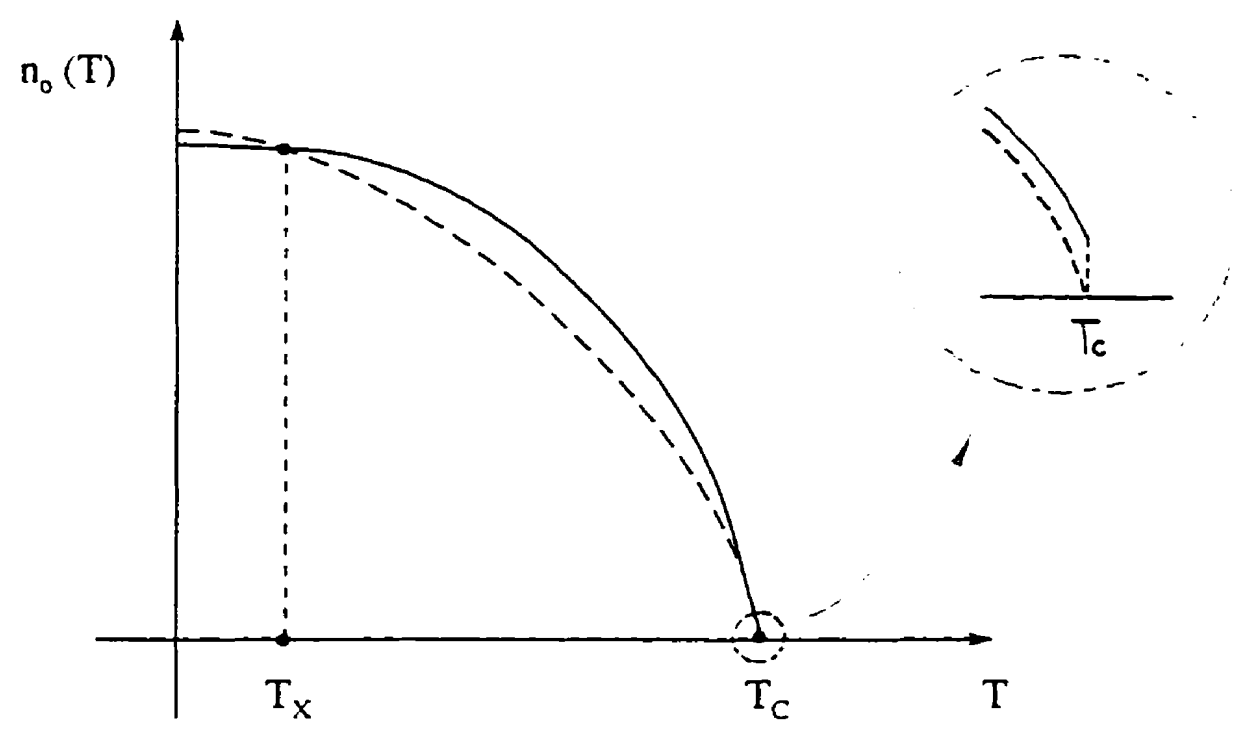

Figure 3.5: Crossover between $n_{0}(T)$ of an interacting gas (solid line) and $n_{0}^{(0)}(T$; of an ideal Bose gas (dashed line). The changes are exaggerated for clarity.

a result, one expects a "crossover" between $n_{0}(T)$ of an interacting gas and $n_{0}^{(0)}(T)$ of a non-interacting gas. as sketched in Fig.3.j. This crossover means that at sufficiently high temperature. the effect of repuisive interactions between the atoms in a dilute weakly-interacting Bose gas is to put more atoms in the condensate. in contrast to the $T=0$ case. It seems to imply that the thermal effect is not as significant in a dilute Bose gas as in an ideal gas. This "crossover" temperature $T_{x}$ can be estimated by comparing $\tilde{n}$ for a weakly-interacting gas in (3.43) with $\tilde{n}^{(0)}$ for an ideal gas the second term at the right hand side of $(3.4 i)]$, yielding

$$
k_{B} T_{x} \sim 0.46 \Delta \sim 0.46 \frac{4 \pi \hbar^{2} a}{m} n
$$

or

$$
T_{\mathbf{x}} / T_{c} \sim 1.74\left(n^{1 / 3} a\right) \ll 1
$$

where the last step follows from (3.i). For example, for $n=2.6 \times 10^{12} \mathrm{~cm}^{-3}$ and $a=53 \AA$ (taken from Ref.[1] for ${ }^{8 \pi} \mathrm{Rb}$ atoms), we estimate that $n^{1 / 3} a=0.0073$ and thus $T_{\mathbf{x}} \sim 0.013 T_{c} \ll T_{c}$. 
We next discuss the region close to the transition. $T \leqslant T_{c}$. It is convenient to calculate the difference $\tilde{n}-n_{c r}$. where $n_{c r}$ is defined by

$$
n_{c r} \equiv \int \frac{d \mathbf{k}}{(2 \pi)^{3}} \frac{1}{\epsilon^{3 \epsilon_{k}}-1}=2.612\left(\frac{m k_{B} T}{2 \pi \hbar^{2}}\right)^{3 / 2}
$$

This is the density of excited atoms (or critical density for the BEC phase transition) of an ideal gas at a given temperature $T[5]$. The dominant contribution to the difference $\bar{n}-n_{c r}$ comes from the energy region where the excitation spectrum $E_{k}$ differs significantly from the free-particle energy $\epsilon_{k}$. which is the region $\epsilon_{k} \sim \Delta$ in our case [see (3.3T)]. We note that $\Delta=n_{0} \tilde{f}_{0}$ is very small near $T_{c}$. namely

$$
\Delta / k_{B} T \sim \Delta / k_{B} T_{c} \ll 1 .
$$

Therefore. we can approximate $\operatorname{coth}\left(3 E_{k} / 2\right)$ by $\left(2 / 3 E_{k}\right)$ in $(3.42)$. and carry out the integral analytically to obtain

$$
\dot{n}-n_{C T} \simeq-\frac{1}{8 \pi}\left(\frac{2 m}{\hbar^{2}}\right)^{3 / 2} k_{B} T(2 \Delta)^{1 / 2}
$$

Combining this result with $(3.41)$. we find

$$
n \simeq n_{0}+n_{c r}-\frac{1}{8 \pi}\left(\frac{2 m}{\hbar^{2}}\right)^{3 / 2} k_{B} T\left(2 n_{0} \tilde{f}_{0}\right)^{1 / 2}
$$

For given $n$ and $T,(3.53)$ is a quadratic equation for $n_{0}$ which has two solutions:

$$
\sqrt{n_{0 . \pm}}=\frac{1}{2}\left[\sqrt{n_{g}(T)} \pm \sqrt{n_{g}(T)+4\left(n-n_{c r}\right)}\right]
$$

Here $\sqrt{n_{g}(T)}$ is the coefficient of $\sqrt{n_{0}}$ in the third term of (3.5.3),

$$
\sqrt{n_{g}(T)} \equiv \frac{1}{8 \pi}\left(\frac{2 m}{\hbar^{2}}\right)^{3 / 2} k_{B} T\left(2 \dot{f}_{0}\right)^{1 / 2}
$$

or

$$
n_{g}(T)=\frac{4 \pi}{2.612^{(4 / 3)}}\left(n^{1 / 3} a\right) n
$$


Since $n>n_{t r}$ below the phase transition temperature. the solution $\sqrt{n_{0 .-}}$ in $(3.54)$ is negative. and thus unphysical. Therefore the condensate density is given by the other solution $n_{0 .+}$. which vields a finite value at $T \rightarrow T_{\varepsilon}^{-}$. namely

$$
n_{0}= \begin{cases}n_{g}\left(T_{c}\right) & T \rightarrow T_{c}^{-} . \\ 0 & T \rightarrow T_{c}^{+} .\end{cases}
$$

This clearly indicates a finite jump in the condensate density $n_{0}$ at the transition point [4t]. This jump is small compared to the total density $n$ in a dilute Bose gas $\left(n^{1 / 3} a \ll 1\right)$, and can be estimated for the ${ }^{87} \mathrm{Rb}$ experiment in Ref. [l] as $6.6 \times 10^{6} \mathrm{~cm}^{-3}$ (compared to $\mathrm{n}=2.6 \times 10^{12} \mathrm{~cm}^{-3}$ ). Nevertheless. the finite jump in the order parameter ( $\sqrt{n_{0}}$. in a Bose fluid with no supercurrent) is the characteristic of a first-order phase transition. Earlier work by Lee, Huang and Yang [24] using a pseudopotential approach led to the same conclusion. This, however, is in contradiction to the fact that $\mathrm{BEC}$ in an interacting Bose system involves a second-order phase transition.

This unphysical behavior near the phase transition point implies that the Popor. approximation breaks down in this region. As emphasized by Popor [31]. the sclfenergies in (3.20) are linear in $\Delta=n_{0} \tilde{f}_{0}$. while the largest diagrams dropped in the Popov approximation are of the order $\left(m / \hbar^{2}\right)^{3 / 2} k_{B} T \tilde{f}_{0} \Delta^{1 / 2}$ (see Chapter 6). As $T$ approaches $T_{c}$ from below, the neglected diagrams become larger than the first-order diagrams. since the former (proportional to $\Delta^{1 / 2}$ ) decrease at a slower rate than $\Delta$. Therefore, the Popov approximation is valid if

$$
\left(\frac{m}{\hbar^{3}}\right)^{3 / 2} k_{B} T \tilde{f}_{0} \Delta^{1 / 2} \ll \Delta
$$

or equivalently.

$$
T_{c}-T \gg\left(n^{1 / 3} a\right) T_{c}
$$

The temperature region defined by $\left(n^{1 / 3} a\right) T_{c}$ is very small in a dilute Bose gas. For example. for the case of ${ }^{87} \mathrm{Rb}$ atoms using densities at the center of the trap given in Ref.[1], the Popov approximation should be applicable when $\left(T_{c}-T\right) \gg 0.0073 T_{c}$.

The properties of a physical system in the neighborhood of the phase transition 
point is always an interesting (and complicated!) problem. However. our major interest in this thesis is not the critical region but the finite-temperature properties of a weakly interacting Bose gas in general. The critical region requires the renomalization group ( $R G$ ) techniques developed to study the behavior of second-order phase transitions. In their recent work. Bijlsma and Stoof [45] have presented a detailed study of a uniform Bose gas using the ingenious application of the RGi approach. I'sing the knowledge of the microscopic details of the interatomic interaction. they were able to calculate the non-universal properties of a dilute Bose gas. In particular. they found that due to interaction effects. the BEC critical temperature could be raised as much as $10 \%$ compared to the ideal gas value for the $\mathrm{Rb}$ and. $\mathrm{Na}$ gases used in recent experimental studies [1, 18]. 


\section{Chapter 4}

\section{The many-body t-matrix}

\section{approximation}

In Chapter 3. we discussed the Popov approximation as the basis of a simple theory which included the thermally-induced depletion of the condensate. The self-energy diagrams which are included in the Popov approximation (see Fig.3.t) were built out of the unperturbed propagator $G^{(0)}$ for a non-interacting Bose gas. As we noted in Chapter 3. the Popov approximation is not valid very close to the phase transition. where it leads to an unphysical discontinuity in the condensate density $n_{0}$. In an attempt to solve this well-known problem. Bijlsma and Stoof [3:3] used a many-body $t$-matrix approximation. based on the use of the self-consistent propagator $C_{r_{11}}$ in the Popov self-energy diagrams. In the present chapter, we examine this many-body t-matrix approximation. In agreement with Bijlsma and Stoof, we show that this $t$ matrix becomes very temperature-dependent and, as one approaches $T_{c}$. it vanishes at the transition point. This leads to a smooth change of $n_{0}$ at $T_{c}$ and thus to the correct order of the phase transition. However, it also shows that $T_{c}$ of an ideal Bose gas is unchanged in the many-body $t$-matrix approximation. The required Green s function formalism for this analysis is the same as we used in Chapter 3. We emphasize only the features that are different from those in the simpler Popov approximation. 


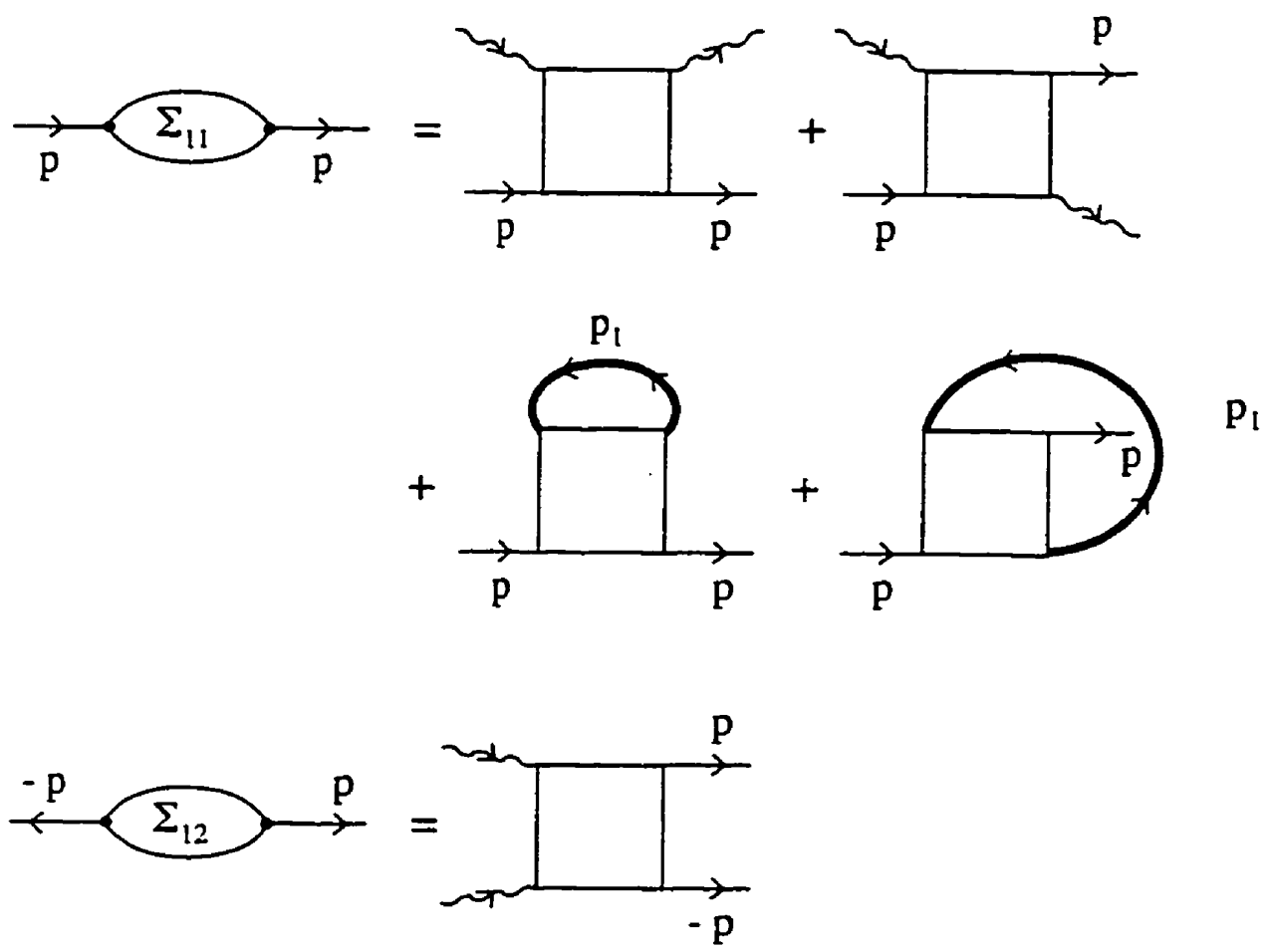

Figure 4.1: Self-energy diagrams in the many-body $t$-matrix approximation.

\subsection{The many-body $t$-matrix}

The self-energy diagrams in the many-body $t$-matrix approximation are shown in Fig.t.1. They look the same as those in Fig.3.t. However. the grey-filled square now represents the multiple scattering of two "dressed" particles, whose propagators $G_{11}$ are to be self-consistently determined. The modified $t$-matrix. denoted as $\tilde{\Gamma}$ to distinguish it from the original $\Gamma$, is defined by the following integral equation [compare with (3.8)]:

$$
\begin{aligned}
\tilde{\Gamma}\left(p_{1}, p_{2} ; p_{3}, p_{4}\right) \equiv v\left(\mathbf{k}_{1}-\mathrm{k}_{3}\right)-\frac{1}{\beta V} \sum_{\mathbf{q}, i \omega_{q}} v(\mathbf{q}) G_{11}\left(p_{1}-q\right) \\
\quad \times G_{11}\left(p_{2}+q\right) \tilde{\Gamma}\left(p_{1}-q, p_{2}+q ; p_{3}, p_{4}\right) .
\end{aligned}
$$

This equation is illustrated by the ladder diagrams in Fig.4.2. In the center-of-mass frame, the many-body $t$-matrix can be written as 


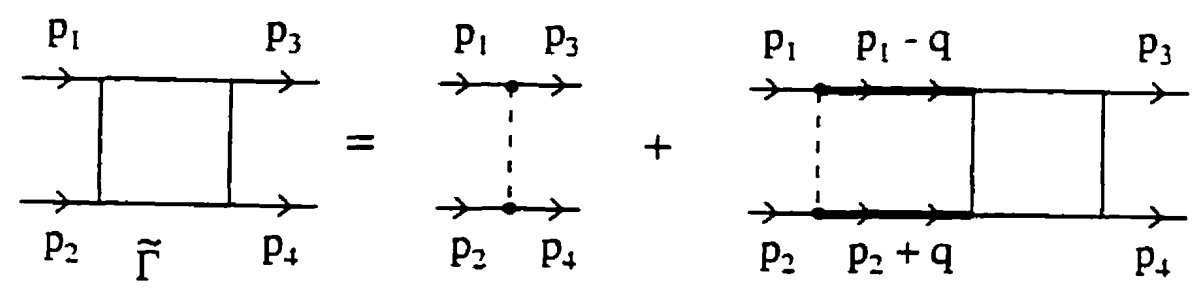

Figure 4.2: Integral equation for the many-body t-matrix $\tilde{\Gamma}$. (A grey-filled square with four "legs" represents $\tilde{\Gamma}$, a solid line with two arrows represents a "dressed" particle or $G_{11}$, and a dashed line represents the interaction potential.)

$$
\begin{aligned}
\tilde{\Gamma}\left(\mathbf{k} \cdot \mathbf{k}^{\prime} \cdot \mathbf{K}: i_{\omega_{N}}-\xi_{K^{-}}\right)= & v\left(\mathbf{k}-\mathbf{k}^{\prime}\right)-\frac{1}{3 V} \sum_{q} v(\mathbf{q}) G_{11}\left(\frac{1}{2} P+p-q\right) \\
& \times G_{11}\left(\frac{1}{2} P-p+q\right) \tilde{\Gamma}\left(\mathbf{k}-\mathbf{q}, \mathbf{k}^{\prime} \cdot \mathbf{K}: i \omega_{\cdot N}-\xi_{K}\right) .
\end{aligned}
$$

Here $\mathbf{k} . \mathbf{k}^{\prime} . \mathbf{K}$. and $\omega_{\mathrm{N}}$ have the same meaning as in the definition of $\Gamma$ in $(3.3)$. We recall that $\xi_{K^{-}}=\hbar^{2} K^{2} / 4 m$ represents the center-of-mass kinetic energy of the scattering pair whose center-of-mass momentum is $\mathbf{K}$.

With the expression of $\tilde{\Gamma}$ in $(4.2)$, the self-energies in the many-body $t$-matrix approximation take the following form [compare with (3.15) and (3.16)]:

$$
\begin{aligned}
\Sigma_{11}\left(\mathbf{k}, i \omega_{n}\right)= & n_{0}\left[\tilde{\Gamma}\left(\frac{\mathbf{k}}{2}, \frac{\mathbf{k}}{2} \cdot \mathbf{k} ; i \omega_{n}-\xi_{k}\right)+\tilde{\Gamma}\left(-\frac{\mathbf{k}}{2}, \frac{\mathbf{k}}{2} \cdot \mathbf{k} ; i \omega_{n}-\xi_{k}\right)\right] \\
& +\sum_{\mathbf{k}_{1}, i \omega_{l}} G_{11}\left(\mathbf{k}_{\mathbf{l}}, i \omega_{l}\right)\left[\tilde{\Gamma}\left(\frac{\mathbf{k}-\mathbf{k}_{\mathbf{l}}}{2}, \frac{\mathbf{k}-\mathbf{k}_{1}}{2}, \mathbf{k}+\mathbf{k}_{1}: i \omega_{n}+i \omega_{l}-\xi_{k+k_{1}}\right)\right. \\
& \left.+\tilde{\Gamma}\left(-\frac{\mathbf{k}-\mathbf{k}_{1}}{2}, \frac{\mathbf{k}-\mathbf{k}_{1}}{2}, \mathbf{k}+\mathbf{k}_{1} ; i \omega_{n}+i \omega_{l}-\xi_{k+k_{1}}\right)\right] \\
\Sigma_{12}\left(\mathbf{k}, i \omega_{n}\right)= & n_{0} \tilde{\Gamma}\left(\mathbf{k}, 0,0 ; i \omega_{n}\right)
\end{aligned}
$$

We are interested in the temperature range where $T$ is not too high compared to $T_{c}$; in other words, we do not consider the case of $T \gg T_{c}$. As is well-known, the energies of important excitations are of the order $k_{B} T$, which is of the order $k_{B} T_{c}$ or smaller 
in the system of interest. We recall that

$$
k_{B} T_{c}=\frac{2 \pi \hbar^{2}}{m}\left(\frac{n}{2.612}\right)^{2 / 3} \sim \frac{\hbar^{2}}{m} n^{2 / 3}
$$

which means that the momenta of important excitations are of the order

$$
k_{i 3} \equiv\left(\frac{2 m k_{B} T_{c}}{\hbar^{2}}\right)^{1 / 2} \sim n^{1 / 3}
$$

One sees that $k_{3}$ is roughly the order of $d^{-1}$. where $d$ is the average distance betwe'n the atoms. For a low density gas such as we are interested in. the average distance between the atoms are much greater than the s-wave scattering length $a$, which implies that $k_{3} \ll a^{-1}$ : in other words. only the low momentum region is important.

Lnder the above condition, we can neglect the energy and momentum dependence of $\tilde{\Gamma}$ in $(4.3)$ and $(4.4)$, arriving at

$$
\begin{aligned}
& \Sigma_{11}=2\left(n_{0}+\tilde{n}^{(1)}\right) \tilde{\Gamma}_{0} . \\
& \Sigma_{12}=n_{0} \tilde{\Gamma}_{0} .
\end{aligned}
$$

Here $\tilde{\Gamma}_{0}$ is defined as

$$
\begin{aligned}
\tilde{\Gamma}_{0} & \equiv \tilde{\Gamma}(0.0 .0: 0) \\
& =v(0)-\frac{1}{3 V} \sum_{\mathbf{q}, i \omega_{l}} v(\mathbf{q}) G_{11}\left(\mathbf{q}, i \omega_{l}\right) G_{11}\left(-\mathbf{q},-i \omega_{l}\right) \tilde{\Gamma}(-\mathbf{q} .0 .0: 0)
\end{aligned}
$$

and $\dot{n}^{(1)}$ is the density of non-condensate atoms.

$$
\tilde{n}^{(1)} \equiv-\frac{1}{\beta V} \sum_{\mathbf{k} \neq 0 . \imath \omega^{\prime} n} e^{i \omega_{n} n} G_{11}\left(\mathbf{k}, i \omega_{n}\right)
$$

Comparing $(4 . \overline{)})$ with $(3.20)$, one sees that the many-body $t$-matrix $\tilde{\Gamma}_{0}$ now replaces the free-space scattering amplitude $\tilde{f}_{0}=4 \pi \hbar^{2} a / m$ in the Popov approximation in Chapter 3. While $\tilde{f}_{0}$ is a constant. $\tilde{\Gamma}_{0}$ depends on the temperature through the many-body propagator $G_{11}$ as shown by (4.8). 
Using $(t . \bar{T})$ in the Dyson's equation (2.53) and (2.5t) for $C_{11}$ and $C_{r_{12}}$. we obtain for $T>T_{c}$.

$$
G_{11}=\frac{1}{i \omega_{n}-\epsilon_{k}+\bar{J}} . \quad C_{12}=0 .
$$

For $T<T_{6}$, we have

$$
\begin{aligned}
& C_{11}=-\frac{i \omega_{n}+\epsilon_{k}+\tilde{J}}{\omega_{n}^{2}+\epsilon_{k}^{2}+2 \tilde{J} \epsilon_{k}} \\
& G_{12}=\frac{\tilde{J}}{\omega_{n}^{2}+\epsilon_{k}^{2}+2 \tilde{J} \epsilon_{k}} .
\end{aligned}
$$

In both cases. the quantity $\tilde{J}$ is defined by

$$
\tilde{J} \equiv \mu-2 \tilde{n}^{(1)} \tilde{\Gamma}_{0}
$$

Using the Hugenholtz-Pines theorem which is valid for $T<T_{c}$. the chemical potential and the quantity $\tilde{\jmath}$ are given by

$$
\left.\begin{array}{l}
\mu=2 \tilde{n}^{(1)} \tilde{\Gamma}_{0}+n_{0} \tilde{\Gamma}_{0} . \\
\bar{J}=n_{0} \tilde{\Gamma}_{0}
\end{array}\right\} \quad\left(T<T_{c}\right)
$$

The quasiparticle excitation energies are determined by the poles of Cireen's functions given by $(4.10)$ and $(4.11)$. We find

$$
E_{k}= \begin{cases}\epsilon_{k}-\tilde{J} & \left(T>T_{c}\right) \\ \sqrt{\epsilon_{k}^{2}+2 \tilde{J} \epsilon_{k}} & \left(T<T_{c}\right)\end{cases}
$$

The density of non-condensate atoms is given by

$$
\begin{aligned}
& \tilde{n}^{(1)}=\int \frac{d \mathbf{k}}{(2 \pi)^{3}} \frac{1}{e^{\beta\left(\epsilon_{k}-\tilde{\Delta}\right)}-1} \quad\left(T>T_{c}\right) \\
& \tilde{n}^{(1)}=\int \frac{d \mathbf{k}}{(2 \pi)^{3}}\left(\frac{\epsilon_{k}+\tilde{\Delta}}{2 E_{k}} \operatorname{coth} \frac{3 E_{k}}{2}-\frac{1}{2}\right) \quad\left(T<T_{c}\right) .
\end{aligned}
$$

With the structure of $G_{11}$ known, we can now examine $\tilde{\Gamma}_{0}$ in more detail. Eq. $(4.8)$ implies that to find $\tilde{\Gamma}_{0}$ requires the knowledge of $\tilde{\Gamma}(-q, 0,0 ; 0)$ for all $q^{2}>0$. not just 
for $q=0$. Since we are interested in a low density Bose gas at temperature $\sim T_{c}$ or lower, the main contribution to the sum over $\mathbf{q}$ in $(4.8)$ comes from the region $q \ll a^{-1}$ [see discussion following (4.4)]. In this case, $v(\mathbf{q})$ and $\tilde{\Gamma}(-\mathbf{q} \cdot 0.0: 0)$ can be approximated by constants $v(0)$ and $\tilde{\Gamma}_{0}$. respectively. We thus arrive at

$$
\tilde{\Gamma}_{0} \simeq v(0)-\frac{1}{3 V} v(0) \tilde{\Gamma}_{0} \sum_{\mathbf{q}, \omega_{n}}^{\prime} C_{11}\left(\mathrm{q}, i \omega_{n}\right) G_{11}\left(-\mathrm{q} .-i \omega_{n}\right)
$$

Here the prime means that the summation is performed only up to a cut-off momentum to avoid the divergence at large $q$.

The ultraviolet divergence in (4.17) is artificially caused by assuming that $v(q)$ and $\widetilde{\Gamma}(-\mathbf{q}, 0,0 ; 0)$ are constant for all $\mathbf{q}$ in $(4.8)$. while in fact these quantities ranish at large $\mathbf{q}$. The uncertainty of the cut-off momentum can be removed by expressing $\tilde{\Gamma}_{0}$ in terms of $\tilde{f}(0.0)$ given by $(3.17)$. A similar procedure leads to

$$
\dot{f}(0.0) \simeq v(0)-\frac{1}{2 V} v(0) \tilde{f}(0.0) \sum_{\mathrm{q}}^{\prime} \frac{1}{\epsilon_{q}}
$$

where the sum over $\mathbf{q}$ is also divergent if performed over the entire $\mathbf{q}$-space.

Fortunately, the underlying source of the divergence at large $\mathbf{q}$ is the same in both $(4.1 T)$ and $(4.18)$. and thus the q-cutoffs are expected to be the same. ( $\operatorname{sing}$ this and $\dot{f}_{0} \equiv \dot{f}(0.0)$, we combine $(4.1 T)$ and $(4.18)$ to find

$$
\tilde{\Gamma}_{0}=\dot{f}_{0}-\alpha(T, \mu) \dot{f}_{0} \tilde{\Gamma}_{0}
$$

or

$$
\tilde{\Gamma}_{0}=\frac{\tilde{f}_{0}}{1+\alpha(T) \tilde{f}_{0}}
$$

where we have defined the function

$$
\alpha(T) \equiv \frac{1}{V} \sum_{\mathrm{q}}^{\prime}\left[\frac{1}{\beta} \sum_{i \omega_{l}} G_{11}\left(\mathrm{q} . i \omega_{l}\right) G_{11}\left(-\mathrm{q} .-i \omega_{l}\right)-\frac{1}{2 \epsilon_{\eta}}\right]
$$

Using (4.10) for $T>T_{c}$ and (4.11) for $T<T_{c}$ in (4.21), we can perform the frequency 
sum to obtain

$$
\alpha(T)= \begin{cases}\int \frac{d \mathbf{k}}{(2 \pi)^{3}}\left(\frac{1}{2 E_{k}} \operatorname{coth} \frac{3 E_{k}}{2}-\frac{1}{2 \epsilon_{k}}\right) & \left(T>T_{c}\right) \\ \int \frac{d \mathbf{k}}{(2 \pi)^{3}}\left[\left(\frac{1}{2 E_{k}}+\frac{\frac{\Delta}{2}^{2}}{\bar{E}_{k}^{3}}\right) \operatorname{coth} \frac{3 E_{k}}{2}-\frac{1}{2 \epsilon_{k}}\right] & \left(T<T_{\varepsilon}\right)\end{cases}
$$

where $E_{k}$ is given by $(4.14)$. The function $\alpha$ is now free of the large-q divergence if the integration is taken over the entire q-space. This justifies our removal of the cutoff in $(4.21)$. The term of order $\tilde{J}^{2}$ in the integrand of $(4.22)$ for the case of $T<T_{i}$ is higher order in $\tilde{\Gamma}_{0}$. This term is omitted (see further remarks at end of Section $t . t$ ).

Eq. $(4.20)$ is still a rather complicated integral equation of $\tilde{\Gamma}_{0}$. since $\alpha$ depends on $E_{k}$ which in turn involves $\tilde{\Gamma}_{0}$. To solve for $\widetilde{\Gamma}_{0}$. we also need to know $\tilde{J}$. which involves the unknown $\mu$ (at $T>T_{c}$ ) or $n_{0}$ (at $T<T_{c}$ ). Therefore $(4.20)$ must be solved in conjunction with

$$
n^{(1)}(\mu . T)+n_{0}=n
$$

Of course. for $T>T_{\llcorner:} n_{0}$ in (4.23) vanishes.

\subsection{Analytical results for the $t$-matrix}

We next consider cases where analytical results for the function $\alpha$ in $(-1.22)$ can be obtained. We first consider the low temperature limit. At $T \rightarrow 0$. the function $\operatorname{coth}\left(3 E_{k} / 2\right) \sim 1+2 \exp \left(-3 E_{k}\right)$. The integral in $(4.22)$ can be carried out to the lowest order in $\tilde{J}\left(=n_{0} \tilde{\Gamma}_{0}\right)$ and $T$ :

$$
\begin{aligned}
a & =\int \frac{d \mathbf{k}}{(2 \pi)^{3}}\left(\frac{1}{E_{k}} \operatorname{coth} \frac{3 E_{k}}{2}-\frac{1}{2 \epsilon_{k}}\right) \\
& \simeq \int \frac{d \mathbf{k}}{(2 \pi)^{3}} \frac{1}{2 E_{k}}\left[\left(1+2 e^{-3 E_{k}}\right)-\frac{1}{\epsilon_{k}}\right] \\
& \simeq-\frac{1}{\pi^{2}}\left(\frac{m}{\hbar^{2}}\right)^{3 / 2}\left(n_{0} \tilde{\Gamma}_{0}\right)^{1 / 2}\left[1-\frac{1}{2}\left(\frac{k_{B} T}{n_{0} \tilde{\Gamma}_{0}}\right)^{2}\right] .
\end{aligned}
$$


Esing this expression for $\alpha$ in (4.19). the low-temperature limit of $\tilde{\Gamma}_{0}$ is given by

$$
\widetilde{\Gamma}_{0}=\tilde{f}_{0}+\frac{1}{\pi^{2}}\left(\frac{m}{\hbar^{2}}\right)^{3 / 2} \tilde{f}_{0}\left(n_{0} \tilde{\Gamma}_{0}^{3}\right)^{1 / 2}\left[1-\frac{1}{2}\left(\frac{k_{B} T}{n_{0} \widetilde{\Gamma}_{0}}\right)^{2}\right]
$$

The solution of $(4.25)$ requires knowledge of $n_{0}(T)$. Using $(4.23)$ and (4.16). we obtain

$$
n \simeq n_{0}+\frac{1}{\pi^{2}}\left(\frac{m}{\hbar^{2}}\right)^{3 / 2} n_{0}\left(n_{0} \tilde{\Gamma}_{0}^{3}\right)^{1 / 2}\left[\frac{1}{3}+\frac{1}{1 \cdot 2}\left(\frac{k_{B} T}{n_{0} \tilde{\Gamma}_{0}}\right)^{2}\right]
$$

which is valid at low temperature $k_{B} T \ll n_{0} \tilde{\Gamma}_{0}$.

For a low density gas at low temperature, the second term in both $(4.25)$ and $(4.26)$ is a small correction to the first term. Therefore. to first order we obtain $\tilde{\Gamma}_{0} \simeq \tilde{f}_{0}$ and $n_{0} \simeq n$. At the next level of approximation. the quantity $n_{0} \tilde{\Gamma}_{0}$ in the higher order terms of $(4.25)$ and $(4.26)$ can be approximated by $n \dot{f}_{0}$. Within this approximation. the depletion given by the second ierm in $(4.26)$ equals that given by $(3.44)$ and $(4.25)$ becomes

$$
\tilde{\Gamma}_{0}=\tilde{f}_{0}\left[1+s\left(\frac{n a^{3}}{\pi}\right)^{1 / 2}-\frac{1}{\pi^{3 / 2}}\left(n a^{3}\right)^{1 / 2}\left(\frac{k_{B} T}{n \dot{f}_{0}}\right)^{2}\right]
$$

Eq. $(-1.27)$ shows that in the low density and low temperature limit $\left[\left(n a^{3}\right)^{1 / 2} \ll 1\right.$ and $\left.k_{B} T \ll n \tilde{f}_{0}\right]$. the $t$-matrix $\tilde{\Gamma}_{0}$ differs very little from $\tilde{f}_{0}$. In this limit the many-body t-matrix approximation reduces to the simpler Popov approximation in Chapter 3.

We next turn to the finite-temperature region just below $T_{c}$. For $T \lesssim T_{c}$, the function $\operatorname{coth}\left(3 E_{k} / 2\right)$ in $(4.22)$ can be approximated as $2 k_{B} T / E_{k}$, yielding

$$
\widetilde{\Gamma}_{0}=\tilde{f}_{0}\left[1-\frac{1}{2 \pi}\left(\frac{m}{\hbar^{2}}\right)^{3 / 2} \frac{\tilde{\Gamma}_{0}}{\left(n_{0} \tilde{\Gamma}_{0}\right)^{1 / 2}} k_{B} T\right]
$$

If we multiply both sides by $n_{0},(4.28)$ becomes an equation of $n_{0} \tilde{\Gamma}_{0}$. which yields the following solution just below $T_{c}$ (where $n_{0}$ is small)

$$
\tilde{\Gamma}_{0}=4 \pi^{2}\left(\frac{\hbar^{2}}{m}\right)^{3} \frac{n_{0}(T)}{\left(k_{B} T_{c}\right)^{2}}
$$




$$
\begin{aligned}
& =\left(\frac{\hbar^{2}}{m}\right)^{3} \frac{4 \pi^{2} n}{\left(k_{B} T_{c}\right)^{2}}\left[1-\left(\frac{T}{T_{c}}\right)^{3 / 2}\right] \\
& =\frac{0.286}{\left(n^{1 / 3} a\right)} \tilde{f}_{0}\left[1-\left(\frac{T}{T_{c}}\right)^{3 / 2}\right] .
\end{aligned}
$$

where the coefficient 0.286 comes from $(2.612)^{4 / 3} / 4 \pi$. In the second line of $(4.29)$. we have made use of the ideal gas condensate density value, $n_{0}(T)=n\left[1-\left(T / T_{\varepsilon}\right)^{3 / 2}\right]$. Eq. $(4.29)$ shows that the $t$-matrix $\tilde{\Gamma}_{0}$ goes to zero in the same way as $n_{0}$ does. This result is confirmed by numerical calculations, in which the self-consistent condensate density $n_{0}(T)$ is used (see Section 4.3 ).

Finally, we consider the region just above $T_{c}$. In this case. we can approximate $(4.15)$ and $(4.22)$ by taking $\operatorname{coth} \beta E_{k} / 2 \sim 2 k_{B} T / E_{k}$. arriving at

$$
\begin{aligned}
n & =n_{c \mathrm{r}}(T)-\frac{1}{4 \pi}\left(\frac{2 m}{\hbar^{2}}\right)^{3 / 2} k_{B} T|\tilde{\Delta}|^{1 / 2} \\
\tilde{\Gamma}_{0} & =\tilde{f}_{0}\left[1-\tilde{\Gamma}_{0}\left(\frac{2 m}{\hbar^{2}}\right)^{3 / 2} \frac{k_{B} T}{8 \pi|\tilde{J}|^{1 / 2}}\right] .
\end{aligned}
$$

Here $n_{\mathrm{cr}} \equiv 2.612\left(m k_{B} T / 2 \pi \hbar^{2}\right)^{3 / 2}$ is the critical density of an ideal Bose gas at a given $T$. I'sing $n=2.612\left(m k_{B} T_{c} / 2 \pi \hbar^{2}\right)^{3 / 2}$. we can rewrite $(4.30)$ in the form

$$
\tilde{J}=-\frac{(2.61 \cdot 2)^{2}}{4 \pi} k_{B} T_{L}\left[\left(\frac{T}{T_{c}}\right)^{3 / 2}-1\right]^{2}
$$

We see that $\tilde{J}$ goes to zero as $T \rightarrow T_{c}$ from above. Using this result for $\tilde{J}$ in $(4.31)$. we obtain

$$
\begin{aligned}
\tilde{\Gamma}_{0} & \simeq\left(\frac{\hbar^{2}}{2 m}\right)^{3 / 2} \frac{8 \pi|\tilde{\Delta}|^{1 / 2}}{k_{B} T_{c}} \\
& =\frac{0.286}{\left(n^{1 / 3} a\right)} \tilde{f}_{0}\left[\left(\frac{T}{T_{c}}\right)^{3 / 2}-1\right]
\end{aligned}
$$

Combining this with (4.29), we see that $\tilde{\Gamma}_{0}$ vanishes as $T$ approaches $T_{c}$ from above or below in a systematic way. How fast $\tilde{\Gamma}_{0}$ approaches zero depends on the value of $\left(n^{1 / 3} a\right)^{-1}$ : the higher the density; the slower it goes to zero. This result is in agreement 


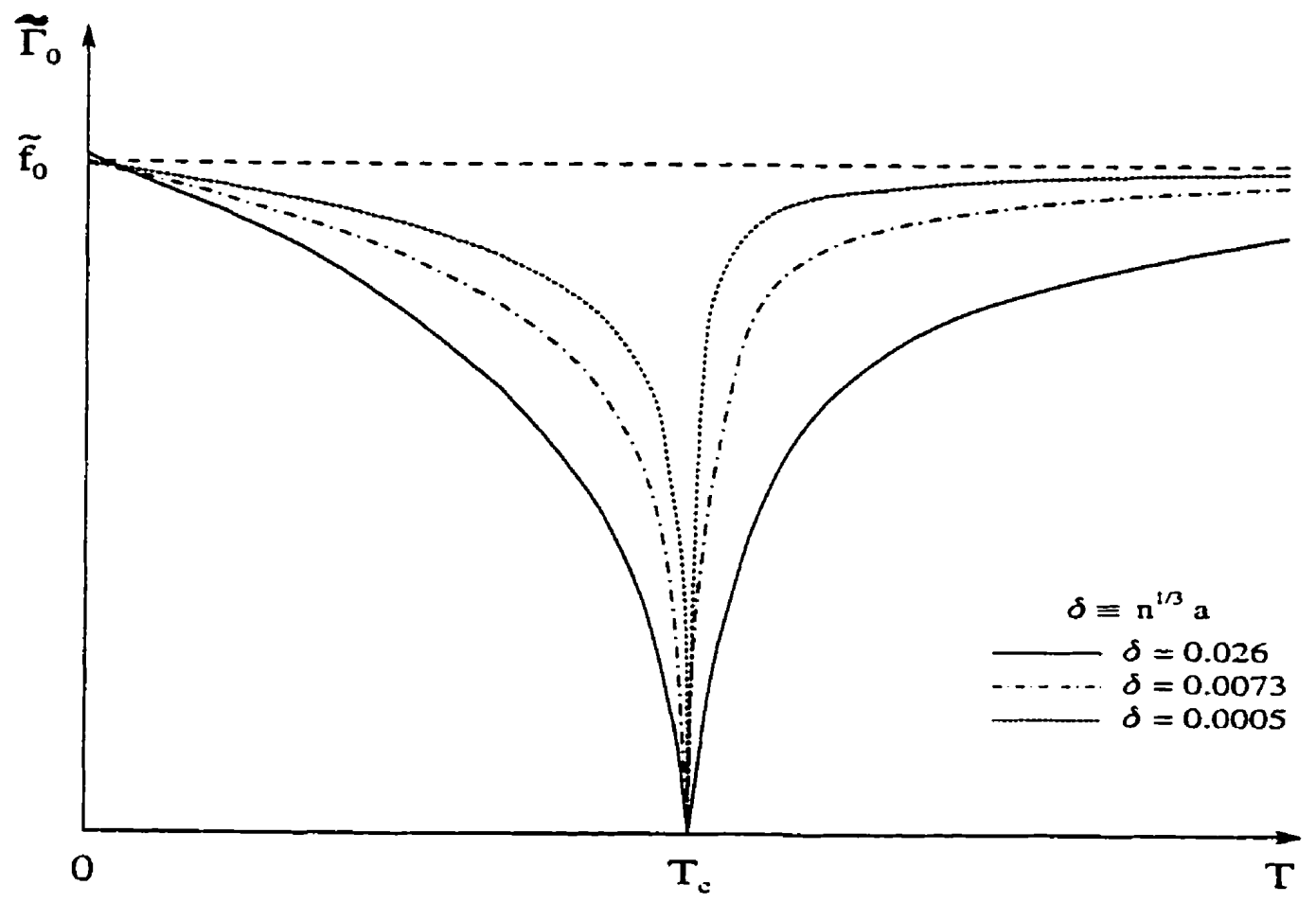

Figure 4.3: Self-consistent $t$-matrix vs. temperature

with that obtained by numerical calculations (see Section 4.3 and Ref. [3:3]). We should note that $(4.29)$ as well as (4.33) are only valid in a small temperature region near $T_{c}$ given by

$$
\left|T-T_{c}\right| \ll\left(n^{1 / 3} a\right) T_{c}
$$

\subsection{Numerical results for the $t$-matrix}

At intermediate temperatures, we must solve the coupled equation for $\tilde{\Gamma}_{0}$ and $E_{k}$ numerically, using (4.13)-(4.16) and (4.19)-(4.21).

In Fig.4.3, we plot $\tilde{\Gamma}_{0}(T)$ as a function of temperature for a given $n$. It goes to zero at the transition temperature and approaches the constant $\tilde{f}_{0}$ at temperatures far away from $T_{c}$. This is in agreement with the analysis given in Section 4.2. $\tilde{\Gamma}_{0} / \tilde{f}_{0}$ also is seen to be slightly greater than unity near $T=0$, due to the correction term $8\left(n a^{3} / \pi\right)^{1 / 2}$ in $(4.29)$. 


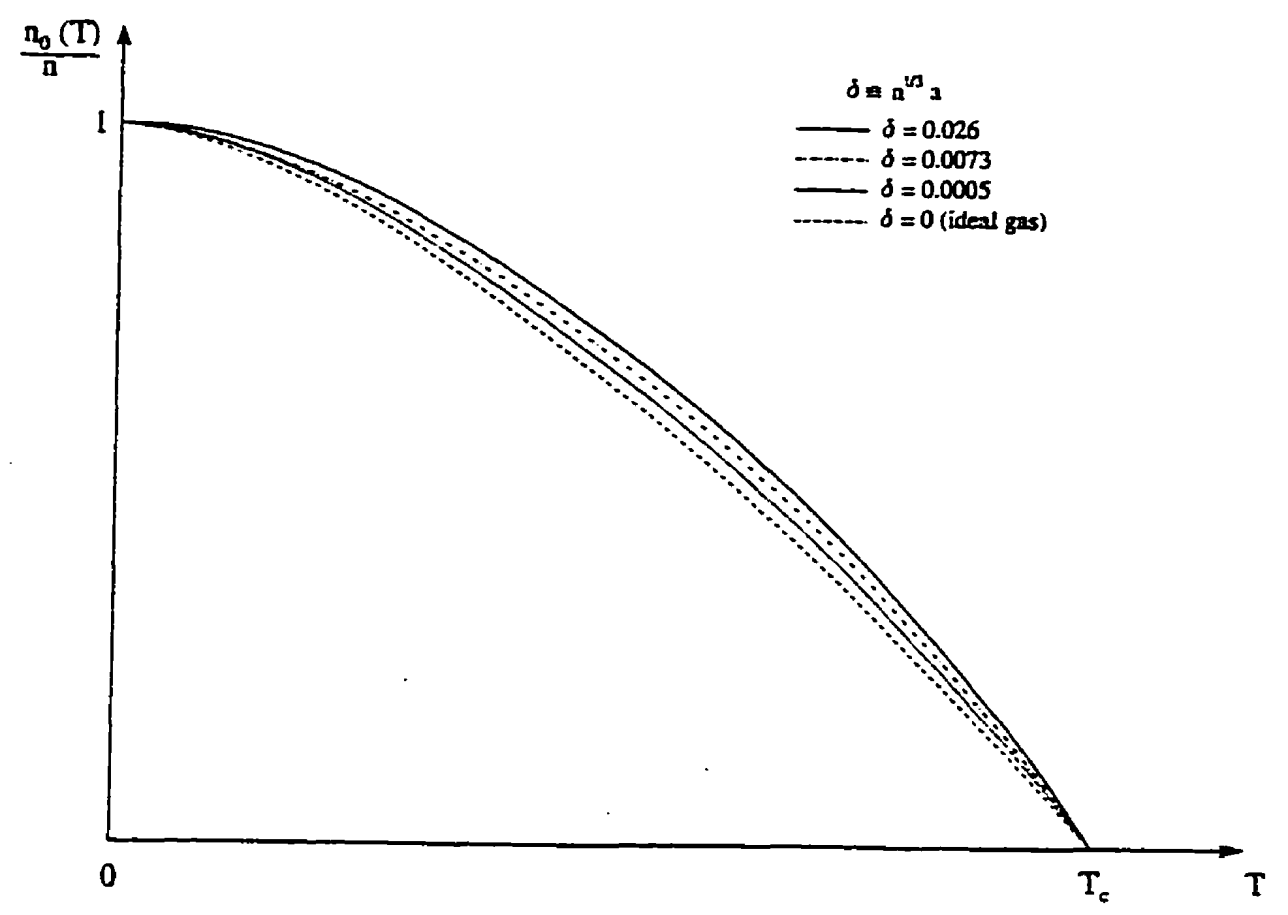

Figure 4.t: Condensate density vs. temperature in the many-body $t$-matrix approximation

We also plot $n_{0}(T)$ in Fig.4.4 for different gases, including a comparison with result for an ideal Bose-condensed gas. We find, at a given temperature. that the condensate fraction is higher in a dilute gas with repulsive interactions than in an ideal Bose gas. However, the situation is opposite near $T=0$. where an ideal Bose gas has a higher condensate fraction. The crossover happens at $T_{\mathrm{x}}$, as discussed at the end of Chapter 3. As expected, the corrections due to interactions are very small. It can be also seen from Fig.4.4 that in the present approximation, $n_{0}(T)$ goes to zero at the BEC transition temperature of an ideal gas. 


\subsection{Assessment of the many-body $t$-matrix ap- proximation}

In this chapter, we have presented a detailed investigation of a dilute Bose gas using the many-body $t$-matrix approximation. We solved the problem analytically at low temperatures as well as near the phase transition point. As far as we know. it is the first time that these analytical results have been obtained. In the limit of $T \rightarrow 0$. the results of the simpler Popov approximation were obtained. In contrast. as $T \rightarrow T_{c}$. the $t$-matrix $\tilde{\Gamma}_{0}$ goes to zero, as explicitly shown by $(4.29)$ and $(4.333)$.

Our numerical results for the many-body $t$-matrix approximation shown in Fig.t.3 and 4.4 are in agreement with those recently obtained by Bijlsma and Stoof [3:3]. Their work was part of a detailed study of thermodynamic properties of a dilute Bose gas. within the framework of a many-body variational approach. Our present calculation expressed in terms of the Popov approximation with a many-body $t$-matrix is less ambitious but perhaps more direct.

In a separate investigation. Bijlsma and Stoof [4.5] have reported a detailed study of how $T_{c}$ of a dilute Bose gas is affected by interactions using a renormalization group $(\mathrm{RG})$ approach. This is based on $\mathrm{RG}$ recursion relations resulting from a systematic integration over the large $\mathbf{k}$ (short distance) correlations, which removes the infrared divergent terms in a systematic way. This more general analysis shows that manybody $t$-matrix approximation is not sufficient near $T_{c}$. This is not unexpected since in using the many-body $t$-matrix in the Popov approximation. one is clearly including a class of higher order self-energy diagrams built out of the normal propagator $G_{11}$. but still omitting any contribution of other higher order diagrams, especially those built out of the anomalous propagators $G_{12}$ and $G_{21}$.

In the succeeding chapters, we shall give a systematic study of the second-order self-energy diagrams first studied by Beliaev [34] at $T=0$ and later by Popov [31] at $T \sim T_{c}$. In particular, we show that these second-order diagrams have contributions which are infrared divergent due to the phonon region of the first-order Popov propagators, as given in Chapter 3. It turns out that the second-order ladder diagram 
is one of these divergent terms and this is associated with $\alpha(T)$ defined in $(4.21)$. It turns out that the term involving $\tilde{J}^{2} / E_{k}^{3}$ in the integrand of $(4.22)$ for $T<\Gamma_{\varepsilon}$. which we have neglected in Section 4.2 and 4.3 . is one such infrared-divergent contribution. Our neglect of this is partially justified by the results of Chapter 6 and $T$. where we show that all such divergent terms cancel out in physical quantities. Still. it is worrisome that the remaining contribution to $\alpha(T)$ is divergent at $T=T_{c}$. One might wonder if this divergent term is also canceled out in a more complete theory. In fact. the RG analysis of Bijlsma and Stoof $[4.5]$ shows that results such as shown in Fig.t.3 based on the many-body $t$-matrix approximation are qualitatively similar to what one obtains in a more complete study. 


\section{Chapter 5}

\section{The second-order Beliaev-Popov (B-P) approximation}

The Beliaev-Popov approximation is the next step beyond the first-order Popor approximation discussed in Chapter 3. Beliaev [34] first studied the higher-order effects of interactions on the properties of a weakly-interacting Bose gas at $T=0$. In his analysis of the same system at finite temperature. Popov [32] argued that Beliaev's second-order self-energy diagrams were still the dominant ones. We call this class of diagrams the Beliaev-Popov (B-P) approximation. This second order approximation treats the coupling between the condensate and non-condensate atoms in an improved fashion and gives rise to a gapless excitation spectrum. It is also the lowest order self-energy approximation which exhibits infrared-divergent contributions.

In this chapter. we give a detailed study of the self-energy diagrams in the B$\mathrm{P}$ approximation, and derive formal temperature-dependent expressions for $\Sigma_{11}$ and $\Sigma_{12}$. In Chapter 6 and 7 , we then use these results to consider various properties in the low frequency, long wavelength limit.

\subsection{Diagrams and their formal expressions}

The diagrams for the diagonal self-energy $\Sigma_{11}$ in the B-P approximation are shown in Fig.5.1. The basic "building block" of these diagrams is the hatched line, representing 

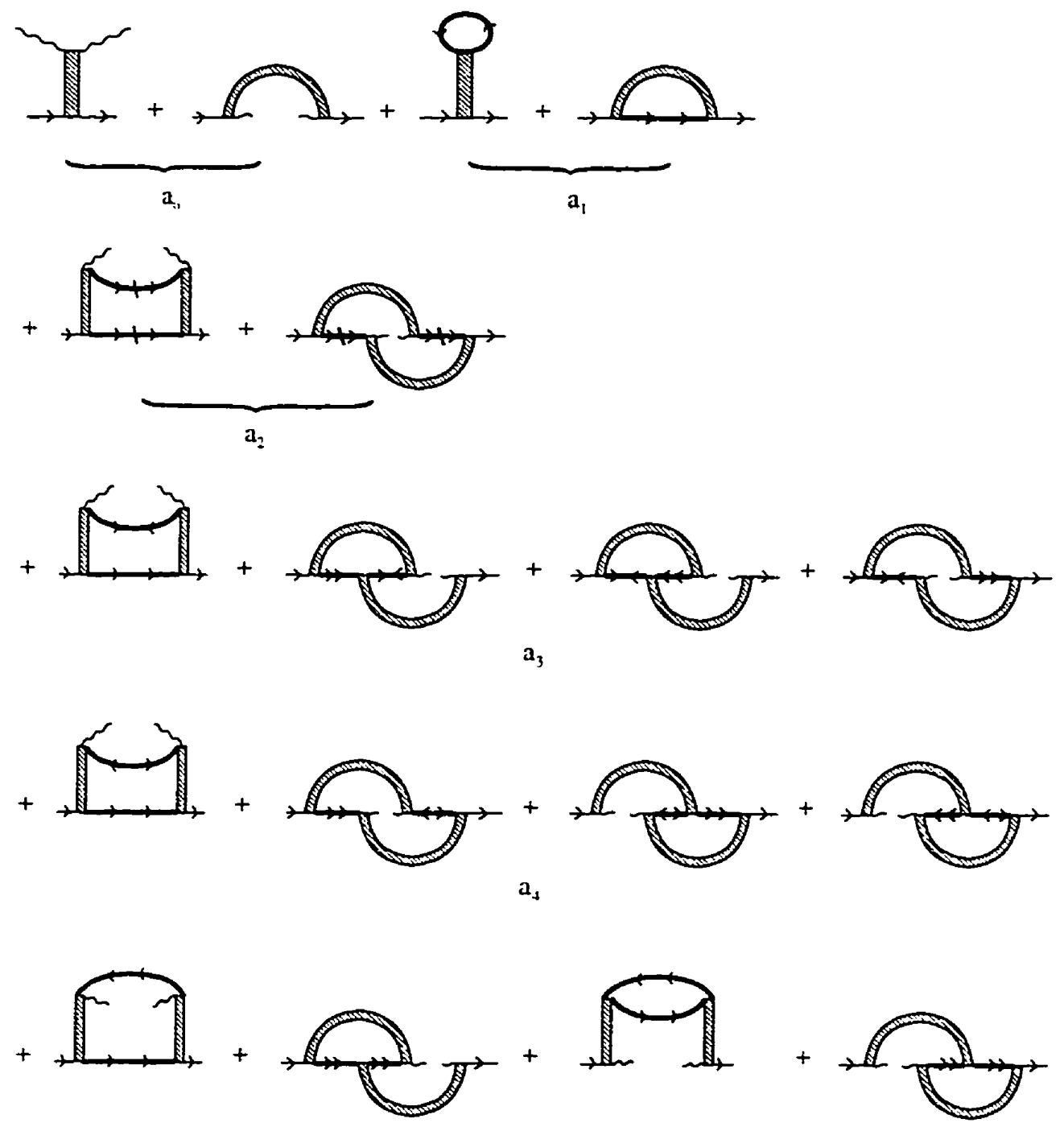

$\mathbf{a}_{5}$

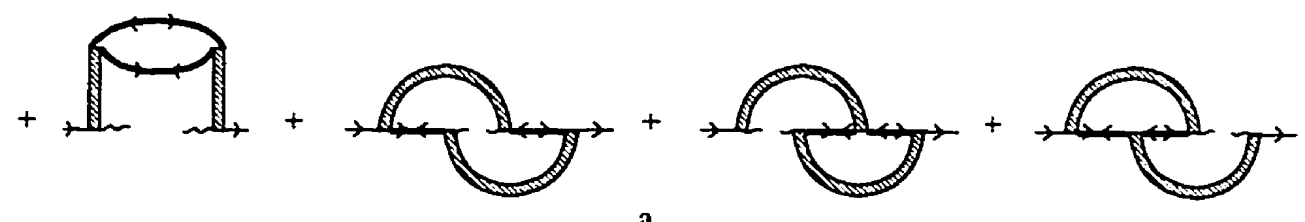

$a_{6}$

Figure 5.1: Diagrams for the diagonal self-energy $\Sigma_{11}$ in the B-P approximation. 


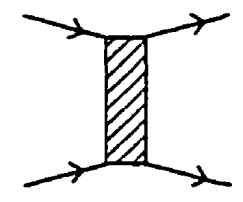

Figure 5.2: An interaction vertex involving a $t$-matrix

the many-body t-matrix $\Gamma$ defined in Eq.(3.8). In order to make it easier to compare with conventional diagrams, we draw $\Gamma$ (represented by a hatched square in Chapter 3) in a way that it resembles an ordinary interaction "line". Each diagram in Fig.j.1 contains at most two $\Gamma$ 's, connected to to each other by elements of the $2 \times 2$ matrix propagator $G$. which includes the normal diagonal propagators $G_{11}$ and $G_{22}$ and the anomalous off-diagonal propagators $G_{12}$ and $G_{21}$. This B-P approximation involves terms explicitly to second-order in $\Gamma$, but it contains the first-order propagators. which are also functions of $\Gamma$, and which can be expanded to arbitrary order in $\Gamma$. In fact, as we shall discuss in Chapter 6, the physical quantities such as the quasiparticle energy in the B-P approximation contain corrections that are the order of $\left(n a^{3}\right)^{1 / 2}$ ( or $\Gamma^{3 / 2}$ ). relative to those in the Popov approximation of Chapter 3.

Following Popov's notation, we divide the diagrams of $\Sigma_{11}$ in Fig.j. 1 into $T$ groups: $a_{0}, a_{1}, a_{2}, \ldots a_{6}$. The first diagram in each group is the "main" diagram: others in the same group are exchange diagrams that can be generated from the main one by exchanging two outgoing arrows or two ingoing arrows (but not both!) associated with interaction vertices. An interaction vertex, shown in Fig.j.2. consists of four "arrows" connecting to an interaction "line" (actually a hatched square representing $\Gamma)$, two of the four "arrows" going in and the other two going out. We now examine each group in $\Sigma_{11}$ in turn:

(1) $a_{0}$ : the diagrams in this group are exactly the Bogoliubov diagrams included in Fig.3.4.

(2) $a_{1}$ : these are the Hartree-Fock diagrams, but now the free propagator $G^{(0)}$ used 
in the first-order Popov approximation has been replaced by the first-order propagator $G_{11}$.

The diagrams in the other groups $\left(a_{2}, \ldots a_{6}\right)$ all involve two $\Gamma^{\prime}$ 's and one pair of condensate lines (represented by a wavy line).

(3) $a_{2}$ : these are built out of two $G_{11}$ 's, both propagating forward. We note that if both of the $G_{11}$ 's are replaced by the lowest-order approximation $C_{-r}{ }^{(0)}$. then $a_{2}$ reduces to $a_{0}$. because by definition, two $G^{(0)}$ 's connecting two $\Gamma^{\circ} \mathrm{s}$ is included in $\Gamma$ itself already. To avoid this double-counting. we must subtract the contribution of $G^{(0)}$ from $a_{2}$. This deduction is represented in Fig.j.l for $a_{2}$ by a stroke across the $C_{11}$ line (following Beliaev's notation [3+]).

(t) $a_{3}$ : these are built out of a $C_{11}$ and a $C_{21}$.

(5) $a_{4}$ : these are built out of a $G_{11}$ and a $G_{12}$.

(6) $a_{5}$ : these are built out of two $G_{11}$ s, one propagating forward. the other propagating backward.

(7) $a_{6}$ : these are built out of a $G_{12}$ and a $G_{21}$.

The diagrams for $\Sigma_{12}$ in the B-P approximation are shown in Fig.5.3. Again following Popov's notation, we have divided these diagrams into seven groups: $b_{0} . b_{1}$. $b_{2}, \ldots b_{6}$. For simplicity, only the main diagram (as defined above) in each group is shown in Fig.j.3. One can easily work out the exchange diagrams in each group. analogous to those shown in Fig.5.1. The total number of diagrams (main one + exchange ones) in each group is denoted by the number in the bracket. Inlike the diagonal self-energy $\Sigma_{11}$, the off-diagonal self-energy diagrams for $\Sigma_{12}$ always contain at least one pair of condensate lines, even though this is implicit in $b_{1}$. As a result, all these diagrams vanish in the normal phase when the condensate density $n_{0}$ vanishes. We list some features of each group in $\Sigma_{12}$ in Fig.5.3:

(1) $b_{0}$ : the diagrams in this group are simply the Bogoliubov diagrams included in the first approximation (see Fig.3.4). 


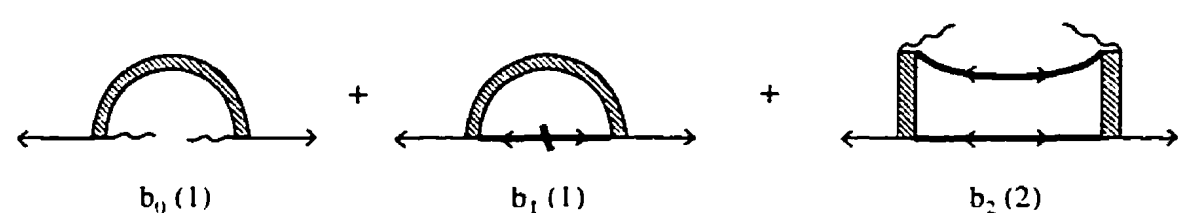

$b_{1}(1)$

$b_{t}(1)$

$b_{2}(2)$

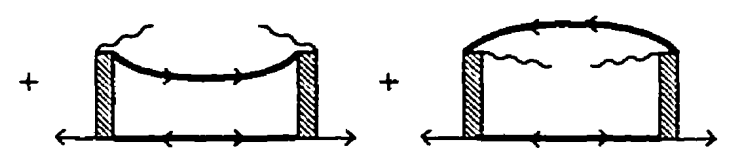

$b_{3}(4) \quad b_{4}(4)$

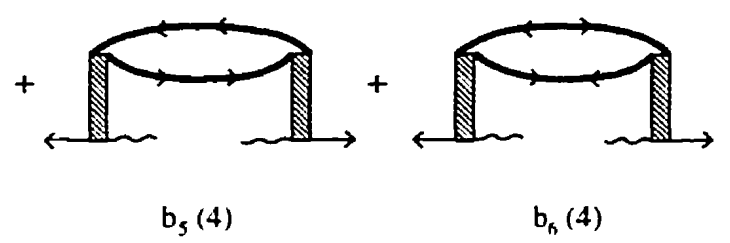

Figure 5.3: Diagrams for the off-diagonal self-energy $\Sigma_{12}$ in the B-P approximation

(2) $b_{1}$ : this involves the anomalous propagator $G_{12}$. As before, the contributions from the lowest-order term of $G_{12}$, given by $n_{0} \tilde{f}_{0} G^{(0)} G^{(0)}$ must be subtracted. for these are already included in $b_{0}$. This deduction is represented by a bold stroke across the $G_{12}$ line.

(3) $b_{2}$ : these are built out of two $G_{12}$ s. and there is one exchange diagram in this group.

(t) $b_{3}$ : these are built out of a $G_{12}$ and a $C_{11}$ which is propagating forward. and there are three exchange diagrams in this group.

(5) $b_{4}$ : these are built out of a $G_{12}$ and a $G_{11}$ which is propagating backward. and there are three exchange diagrams in this group.

(6) $b_{5}$ : these are built out of two $G_{11}$ s, one propagating forward and the other propagating backward, and there are three exchange diagrams in this group.

(i) $b_{6}$ : these are built out of a $G_{12}$ and a $G_{21}$, and there are three exchange diagrams in this group. 
It should be noted that Beliaev [34] and Popov [31] draw their self-energy diagrams using a different definition of vertex functions. Figs.5.1 and 5.3 give the self-energy diagrams in a more conventional, direct manner.

We now work out the explicit mathematical expressions for each of the diagrams in Fig.5.I and 5.3. For convenience, we call the diagrams built with a single $\Gamma$ type-I diagrams $\left(a_{0}, a_{1}, b_{0}, b_{1}\right)$, and denote their contributions to the self-energies as $\Sigma_{11}^{I}$ and $\Sigma_{\mathrm{I} 2}^{\mathrm{I}}$. Similarly, the diagrams built with two $\Gamma$ 's are called type-II diagrams $\left(a_{2} \ldots\right.$ $\left.a_{6}, b_{2} \ldots b_{6}\right)$ and their contributions are denoted as $\Sigma_{11}^{I I}$ and $\Sigma_{12}^{I I}$.

We first examine the type-II diagrams $a_{2} \ldots a_{6}$. and $b_{2}, \ldots b_{6}$. From Appendix $A$. we recall that the many-body $t$-matrix $\Gamma\left(\mathbf{k}, \mathbf{k}^{\prime}, \mathbf{K}: z\right)$ in the limit $|\mathbf{k}|=\left|\mathbf{k}^{\prime}\right| \rightarrow 0$ takes the following value:

$$
\begin{aligned}
\Gamma\left(\mathbf{k}, \mathbf{k}^{\prime} \cdot \mathbf{K}: z\right)= & \tilde{f}_{0}-\frac{i}{4 \pi}\left(\frac{m}{\hbar^{2}}\right) k \tilde{f}_{0}^{2}+\frac{1}{2} \tilde{f}_{0}^{2} \int \frac{d \mathbf{k}_{1}}{(2 \pi)^{3}} \frac{1}{\epsilon_{k_{1}}-\epsilon_{k^{\prime}}-i \eta} \\
& +\tilde{f}_{0}^{2} \int \frac{d \mathbf{k}_{1}}{(2 \pi)^{3}} \frac{1+f_{B}\left(\epsilon_{\mathbf{k}_{1}}\right)+f_{B}\left(\epsilon_{\mathbf{K}-\mathbf{k}_{1}}\right)}{z-2 \epsilon_{\frac{1}{2}} \mathbf{K}-\mathbf{k}_{1}+2 \mu}+O\left(\tilde{f}_{0}^{3}\right) .
\end{aligned}
$$

The integral in the last term in (5.1) comes from the frequency sum over the product of a pair of free propagators, which we define as $J(P)$. namely

$$
\begin{aligned}
J(P) & \equiv \int \frac{d \mathbf{k}_{1}}{(2 \pi)^{3}}\left(-\frac{1}{3}\right) \sum_{i \omega_{1}} G^{(0)}\left(p_{1}\right) G^{(0)}\left(P-p_{1}\right) \\
& =\int \frac{d \mathbf{k}_{1}}{(2 \pi)^{3}} \frac{1+f_{B}\left(\epsilon_{\mathbf{k}_{1}}+f_{B}\left(\epsilon_{\mathbf{K}-\mathbf{k}_{1}}\right)\right.}{z-2 \epsilon_{\frac{1}{2}} \mathbf{K}-\mathbf{k}_{1}+2 \mu}
\end{aligned}
$$

Here $P \equiv\left(\mathbf{K}, i \omega_{N}\right)$ represents the total four-dimensional "momentum" of the two propagators. The terms that are order of $\tilde{f}_{0}^{3}$ or higher are neglected in the B-P self-energies. Therefore, in all type-II diagrams which involve the factor $\Gamma \times \Gamma$. we set $\Gamma=\tilde{f}_{0}$, neglecting the other terms of order $\dot{f}_{0}^{2}$ or higher in (5.1). Within this approximation, the contribution of each exchange diagram equals that of the main diagrams in the same group. Therefore we can simply evaluate the main diagram and multiply the result by the total number of diagrams in each group. The contributions 
of $a_{2} \ldots a_{6}$ in Fig. 5.1 are given by

$$
\begin{aligned}
a_{2}(p)= & 2 n_{0} \tilde{f}_{0}^{2} \int d^{4} p_{1}\left[G_{11}\left(p_{1}\right) G_{11}\left(p-p_{1}\right)\right. \\
& \left.-C_{r}^{(0)}\left(p_{1}\right) G^{(0)}\left(p-p_{1}\right)\right] \\
= & 2 n_{0} \tilde{f}_{0}^{2} \int d^{4} p_{1} G_{11}\left(p_{1}\right) G_{11}\left(p-p_{1}\right)-2 n_{0} \tilde{f}_{0}^{2} J(p) \\
a_{3}(p)= & 4 n_{0} \tilde{f}_{0}^{2} \int d^{4} p_{1} G_{12}\left(p_{1}\right) G_{11}\left(p-p_{1}\right) \\
a_{4}(p)= & 4 n_{0} \tilde{f}_{0}^{2} \int d^{4} p_{1} G_{12}\left(p_{1}\right) G_{11}\left(p-p_{1}\right) \\
a_{5}(p)= & 4 n_{0} \tilde{f}_{0}^{2} \int d^{4} p_{1} G_{11}\left(p_{1}\right) G_{11}\left(p_{1}-p\right) \\
a_{6}(p)= & 4 n_{0} \tilde{f}_{0}^{2} \int d^{4} p_{1} C_{12}\left(p_{1}\right) G_{12}\left(p-p_{1}\right)
\end{aligned}
$$

and those of $b_{2} \ldots b_{6}$ in Fig.5.3 are given by

$$
\begin{aligned}
& b_{2}(p)=2 n_{0} \dot{f}_{0}^{2} \int d^{4} p_{1} C_{12}\left(p_{1}\right) C_{12}\left(p-p_{1}\right): \\
& b_{3}(p)=4 n_{0} \dot{f}_{0}^{2} \int d^{4} p_{1} C_{12}^{\prime}\left(p_{1}\right) C_{11}\left(p-p_{1}\right): \\
& b_{4}(p)=4 n_{0} \dot{f}_{0}^{2} \int d^{4} p_{1} G_{12}\left(p_{1}\right) C_{11}\left(p_{1}-p\right): \\
& b_{5}(p)=4 n_{0} \tilde{f}_{0}^{2} \int d^{4} p_{1} C_{11}\left(p_{1}\right) C_{11}\left(p_{1}-p\right): \\
& b_{6}(p)=4 n_{0} \dot{f}_{0}^{2} \int d^{4} p_{1} C_{12}\left(p_{1}\right) C_{12}\left(p_{1}-p\right) .
\end{aligned}
$$

Here the integral over the four-dimensional momentum $p_{1}$ is defined as usual.

$$
\int d^{4} p_{1} \equiv-\frac{1}{3} \sum_{i \omega_{1}} \int \frac{d^{3} k_{1}}{(2 \pi)^{3}}
$$

We next turn to the type-I diagrams containing a single $\Gamma$. The contribution from the momentum-dependent part of $\Gamma$ is of order $\tilde{f}_{0}^{2}$, as shown in (5.1). Thus we must take into account the momentum-dependence of $\Gamma$ in the type-I diagrams. The contributions of the type-I diagrams are given by

$$
\begin{aligned}
& a_{0}(p)=n_{0}[\Gamma(p, 0 ; p, 0)+\Gamma(p, 0 ; 0, p)] \\
& a_{1}(p)=\int d^{4} p_{1} G_{11}\left(p_{1}\right)\left[\Gamma\left(p, p_{1} ; p, p_{1}\right)+\Gamma\left(p, p_{1} ; p_{1}, p\right)\right]
\end{aligned}
$$




$$
\begin{aligned}
b_{0}(p) & =n_{0} \Gamma(p,-p ; 0.0) . \\
b_{1}(p) & =\int d^{4} p_{1}\left[G_{12}\left(p_{1}\right)-n_{0} \tilde{f}_{0} G^{(0)}\left(p_{1}\right) G^{(0)}\left(-p_{1}\right)\right] \Gamma\left(p .-p: p_{1},-p_{1}\right) \\
& =\int d^{4} p_{1} G_{12} \Gamma\left(p,-p ; p_{1},-p_{1}\right)-n_{0} \tilde{f}_{0}^{2} J(0) .
\end{aligned}
$$

Eqs. (5.3)-(5.12) plus (5.13)-(5.16) include explicit expressions of all the contributions to the self-energies in the B-P approximation. These expressions are slightly different in form from those given by Popov in Chapter 6 of Ref.[31], although the sum of the various diagrams yields the same self-energy in the end. The first-order contributions considered in the Popov approximation [see Eqs.(3.15) and (3.16)] are included in the lowest-order part of $a_{0}, b_{0}$ and $a_{1}$. The other diagrams. $a_{2} \ldots a_{6}$. $b_{1} \ldots b_{6}$ and the higher-order contributions (proportional to $\dot{f}_{0}^{2}$ ) of $a_{0} . b_{0}$ and $a_{1}$. are the new ones. not included in the first-order Popov approximation of Chapter 3. When comparing these higher-order diagrams with those considered by Beliaer [3-4] at $T=0$. we find that they are exactly the same, except for the finite-temperature formalism being used.

We note that at above $T_{c}$. only the Hartree-Fock diagrams $a_{1}$ survive in the B-P approximation. Since the "bubble" in $a_{1}$ involves $G_{1_{1}}$. the non-condensate atoms are actually treated in a "dressed" mean-field manner. Moreover. the condensate atoms are treated in an improved manner compared to the simple Popor approximation. as shown by all the extra terms involving the condensate density $n_{0}$ in Fig.5.1 and 5.3. This leads to a gapless approximation. as proved by the fact that the chemical potential satisfies the Hugenholtz-Pines theorem [34].

It is worthwhile to compare the present B-P approximation with the many-body $t$-matrix approximation discussed in Chapter 4 . The similarities and differences between these two approaches are schematically shown in Fig.5.4. To the first order in $\Gamma$. the self-energy diagrams included in these two approaches are exactly the same. However, the higher order self-energy diagrams are different. For $\Sigma_{11}$, the many-body $t$-matrix approximation includes only $a_{0}, a_{1}$ and $a_{2}$, but not $a_{3}, a_{4}, a_{5}$, and $a_{6}$ : and for $\Sigma_{12}$, the many-body $t$-matrix approximation include only $b_{0}$ and $b_{1}$, but not $b_{2}$, $b_{3}, \ldots, b_{6}$. On the other hand, the many-body $t$-matrix approximation also includes 


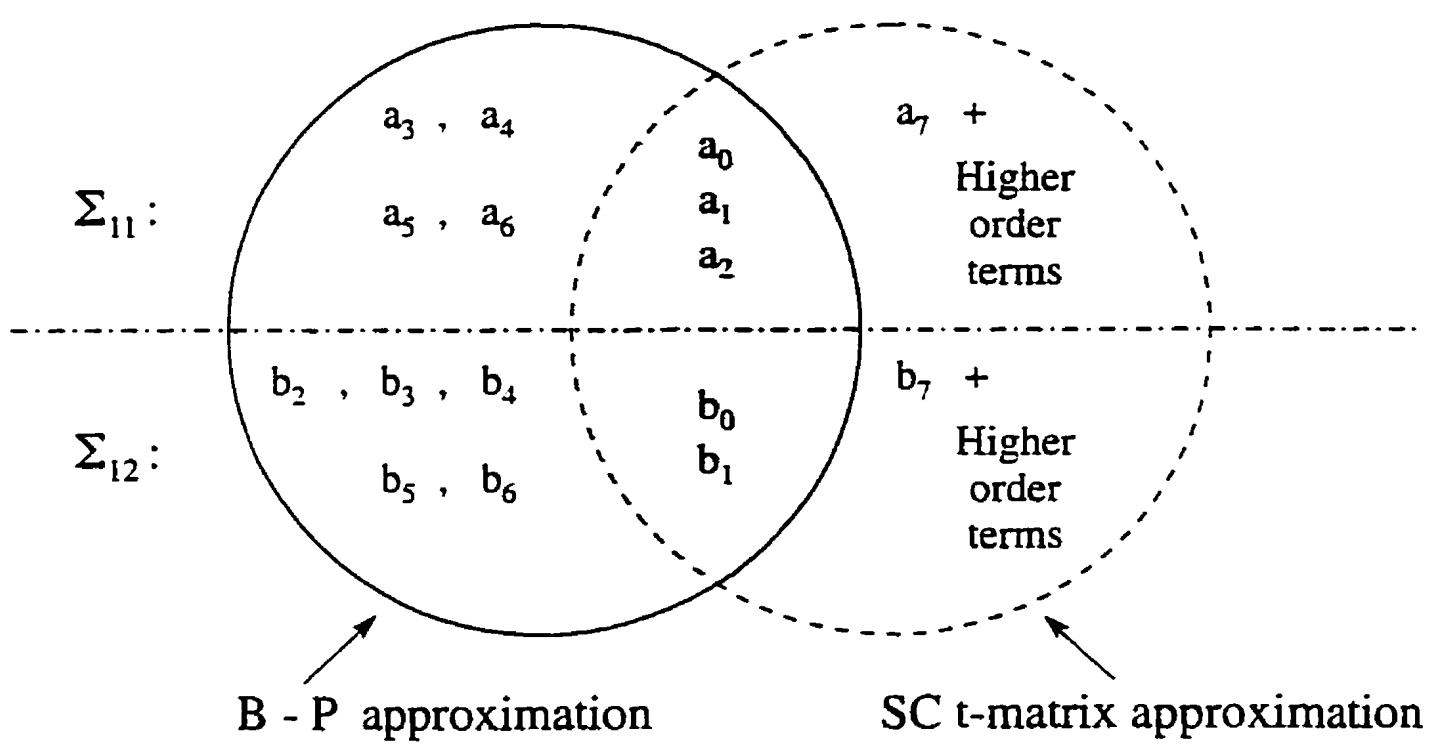

Figure 5.t: Overlap and difference between the many-body $t$-matrix and the B-P approximations.

a set of diagrams, shown in Fig.5.5. that are not included in the B-P approximation. Among these extra diagrams in Fig.j.j. only $a_{7}$ and $b_{7}$ are of order $\Gamma^{2}$.

It is clear that $a_{i}$ is built out of three $G_{11}$ s, two of them propagating forward. the other propagating backward. The explicit expression for $a_{7}$ is

$$
a_{i}(p)=\frac{1}{(\beta V)^{2}} \tilde{f}_{0}^{2} \sum_{q} G_{11}(q) \sum_{p_{1}}\left[G_{11}\left(p_{1}\right) G_{11}\left(p+q-p_{1}\right)-G^{(0)}\left(p_{1}\right) G^{(0)}\left(p+q-p_{1}\right)\right] .
$$

where to avoid double counting, one has to subtract the contribution of $G^{(0)} G^{(0)}$ from $a_{\tau}(p)$. Using the definition

$$
\Gamma^{\mathrm{new}}(p, q ; p, q) \equiv-\frac{1}{\beta V} \tilde{f}_{0}^{2} \sum_{p_{1}} G_{11}\left(p_{1}\right) G_{11}\left(p+q-p_{1}\right)
$$




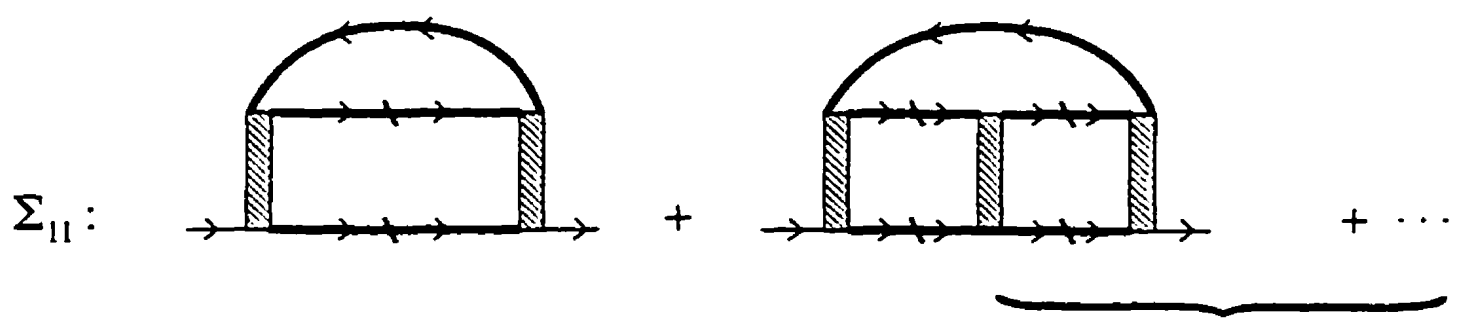

$a_{7}(2)$

Higher order terms

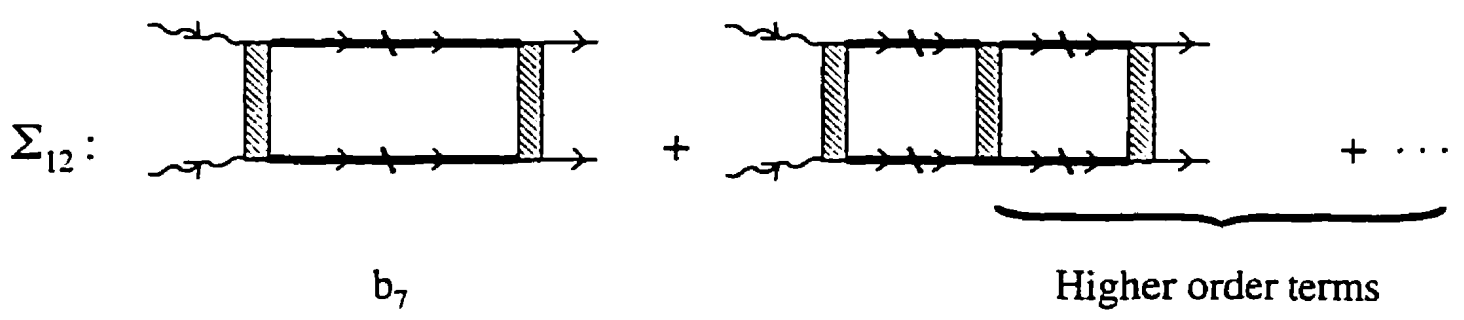

Figure 5.5: Extra diagrams included in the many-body $t$-matrix approximation.

$a_{i}$ is given by

$$
a_{i}(p)=-\frac{1}{\beta V} \sum_{q} G_{11}(q)\left[\Gamma^{\mathrm{new}}(p, q ; p, q)-\Gamma(p, q ; p, q)\right]
$$

Comparing (5.19) with (5.14), we find that the structure of $a_{\tau}$ is similar to that of $a_{1}$. with only $\Gamma^{\text {new }}$ replacing $\Gamma$. The frequency sum in $(5.18)$ cuts off the momentum integral at $k_{\beta} \ll a^{-\mathfrak{l}}$, where the difference between $\Gamma^{\text {new }}$ and $\Gamma$ is expected to be small. This is why $a_{i}$ is not important in the B-P approximation. A similar analysis applies to $b_{7}$ for $\Sigma_{12}$.

It is always difficult to justify why one includes a certain set of higher order diagrams but not the other, and especially so in Bose-condensed systems. We concentrate here on what is done in the B-P approximation, without going into a thorough analysis as whether it is justifiable or not. With a clear understanding of what is included and the consequences, it is then possible to examine such questions. 


\subsection{Evaluation of the B-P self-energies}

In Section 5.1 , we have given formal expressions for all the self-energy diagrams in the $\mathrm{B}-\mathrm{P}$ approximation. We now use these results to obtain more explicit expressions for the self-energies.

It is convenient to write the Green's functions $G_{11}$ and $G_{12}$. given by $(3.31)$ and (3.32). in the following form:

$$
\begin{aligned}
& G_{1 \mathbf{1}}(p)=\frac{A_{\mathbf{k}}}{i \omega_{n}-E_{k}}-\frac{B_{\mathbf{k}}}{i \omega_{n}+E_{k}} . \\
& C_{12}(p)=\frac{C_{\mathbf{k}}}{i \omega_{n}-E_{k}}-\frac{C_{\mathbf{k}}}{i \omega_{n}+E_{k}} .
\end{aligned}
$$

where

$$
\begin{aligned}
A_{\mathbf{k}} \equiv u_{\mathrm{k}}^{2} & =\frac{E_{k}+\epsilon_{k}+\Delta}{2 E_{k}} . \\
B_{\mathbf{k}} \equiv v_{\mathrm{k}}^{2} & =\frac{-E_{k}+\epsilon_{k}+\Delta}{2 E_{k}} . \\
C_{\mathbf{k}} \equiv-\sqrt{A_{\mathrm{k}} B_{\mathrm{k}}} & =-\frac{\Delta}{2 E_{k}} .
\end{aligned}
$$

With these expressions, we perform the Matsubara frequency sums in $(5.3)-(5.7)$ and add $a_{2}$ to $a_{6}$ to obtain

$$
\begin{aligned}
\Sigma_{11}(p)^{\mathrm{II}} \equiv a_{2}(p)+a_{3}(p)+a_{4}(p)+a_{5}(p)+a_{6}(p) \\
=n_{0} \tilde{f}_{0}^{2} \int d^{4} p_{1}\left[2 G_{11}\left(p_{1}\right) G_{1 \mathrm{I}}\left(p-p_{1}\right)+8 G_{12}\left(p_{1}\right) G_{11}\left(p-p_{1}\right)\right. \\
\left.\quad+4 G_{11}\left(p_{1}\right) G_{1 \mathrm{I}}\left(p_{1}-p\right)+4 C_{12}\left(p_{1}\right) G_{12}^{\prime}\left(p-p_{1}\right)\right]-2 n_{0} \tilde{f}_{0}^{2} J(p) \\
=-2 n_{0} \dot{f}_{0}^{2} J(p)+n_{0} \tilde{f}_{0}^{2} \int \frac{d \mathbf{k}_{1}}{(2 \pi)^{2}}\left\{\left[\frac{2 A_{1} A_{2}+8 C_{1} A_{2}+4 A_{1} B_{2}+4 C_{1} C_{2}}{i \omega_{n}-E_{1}-E_{2}}\right] F_{+}\left(E_{1}, E_{2}\right)\right. \\
\left.\left.\quad-\frac{2 B_{1} B_{2}+8 C_{1} B_{2}+4 B_{1} A_{2}+4 C_{1} C_{2}}{i \omega_{n}+E_{1}+E_{2}}\right] F_{-}\left(E_{1}, E_{2}\right)\right\} \\
\quad+\left[\frac{2 B_{1} A_{2}+8 C_{1} A_{2}+4 B_{1} B_{2}+4 C_{1} C_{2}}{i \omega_{n}+E_{1}-E_{2}}\right. \\
\left.\quad-\frac{2 A_{1} B_{2}+8 C_{1} B_{2}+4 A_{1} A_{2}+4 C_{1} C_{2}}{i \omega_{n}-E_{1}+E_{2}}\right]
\end{aligned}
$$


where the two $F_{ \pm}$thermal functions are defined as

$$
\begin{aligned}
& F_{+}\left(E_{1}, E_{2}\right) \equiv f_{B}\left(E_{1}\right)+f_{B}\left(E_{2}\right)+1 \\
& F_{-}\left(E_{1}, E_{2}\right) \equiv f_{B}\left(E_{1}\right)-f_{B}\left(E_{2}\right)
\end{aligned}
$$

$f_{B}(E)$ is the Bose distribution function. Here the subscript " 1 " denotes $\mathrm{k}_{1}$ and $\cdots 2$ " denotes $\mathrm{k}_{2} \equiv \mathrm{k}-\mathrm{k}_{1}$. On the right hand side of (5.26), a variable change of $k_{1}$ to $k_{2}$ interchanges " 1 " and " 2 " only, but has no effect on the integral. Taking advantage of this. we can use $(5.22)-(5.2 t)$ to further simplify $(5.26)$ to

$$
\begin{aligned}
\Sigma_{11}(p)^{\mathrm{II}}= & \frac{1}{2} n_{0} \dot{f}_{0}^{2} \int \frac{d \mathbf{k}_{1}}{(2 \pi)^{3}} \frac{1}{E_{1} E_{2}}\left[\frac{g\left(E_{1}, E_{2}\right)}{i \omega_{n}-E_{1}-E_{2}}-\frac{g\left(-E_{1}-E_{2}\right)}{i \omega_{n}+E_{1}+E_{2}}\right] \\
& +n_{0} \dot{f}_{0}^{2} \int \frac{d \mathbf{k}_{1}}{(2 \pi)^{3}} \frac{1}{E_{1} E_{2}}\left[\frac{g\left(E_{1} \cdot E_{2}\right)}{i \omega_{n}-E_{1}-E_{2}}-\frac{g\left(-E_{1}-E_{2}\right)}{i \omega_{n}+E_{1}+E_{2}}\right. \\
& \left.+\frac{g\left(-E_{1}, E_{2}\right)}{i \omega_{n}+E_{1}-E_{2}}-\frac{g\left(E_{1},-E_{2}\right)}{i \omega_{n}-E_{1}+E_{2}}\right] f_{B}\left(E_{1}\right)-2 n_{0} f_{0}^{2} J(p) .
\end{aligned}
$$

The function $g\left(E_{1}, E_{2}\right)$ is defined as

$$
g\left(E_{1} . E_{2}\right) \equiv 3 \epsilon_{1} \epsilon_{2}-E_{1} E_{2}+\Delta\left(\epsilon_{1}+\epsilon_{2}\right)+د^{2}-\Delta\left(E_{1}+E_{2}\right)+\epsilon_{1} E_{2}+\epsilon_{2} E_{1}
$$

We emphasize that in $(5.26)$. the terms involving $F_{+}$and $F_{-}$contain different physics. The $F_{+}$terms involve creation or destruction of two quasiparticles [poles at

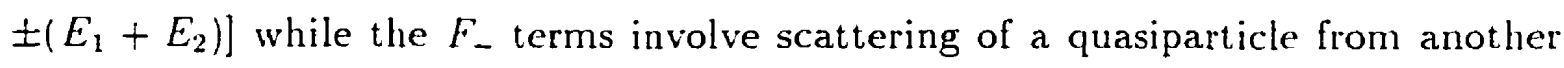
quasiparticle [poles at $\left.\pm\left(E_{1}-E_{2}\right)\right]$. The latter thermal scattering terms vanish at $T=0$. This distinction between these two kinds of terms is less obvious in the expression (5.29).

Similarly, the type-II diagrams of $\Sigma_{12}$ can be added up to give

$$
\begin{aligned}
& \Sigma_{12}(p)^{\mathrm{II}} \equiv b_{2}(p)+b_{3}(p)+b_{4}(p)+b_{5}(p)+b_{6}(p) \\
&=n_{0} \dot{f}_{0}^{2} \int d^{4} p_{1}\left[4 G_{12}\left(p_{1}\right) G_{11}\left(p-p_{1}\right)+4 G_{12}\left(p_{1}\right) G_{11}\left(p_{1}-p\right)\right. \\
&\left.+4 G_{11}\left(p_{1}\right) G_{11}\left(p_{1}-p\right)+6 G_{12}\left(p_{1}\right) G_{12}\left(p-p_{1}\right)\right]
\end{aligned}
$$




$$
\begin{aligned}
= & n_{0} f_{0}^{2} \int \frac{d \mathbf{k}_{1}}{(2 \pi)^{3}}\left\{\left[\frac{4 C_{1} B_{2}+4 C_{1} A_{2}+4 A_{1} B_{2}+6 C_{1} C_{2}}{i \omega_{n}-E_{1}-E_{2}}\right.\right. \\
& \left.-\frac{4 C_{1} B_{2}+4 C_{1} A_{2}+4 B_{1} A_{2}+6 C_{1} C_{2}}{i \omega_{n}+E_{1}+E_{2}}\right] F_{+}\left(E_{1} . E_{2}\right) \\
& +\left[\frac{4 C_{1} B_{2}+4 C_{1} A_{2}+4 B_{1} B_{2}+6 C_{1} C_{2}}{i \omega_{n}+E_{1}-E_{2}}\right. \\
& \left.\left.-\frac{4 C_{1} A_{2}+4 C_{1} B_{2}+4 A_{1} A_{2}+6 C_{1} C_{2}}{i \omega_{n}-E_{1}+E_{2}}\right] F_{-}\left(E_{1}, E_{2}\right)\right\} .
\end{aligned}
$$

A further simplification [compare with $(5.29)]$ leads to

$$
\begin{aligned}
\Sigma_{12}(p)^{I I}= & \frac{1}{2} n_{0} \tilde{f}_{0}^{2} \int \frac{d \mathbf{k}_{1}}{(2 \pi)^{3}} \frac{1}{E_{1} E_{2}}\left[\frac{h\left(E_{1}, E_{2}\right)}{i \omega_{n}-E_{1}-E_{2}}-\frac{h\left(-E_{1},-E_{2}\right)}{i \omega_{n}+E_{1}+E_{2}}\right] \\
& +n_{0} \tilde{f}_{0}^{2} \int \frac{d \mathbf{k}_{1}}{(2 \pi)^{3}} \frac{1}{E_{1} E_{2}}\left[\frac{h\left(E_{1}, E_{2}\right)}{i \omega_{n}-E_{1}-E_{2}}-\frac{h\left(-E_{1},-E_{2}\right)}{i \omega_{n}+E_{1}+E_{2}}\right. \\
& \left.+\frac{h\left(-E_{1}, E_{2}\right)}{i \omega_{n}+E_{1}-E_{2}}-\frac{h\left(E_{1},-E_{2}\right)}{i \omega_{n}-E_{1}+E_{2}}\right] f_{B}\left(E_{1}\right) .
\end{aligned}
$$

where the function $h\left(E_{1}, E_{2}\right)$ is defined as

$$
h\left(E_{\mathrm{i}} \cdot E_{2}\right) \equiv 2 \epsilon_{1} \epsilon_{2}-2 E_{1} E_{2}+\Delta^{2}
$$

The contributions of type-II diagrams are given by $(5.29)-(5.30)$ and $(5.333)-(5.34)$. We next calculate the contribution of the type-I diagrams. C'sing (5.1). $a_{0}(p)$ in (5.1:3) can be expressed in terms of $\tilde{f}_{0}$ as

$$
\begin{aligned}
a_{0}(p) \equiv & a_{0}\left(\mathbf{k}, i \omega_{n}\right)=n_{0}\left[\Gamma\left(\frac{\mathbf{k}}{2} \cdot \frac{\mathbf{k}}{2} \cdot \mathbf{k} ; i \omega_{n}-\xi_{k}\right)+\Gamma\left(\frac{\mathbf{k}}{2} \cdot-\frac{\mathbf{k}}{2} \cdot \mathbf{k} ; i \omega_{n}-\xi_{k}\right)\right] \\
= & 2 n_{0} \tilde{f}_{0}-\frac{i}{4 \pi}\left(\frac{m}{\hbar^{2}}\right) k n_{0} \tilde{f}_{0}^{2}+n_{0} \tilde{f}_{0}^{2} \int \frac{d \mathbf{k}_{1}}{(2 \pi)^{3}} \frac{1}{\epsilon_{k_{1}}-\epsilon_{k / 2}-i \eta} \\
& +2 n_{0} \tilde{f}_{0}^{2} J(p) .
\end{aligned}
$$

It is easy to check that the imaginary part of the integral in $(5.35)$ is given by

$$
n_{0} \tilde{f}_{0}^{2} \int \frac{d \mathbf{k}}{(2 \pi)^{3}} i \pi \delta\left(\epsilon_{q}-\epsilon_{k / 2}\right)=\frac{i}{4 \pi}\left(\frac{m}{\hbar^{2}}\right) k n_{0} \tilde{f}_{0}^{2}
$$


and it exactly cancels the second term of (5.35). We are thus left with

$$
a_{0}(p)=2 n_{0} \tilde{f}_{0}+n_{0} \tilde{f}_{0}^{2} \int \frac{d \mathbf{q}}{(2 \pi)^{3}} \frac{\mathcal{P}}{\epsilon_{q}-\epsilon_{k / 2}}+2 n_{0} \tilde{f}_{0}^{2} J(p)
$$

where $\mathcal{P}$ means the principle value of the integral in (5.37). We note that the term containing $J(p)$ in $(5.37)$ cancels the last term in (5.29). Clearly, the integral in (5.37) is divergent at large q. However. this does not cause any trouble because. as we shall see shortly. this term is canceled by another divergent contribution to $\Sigma_{11}(p)^{\mathrm{II}}$ in $(5.29)$.

For the evaluation of $a_{1}(p)$ in $(5.14)$, we use $(5.1)$ to obtain

$$
\begin{gathered}
a_{1}(p)=\int d^{4} p_{1} G_{11}\left(p_{1}\right)\left[\Gamma\left(\frac{\mathbf{k}-\mathbf{k}_{1}}{2} \cdot \frac{\mathrm{k}-\mathrm{k}_{1}}{2} \cdot \mathbf{k}+\mathrm{k}_{1}: i \omega_{n}+i \omega_{1}-\xi_{\mathbf{k}+\mathrm{k}_{\mathrm{t}}}\right)\right. \\
\left.+\Gamma\left(-\frac{\mathbf{k}-\mathrm{k}_{1}}{2} \cdot \frac{\mathbf{k}-\mathbf{k}_{1}}{2} \cdot \mathrm{k}+\mathrm{k}_{1}: i \omega_{n}+i \omega_{1}-\xi_{\mathbf{k}+\mathrm{k}_{\mathrm{1}}}\right)\right]
\end{gathered}
$$

The main contribution from the four-dimensional momentum integral comes from the region

$$
\left|\mathbf{k}_{1}\right| \sim k_{3} \equiv\left(2 m k_{B} T / h^{2}\right)^{1 / 2}
$$

Since we are only interested in the temperature region that are not too high compared with $T_{c}$, we have $[31]$

$$
\left|\mathbf{k}_{1}\right| \sim\left(\frac{2 m k_{B} T_{c}}{\hbar^{2}}\right)^{1 / 2} \sim n^{1 / 3} \ll a^{-1}
$$

In the small momentum region given by (5.40). the $t$-matrix can be treated as the constant $\tilde{f}_{0}$. The integral of $G_{\mathbf{i}_{1}}\left(p_{1}\right)$ over $p_{1}$ gives the density $\bar{n}^{(1)}$ of excited atoms in the Popov approximation [see (3.42)], namely

$$
\begin{aligned}
\dot{n}^{(1)} & \equiv \int d^{4} p_{1} G_{11}\left(p_{1}\right)=\int \frac{d \mathbf{k}_{\mathbf{l}}}{(2 \pi)^{3}}\left[A_{1} f_{B}\left(E_{1}\right)-B_{1} f_{B}\left(-E_{1}\right)\right] \\
& =\int \frac{d \mathbf{k}_{1}}{(2 \pi)^{3}}\left[\left(A_{1}+B_{1}\right) f_{B}\left(E_{1}\right)+B_{1}\right] \\
& =\int \frac{d \mathbf{k}_{1}}{(2 \pi)^{3}}\left[\frac{\epsilon_{1}+\Delta}{2 E_{1}} \operatorname{coth} \frac{\beta E_{1}}{2}-\frac{1}{2}\right]
\end{aligned}
$$


With $(5.41)$ in $(5.38) . a_{1}$ reduces to

$$
a_{1}(p)=2 \tilde{n}^{(1)} \dot{f}_{0}=2 \dot{f}_{0} \int \frac{d \mathbf{k}_{1}}{(2 \pi)^{3}}\left[\left(A_{1}+B_{1}\right) f_{B}\left(E_{1}\right)+B_{1}\right]
$$

Adding $a_{0}$ and $a_{1}$ to give the contribution from type-I diagrams of $\Sigma_{11} \cdot(5.37)$ and $(5 .+2)$ give

$$
\begin{aligned}
\Sigma_{11}^{I} & \equiv a_{0}(p)+a_{1}(p) \\
& =2\left(n_{0}+\dot{n}^{(1)}\right) \tilde{f}_{0}+n_{0} \tilde{f}_{0}^{2} \int \frac{d \mathbf{k}_{\mathbf{i}}}{(2 \pi)^{3}} \frac{\mathcal{P}}{\epsilon_{k_{1}}-\epsilon_{k / 2}}+2 n_{0} \tilde{f}_{0}^{2} J(p)
\end{aligned}
$$

The two anomalous type-I diagrams are $b_{0}$ and $b_{1}$. With (5.1) and (5.1.5). $b_{0}$ is given by

$$
\begin{aligned}
b_{0}(p) & =n_{0} \Gamma(\mathbf{k} \cdot 0.0 ; 0) \\
& =n_{0} \tilde{f}_{0}+\frac{1}{2} n_{0} \tilde{f}_{0}^{2} \int \frac{d \mathbf{k}_{1}}{(2 \pi)^{3}} \frac{\mathcal{P}}{\epsilon_{k_{1}}-\epsilon_{k / 2}}+n_{0} \tilde{f}_{0}^{2} J(0)
\end{aligned}
$$

In evaluating $b_{1}$. we recall the fact that the lowest-order of $C_{12}$ is proportional to $n_{0} \dot{f}_{0} C_{r}^{(0)} G_{r}^{(0)}$. From (j.16). it is clear that $b_{1}$ is at least the order of $\dot{f}_{0}^{2}$. therefore we can neglect the momentum dependence of $\Gamma$ in $(5.1)$ and set it equal to $\dot{f}_{0}$. Within this approximation. $b_{1}$ is given by

$$
\begin{aligned}
b_{1}(p) & =\tilde{f}_{0} \int d^{4} p_{1}\left[G_{12}\left(p_{1}\right)-n_{0} \dot{f}_{0} C_{r}^{(0)}\left(p_{1}\right) G_{r}^{(0)}\left(-p_{1}\right)\right] \\
& =\dot{f}_{0} \int \frac{d \mathbf{k}_{1}}{(2 \pi)^{3}}\left(-\frac{1}{3}\right) \sum_{i \omega_{1}} \frac{n_{0} \dot{f}_{0}}{\left(i \omega_{i}-E_{1}\right)\left(i \omega_{1}+E_{1}\right)}-n_{0} \dot{f}_{0}^{2} J(0) \\
& =-\frac{1}{2} n_{0} \dot{f}_{0}^{2} \int \frac{d \mathbf{k}_{1}}{(2 \pi)^{3}} \frac{1}{E_{1}} \operatorname{coth} \frac{3 E_{1}}{2}-n_{0} \tilde{f}_{0}^{2} J(0) .
\end{aligned}
$$

Using (5.44) and (5.45), the sum of $b_{0}$ and $b_{1}$ gives the type-I diagram contribution to $\Sigma_{12}$ :

$$
\begin{aligned}
\Sigma_{12}^{\mathrm{I}} & \equiv b_{0}(p)+b_{1}(p) \\
& =n_{0} \dot{f}_{0}+\frac{1}{2} n_{0} \tilde{f}_{0}^{2} \int \frac{d \mathbf{k}_{1}}{(2 \pi)^{3}}\left[\frac{\mathcal{P}}{\epsilon_{k_{1}}-\epsilon_{k / 2}}-\frac{1}{E_{1}} \operatorname{coth} \frac{\beta E_{1}}{2}\right]
\end{aligned}
$$


We note that the terms involving $J(0)$ in $(5.44)$ and $(5.45)$ do not show up in $b_{0}+b_{1}$ because they cancel each other exactly.

This completes our evaluation of type-I and type-II diagrams. Now we combine the contributions of the two types to find $\Sigma_{11}$ and $\Sigma_{12}$. By combining (5.29) and (5.43), we obtain the following expression for $\Sigma_{11}$,

$$
\begin{aligned}
\Sigma_{1 \mathrm{l}}(p)= & \Sigma_{1 \mathrm{l}}^{\mathrm{I}}(p)+\Sigma_{1 \mathrm{l}}^{\mathrm{II}}(p) \\
= & 2 n_{0} \dot{f}_{0}+2 \dot{f}_{0} \int \frac{d \mathbf{k}_{\mathbf{l}}}{(2 \pi)^{3}} B_{1}+n_{0} \dot{f}_{0}^{2} \int \frac{d \mathbf{k}_{1}}{(2 \pi)^{3}} \frac{\mathcal{P}}{\epsilon_{1}-\epsilon_{k / 2}} \\
& +\frac{1}{2} n_{0} \dot{f}_{0}^{2} \int \frac{d \mathbf{k}_{1}}{(2 \pi)^{3}} \frac{1}{E_{1} E_{2}}\left[\frac{g\left(E_{1}, E_{2}\right)}{i \omega_{n}-E_{1}-E_{2}}-\frac{g\left(-E_{1}-E_{2}\right)}{i \omega_{n}+E_{1}+E_{2}}\right] \\
& +n_{0} \dot{f}_{0}^{2} \int \frac{d \mathbf{k}_{1}}{(2 \pi)^{3}} \frac{1}{E_{1} E_{2}}\left[\frac{g\left(E_{1}, E_{2}\right)}{i \omega_{n}-E_{1}-E_{2}}-\frac{g\left(-E_{1} \cdot-E_{2}\right)}{i \omega_{n}+E_{1}+E_{2}}+\frac{g\left(-E_{1}, E_{2}\right)}{i \omega_{n}+E_{1}-E_{2}}\right. \\
& \left.-\frac{g\left(E_{1} \cdot-E_{2}\right)}{i \omega_{n}-E_{1}+E_{2}}\right] f_{B}\left(E_{1}\right)++2 \tilde{f}_{0} \int \frac{d \mathbf{k}_{1}}{(2 \pi)^{3}}\left(A_{1}+B_{1}\right) f_{B}\left(E_{1}\right) .
\end{aligned}
$$

where the function $g$ is defined in (5.30). Similarly, combining (5.33) and (5.46). we obtain

$$
\begin{aligned}
\Sigma_{\mathrm{i} 2}(p)= & \Sigma_{11}^{\mathrm{I}}(p)+\Sigma_{12}^{\mathrm{II}}(p) \\
= & n_{0} \dot{f}_{0}+\frac{1}{2} n_{0} \tilde{f}_{0}^{2} \int \frac{d \mathbf{q}}{(2 \pi)^{3}}\left[\frac{\mathcal{F}}{\epsilon_{q}-\epsilon_{k / 2}}-\frac{1}{E_{q}}\right] \\
& +\frac{1}{2} \int \frac{d \mathbf{k}_{1}}{(2 \pi)^{3}} \frac{1}{E_{1} E_{2}}\left[\frac{h\left(E_{1}, E_{2}\right)}{i \omega_{n}-E_{1}-E_{2}}-\frac{h\left(-E_{1},-E_{2}\right)}{i \omega_{n}+E_{1}+E_{2}}\right] \\
& +n_{0} \tilde{f}_{0}^{2} \int \frac{d \mathbf{k}_{1}}{(2 \pi)^{3}} \frac{1}{E_{1} E_{2}}\left[\frac{h\left(E_{1}, E_{2}\right)}{i \omega_{n}-E_{1}-E_{2}}-\frac{h\left(-E_{1},-E_{2}\right)}{i \omega_{n}+E_{1}+E_{2}}+\frac{h\left(-E_{1}, E_{2}\right)}{i \omega_{n}+E_{1}-E_{2}}\right. \\
& \left.-\frac{h\left(E_{1},-E_{2}\right)}{i \omega_{n}-E_{1}+E_{2}}\right] f_{B}\left(E_{1}\right)-n_{0} \tilde{f}_{0}^{2} \int \frac{d \mathbf{q}}{(2 \pi)^{3}} \frac{f_{B}\left(E_{q}\right)}{E_{q}} .
\end{aligned}
$$

where the function $h$ is defined in (5.34).

In (5.47) and (5.48), we have deliberately separated out the part that does not involve the Bose factor and the part that is explicitly temperature-dependent. The first two lines in each expression gives the former contribution, which we define as 
$\Sigma^{0}$

$$
\begin{aligned}
\Sigma_{11}^{0}(p) & \equiv 2 n_{0} \tilde{f}_{0}+2 \tilde{f}_{0} \int \frac{d \mathbf{k}_{1}}{(2 \pi)^{3}} B_{1}+n_{0} \tilde{f}_{0}^{2} \int \frac{d \mathbf{k}_{1}}{(2 \pi)^{3}} \frac{\mathcal{P}}{\epsilon_{1}-\epsilon_{k / 2}} \\
& +\frac{1}{2} n_{0} \tilde{f}_{0}^{2} \int \frac{d \mathbf{k}_{1}}{(2 \pi)^{3}} \frac{1}{E_{1} E_{2}}\left[\frac{g\left(E_{1}, E_{2}\right)}{i \omega_{n}-E_{1}-E_{2}}-\frac{g\left(-E_{1}-E_{2}\right)}{i \omega_{n}+E_{1}+E_{2}}\right] \\
\Sigma_{1 \cdot 2}^{0}(p) & \equiv n_{0} \dot{f}_{0}+\frac{1}{2} n_{0} \tilde{f}_{0}^{2} \int \frac{d \mathbf{q}}{(2 \pi)^{3}}\left[\frac{\mathcal{P}}{\epsilon_{q}-\epsilon_{k / 2}}-\frac{1}{E_{q}}\right] \\
& +\frac{1}{2} \int \frac{d \mathbf{k}_{1}}{(2 \pi)^{3}} \frac{1}{E_{1} E_{2}}\left[\frac{h\left(E_{1}, E_{2}\right)}{i \omega_{n}-E_{1}-E_{2}}-\frac{h\left(-E_{1}-E_{2}\right)}{i \omega_{n}+E_{1}+E_{2}}\right]
\end{aligned}
$$

These expressions correspond to Beliaev's results at $T=0$. as given in Eq. $(5.9)$ and (5.10) of Ref.[34]. However. when used at finite temperatures. $\Sigma^{0}$ is implicitly temperature-dependent through its dependence on the condensate density $n_{0}(T)$.

\subsection{Energy of excitations in the B-P approxima- tion}

Once we have obtained a specific approximation for $\Sigma_{11}$ and $\Sigma_{12}$. we can substitute these into (2.53) and (2.54) to calculate the Green's functions at finite temperatures. Due to the lengthy expressions in $(5.4 \pi)$ and (5.45). the results for $C_{11}$ and $C_{12}$ are very complicated. However, there is a way [34] to simplify such results so that the poles (and their residues) of $G_{11}$ and $G_{12}$ are more clearly exhibited. and the secondorder corrections to the energy of excitations are shown in an explicit manner. To do so. it is convenient to separate out contributions which are explicitly linear and quadratic in $\dot{f}_{0}$,

$$
\begin{aligned}
\Sigma_{11}(p) & =\Sigma_{11}^{(1)}(p)+\Sigma_{11}^{(2)}(p) . \\
\Sigma_{12}(p) & =\Sigma_{12}^{(1)}(p)+\Sigma_{12}^{(2)}(p) . \\
\mu & =\mu^{(1)}+\mu^{(2)} .
\end{aligned}
$$


Here the linear expressions $\Sigma_{11}^{(1)}, \Sigma_{12}^{(1)}$, and $\mu^{(1)}$ have been given earlier in Chapter 3 [see (3.20) and (3.3.5)]. while the quadratic contributions $\Sigma_{11}^{(2)}$ and $\Sigma_{12}^{(2)}$ can be deduced from $(5.47)$ and $(5.48)$ in the Section 5.2. The "second-order" correction to $\mu$. denoted by $\mu^{(2)}$. can be obtained using Hugenholtz-Pines theorem [23. 26]. namely

$$
\mu^{(2)}=\Sigma_{11}^{(2)}(0)-\Sigma_{12}^{(2)}(0)
$$

With a little algebra. one can show, after dropping all terms of the order $O\left(f_{0}^{4}\right)$ and higher. that the denominator of $G_{11}(p)$ and $C_{12}(p)$ in (2.5:3) and (2.5.t) is given by

$$
D \simeq\left(i \omega_{n}\right)^{2}-i \omega_{n}\left(\Sigma_{11}^{+}-\Sigma_{11}^{-}\right)-E_{k}^{2}+\left(\epsilon_{k}+\Delta\right)\left(2 \mu^{(2)}-\Sigma_{11}^{+}-\Sigma_{11}^{-}\right)+2 \Delta \Sigma_{12}^{(2)}
$$

where

$$
\begin{aligned}
& \Sigma_{11}^{+} \equiv \Sigma_{11}^{(2)}(p) . \\
& \Sigma_{11}^{-} \equiv \Sigma_{11}^{(2)}(-p) .
\end{aligned}
$$

Solving $D\left(\mathbf{k} . i \omega_{n}\right)=0$ for $i \omega_{n}$ (which will be analytically continued to real frequencies 土in). We obtain

$$
\begin{aligned}
i \omega_{n} & \simeq \frac{1}{2}\left(\Sigma_{11}^{+}-\Sigma_{11}^{-}\right) \pm \sqrt{E_{k}^{2}-\left(\epsilon_{k}+\Delta\right)\left(2 \mu^{(2)}-\Sigma_{11}^{+}-\Sigma_{11}^{-}\right)-2 \Delta \overline{\Sigma_{12}^{(2)}}} \\
& \simeq \pm\left\{E_{k}+\frac{1}{2 E_{k}}\left(\epsilon_{k}+\Delta\right)\left(\Sigma_{11}^{+}+\Sigma_{11}^{-}-2 \mu^{(2)}\right)-\frac{1}{E_{k}} \Delta \Sigma_{12}^{(2)} \pm \frac{1}{2}\left(\Sigma_{11}^{+}-\Sigma_{11}^{-}\right)\right\} \\
& \equiv \pm\left(E_{k}+\Lambda_{k}^{ \pm}\right) .
\end{aligned}
$$

Here $i \omega_{n}= \pm E_{k}$ are the two poles of the Green's functions in the first-order Popov approximation [see (3.37)] and the new term in (5.58) is the correction from the second-order B-P approximation,

$$
\Lambda_{k}^{ \pm} \equiv \frac{\epsilon_{k}}{2 E_{k}}\left(\Sigma_{11}^{+}+\Sigma_{11}^{-}-2 \mu^{(2)}\right)+\frac{\Delta}{2 E_{k}}\left(\Sigma_{11}^{+}+\Sigma_{11}^{-}-2 \mu^{(2)}-2 \Sigma_{12}^{(2)}\right) \pm \frac{1}{2}\left(\Sigma_{11}^{+}-\Sigma_{11}^{-}\right)
$$


Using (5.58), the denominator $D\left(\mathbf{k}, i \omega_{n}\right)$ of $G_{11}$ and $G_{12}$ is given by

$$
D\left(\mathbf{k} \cdot i \omega_{n}\right)=\left(i \omega_{n}-E_{k}-\Lambda_{k}^{+}\right)\left(i \omega_{n}+E_{k}+\Lambda_{k}^{-}\right)
$$

Consequently: the diagonal Green's function in the B-P approximation can be rewritten in the form

$$
G_{11}\left(\mathbf{k}, i \omega_{n}\right)=\frac{i \omega_{n}+\epsilon_{k}-\mu^{(1)}-\mu^{(2)}+\Sigma_{11}^{(1)}+\Sigma_{11}^{-}}{\left(i \omega_{n}-E_{k}-._{k}^{+}\right)\left(i \omega_{n}+E_{k}+I_{k}^{-}\right)}
$$

This can be expressed as the sum of two poles.

$$
C_{11}(p)=\frac{A_{k}^{\prime}}{\omega-E_{k}-\Lambda_{k}^{+}}-\frac{B_{k}^{\prime}}{\omega+E_{k}+\Lambda_{k}^{-}} .
$$

where $A_{k}^{\prime}$ and $B_{k}^{\prime}$ satisfy

$$
A_{k}^{\prime}\left(i \omega_{n}+E_{k}+\Lambda_{k}^{-}\right)-B_{k}^{\prime}\left(i \omega_{n}-E_{k}-\Lambda_{k}^{+}\right) \equiv i \omega_{n}+\epsilon_{k}+\Delta-\mu^{(2)}+\Sigma_{11}^{-}
$$

Since (5.63) must hold for arbitrary values of $i \omega_{n}$, we can solve (5.63) for $A_{k}^{\prime}$ and $B_{k}^{\prime}$. to give

$$
\begin{aligned}
& A_{k}^{\prime}=A_{k}+\alpha_{k} . \\
& B_{k}^{\prime}=B_{k}+\alpha_{k} .
\end{aligned}
$$

Here $A_{k}$ and $B_{k}$. defined in (5.22) and (5.23), are the corresponding weights for the two poles $\pm E_{k}$ of $C_{11}$ in the first-order Popov approximation: and $\alpha_{k}$ is the "secondorder" correction defined by

$$
\alpha_{k} \equiv \frac{\Delta}{4 E_{k}^{3}}\left[2 \epsilon_{k} \Sigma_{12}^{(2)}-\Delta\left(\Sigma_{11}^{+}+\Sigma_{11}^{-}-2 \mu^{(2)}-2 \Sigma_{12}^{(2)}\right)\right]
$$

Using (5.64) and (5.65) in (5.62), we finally arrive at

$$
G_{11}\left(k \cdot i \omega_{n}\right)=\frac{A_{k}+\alpha_{k}}{i \omega_{n}-E_{k}-\Lambda_{k}^{+}}-\frac{B_{k}+\alpha_{k}}{i \omega_{n}+E_{k}+\Lambda_{k}^{-}}
$$


This type of expression was first derived by Beliaev [34] at $T=0$ and the above analysis formally extends his approach to finite temperature.

Following the analysis in obtaining ( 5.67$)$, it is straightforward to also express the anomalous Green's function $G_{12}$ in a similar way. namely

$$
G_{12}\left(\mathbf{k} \cdot i \omega_{n}\right)=\frac{C_{k}+\gamma_{k}}{i \omega_{n}-E_{k}-\Lambda_{k}^{+}}-\frac{C_{k}+\gamma_{k}}{i \omega_{n}+E_{k}+\Lambda_{k}^{-}}
$$

Here the "second-order" correction to $C_{k}$ is given by

$$
\gamma_{i k} \equiv \frac{\Delta}{2 E_{k}^{3}}\left[\epsilon_{k}\left(\Sigma_{11}^{+}+\Sigma_{11}^{-}-2 \mu^{(2)}\right)+\Delta\left(\Sigma_{11}^{+}+\Sigma_{12}^{-}-2 \mu^{(2)}-2 \Sigma_{12}^{(2)}\right)\right]-\frac{\Sigma_{12}^{(2)}}{2 E_{k}}
$$

We can use (5.67) and (5.68) to calculate the properties of the Green's function within the B-P approximation. This will be done in Chapter 6 and $\tau$. The energy of single-particle excitations (poles of $C_{11}$ and $C_{12}$ ) are given by

$$
E_{k}^{B P}= \pm\left(E_{k}+. \Lambda_{k}^{ \pm}\right)
$$

which may contain a finite imaginary part. describing the damping of the singleparticle excitations. We emphasis that these approximate expressions for $G_{11}$. $C_{12}$ and $E_{k}^{B P}$ are only valid within the B-P approximation. They can not used in other approximations without further discussion. 


\section{Chapter 6}

\section{Calculations at $T=0$ using the B-P approximation}

In Chapter 5. we worked out the single-particle Green's functions in the B-P approximation for a weakly-interacting Bose gas. The formalism developed was valid for arbitrary temperature. In this chapter. we use the results obtained in Chapter 5 to study the special case of $T=0$. reproducing the famous "second-order" calculations worked out by Beliaev [3:4] some forty years ago. We have tried to give a more detailed treatment of some of the intermediate steps and hope that our discussion will be useful to many readers who have become interested in Beliaev's work in connection with calculating the properties of the recently discovered trapped atomic Bose gases. In particular, we give. for the first time. explicit expressions for the self-energies in the B-P approximation which contain infrared divergent terms. As well-known [22. 30]. these divergent contributions cancel out in the final expressions for physical quantities. such as the long-wavelength excitation spectrum. However, they have a physical basis in the Bose broken symmetry and are of interest in this more general context. 


\subsection{The B-P self-energies at $T=0$}

At zero temperature. the diagonal Green's function $C_{t_{1}}$ in $(5.6 \tau)$ is given by

$$
G_{11}(\mathbf{k} . \omega)=\frac{A_{k}+\alpha_{k}}{\omega-E_{k}-\Lambda_{k}^{+}+i \eta}-\frac{B_{k}+\alpha_{k}}{\omega+E_{k}+\Lambda_{k}^{-}-i \eta} .
$$

where $\Lambda_{k}^{ \pm}$and $\alpha_{k}$ are defined in (5.59) and (5.66). The functions $\alpha_{k}$ and $\Lambda_{k}^{ \pm}$are combinations of $\Sigma_{11}^{(2)}( \pm p)$ (or $\left.\Sigma_{11}^{ \pm}\right), \Sigma_{12}^{(2)}$ and $\mu^{(2)}$. which are "second-order" corrections to the corresponding quantity $\Sigma_{11}( \pm p) . S_{12}(p)$, and $\mu$ respectively. Csing (5.49) and $(5.50)$. leaving out the first-order terms and carrying out the following integrals.

$$
\begin{array}{r}
2 \dot{f}_{0} \int \frac{d \mathbf{k}_{1}}{(2 \pi)^{3}} B_{1}=2 \tilde{f}_{0} \int \frac{d \mathbf{k}_{1}}{(2 \pi)^{3}} \frac{-E_{1}+\epsilon_{1}+\Delta}{2 E_{1}}=\frac{2}{3 \pi^{2}}\left(\frac{m}{\hbar^{2}}\right)^{3 / 2} \sqrt{n_{0} f_{0}^{3}} \Delta \\
n_{0} \dot{f}_{0}^{2} \int \frac{d \mathbf{k}_{1}}{(2 \pi)^{3}}\left(\frac{\mathcal{P}}{\epsilon_{1}-\epsilon_{k / 2}}-\frac{1}{E_{1}}\right)=\frac{2}{\pi^{2}}\left(\frac{m \Delta}{\hbar^{2}}\right)^{3 / 2} \sqrt{n_{0} \dot{f}_{0}^{3}} \Delta .
\end{array}
$$

we obtain the $T=0$ "second-order" terms

$$
\begin{aligned}
\Sigma_{11}^{(2)}(p)= & \frac{1}{2} n_{0} \dot{f}_{0}^{2} \int \frac{d \mathbf{k}_{1}}{(2 \pi)^{3}} \frac{1}{E_{1} E_{2}}\left[\frac{g\left(E_{1}, E_{2}\right)}{\omega-E_{1}-E_{2}+i \eta}-\frac{g\left(-E_{1},-E_{2}\right)}{\omega+E_{1}+E_{2}-i \eta}\right. \\
& \left.+E_{1}+E_{2}\right]+\frac{s}{3 \pi^{2}}\left(\frac{m}{\hbar^{2}}\right)^{3 / 2} \sqrt{n_{0} \dot{f}_{0}^{3}} \lambda . \\
\Sigma_{12}^{(2)}(p)= & \frac{1}{2} n_{0} \dot{f}_{0}^{2} \int \frac{d \mathbf{k}_{1}}{(2 \pi)^{3}} \frac{1}{E_{1} E_{2}}\left[\frac{h\left(E_{1} \cdot E_{2}\right)}{\omega-E_{1}-E_{2}+i \eta}-\frac{h\left(-E_{1}-E_{2}\right)}{\omega+E_{1}+E_{2}-i \eta}\right] \\
& +\frac{1}{\pi^{2}}\left(\frac{m}{\hbar^{2}}\right)^{3 / 2} \sqrt{n_{0} \dot{f}_{0}^{3}} \lambda .
\end{aligned}
$$

Here the functions $g$ and $h$ are defined by $(5.30)$ and $(5.34)$, respectively: and $\lrcorner \equiv n_{0} \tilde{f}_{0}$ [see (3.36)]. The results in (6.3) and (6.4) agree precisely with those given by Eqs.(5.9) and (5.10) of Beliaev [34].

The form of (6.1) makes it convenient to study the behavior of the Green s function near the poles at $\omega \simeq \pm E_{k}$. For this purpose, we first do a Taylor expansion of $\Sigma_{11}^{(2)}(\mathbf{k}, \omega)$ and $\Sigma_{12}^{(2)}(\mathbf{k}, \omega)$ about the pole $\omega=E_{k}$, 


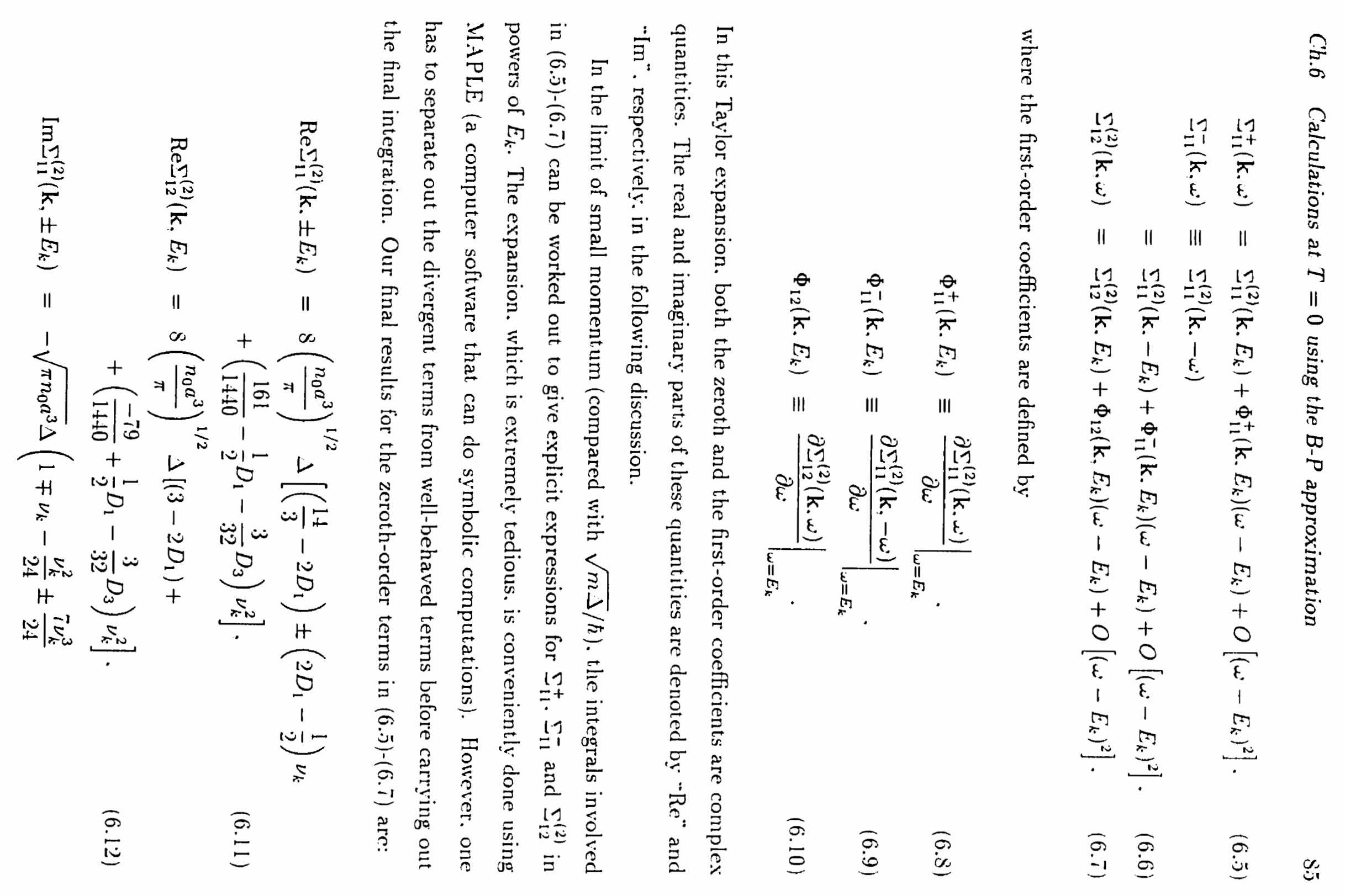




$$
\begin{gathered}
\left.+\frac{13 \nu_{k}^{4}}{1920} \mp \frac{193 \nu_{k}^{5}}{1920}+\frac{30 \pi \nu_{k}^{6}}{35840}\right) . \\
\left.\operatorname{Im} \Sigma_{12}^{(2)}\left(\text { k. } E_{k}\right)=-\sqrt{\pi n_{0} a^{3}}\right\lrcorner\left(1-\frac{13 \nu_{k}^{2}}{24}+\frac{293 \nu_{k}^{4}}{1920}-\frac{125.5 \nu_{k}^{6}}{21.504}\right) .
\end{gathered}
$$

Here $a$ (from $\tilde{f}_{0}=4 \pi \hbar^{2} a / m$ ) is the s-wave scattering length: the quantity $\left(n_{0} a^{3}\right)^{1 / 2}$ is a small dimensionless parameter in a low density gas [see (3.7)]. The dimensionless ratio $\nu_{k}$ defined as

$$
\nu_{k} \equiv \frac{E_{k}}{J}
$$

is also a small quantity in the limit of $k \ll \sqrt{m \Delta} / \hbar$. The other two quantities $D_{1}$ and $D_{3}$ are dimensionless integrals:

$$
\begin{aligned}
D_{1} & \equiv \int_{0}^{\infty} \frac{d x}{x\left(x^{2}+4\right)^{3 / 2}} \\
D_{3} & \equiv \int_{0}^{\infty} \frac{d x}{x^{3}\left(x^{2}+4\right)^{\tilde{T} / 2}}
\end{aligned}
$$

One sees that as $x \rightarrow 0$. both integrals $D_{1}$ and $D_{3}$ are divergent: $D_{1}$ goes to infinity as $\ln x$ and $D_{3}$ as $x^{-2}$. which shows the infrared divergence inherent in the quantum field analysis of a Bose-condensed system [2.2, 30]. As far as we know. this is the first time that infrared divergence associated with these higher-order self-energy expressions have been written down explicitly. Comparing (6.11) and (6.12) for the rea! parts $\operatorname{Re} \Sigma_{11}^{(2)}$ and $\operatorname{Re} \Sigma_{12}^{(2)}$ with those given by Cheung and Griffin [29!. we note that our coefficient of the $\nu_{k}^{2}$ term is different, and furthermore the infrared divergent terms were left out of their results.

Similarly, coefficients of the linear terms in the Taylor expansion of $\Sigma_{11}^{ \pm}(\mathbf{k} . \omega)$ and $S_{\mathrm{t} 2}^{(2)}(\mathbf{k} . \omega)$ in (6.5)-(6.7) can be obtained by (a) taking their derivatives with respect to $\omega$. (b) evaluating the derivatives at $\omega=E_{k}$. and (c) in the limit of small $k$. expanding the integrands in powers of $E_{k}$. This procedure gives (again with the aid of MAPLE)

$$
\operatorname{Re} \Phi_{11}^{ \pm}\left(\mathbf{k}, E_{k}\right)=\delta\left(\frac{n_{0} a^{3}}{\pi}\right)^{1 / 2}\left[\mp\left(\frac{1}{2}-2 D_{1}\right)-\left(\frac{1}{15}+\frac{1}{4} D_{1}+16 D_{3}\right) \nu_{k}\right]
$$




$$
\begin{aligned}
& \operatorname{Re} \Phi_{12}\left(\mathrm{k} . E_{k}\right)=8\left(\frac{n_{0} a^{3}}{\pi}\right)^{1 / 2}\left(\frac{\pi}{4} D_{1}-16 D_{3}-\frac{1}{15}\right) \nu_{k} \\
& \operatorname{Im} \Phi_{11}^{ \pm}\left(\mathrm{k} . E_{k}\right)=-\sqrt{\pi n_{0} a^{3}}\left(\mp 1-\frac{\nu_{k}}{2} \pm \frac{25 \nu_{k}^{2}}{24}+\frac{13 \nu_{k}^{3}}{48} \mp \frac{11333 \nu^{4}}{19 \cdot 20}-\frac{97 \nu_{k}^{5}}{168}\right) \\
& \operatorname{Im} \Phi_{12}\left(\mathrm{k} . E_{k}\right)=-\sqrt{\pi n_{0} a^{3}}\left(-\frac{3 \nu_{k}}{2}+\frac{13 \nu_{k}^{3}}{16}-\frac{613 \nu_{k}^{5}}{1280}\right) .
\end{aligned}
$$

Here the imaginary terms have to be evaluated up to order $E_{k}^{5}$ because the lower-order terms all cancel out when we calculate the quasiparticle energy spectrum.

\subsection{Quasiparticle spectrum}

In the previous section. we have obtained all the zero-order and first-order terms in the Taylor expansion of $\Sigma_{11}^{ \pm}$and $\Sigma_{12}^{(2)}$ around the pole $\omega=E_{k}$. The "second-order" correction to the chemical potential at $T=0$ can now be easily calculated using $(5.54)$ and $(6.11)-(6.14)$. to give

$$
\left.\mu^{(2)}=\frac{40}{3}\left(\frac{n_{0} a^{3}}{\pi}\right)^{1 / 2}\right\lrcorner
$$

With these results for $\Sigma_{11}^{ \pm} . \Sigma_{12}^{(2)}$. and $\mu^{(2)}$. it is straightforward to calculate $\alpha_{k}$ and .$\Lambda_{k}^{+}$. defined by (5.66) and (5.59). We obtain

$$
\begin{aligned}
\alpha_{k}= & \left(\frac{n_{0} a^{3}}{\pi}\right)^{1 / 2}\left(\frac{16}{3} \frac{\partial}{E_{k}}+i \frac{\pi}{8} \frac{E_{k}}{\Delta}\right) . \\
\Lambda_{k}^{+}= & \left(\frac{n_{0} a^{3}}{\pi}\right)^{1 / 2}\left(\frac{28}{3} E_{k}-i \frac{3 \pi}{80} \frac{E_{k}^{5}}{\Delta^{4}}\right) \\
& -\left(\frac{n_{0} a^{3}}{\pi}\right)^{1 / 2}\left(4+i \frac{\pi}{4} \frac{E_{k}^{2}}{\Delta^{2}}\right)\left(\omega-E_{k}\right) \\
\equiv & \Xi_{k}-\lambda_{k}\left(\omega-E_{k}\right),
\end{aligned}
$$

where (6.24) also defines the new functions $\Xi_{k}$ and $\lambda_{k}$.

The calculation of $\Lambda_{k}^{-}$requires expansions of $\Sigma_{11}^{ \pm}$and $\Sigma_{12}^{(2)}$ near the negative fre- 
quency pole $\omega=-E_{k}$. Using the following equality for any differentiable function $y(x)$ of $x:$

$$
\left.\frac{d y(-x)}{d x}\right|_{x=x_{0}}=-\left.\frac{d y(x)}{d x}\right|_{x=-x_{0}}
$$

we have

$$
\begin{aligned}
& \Phi_{11}^{+}\left(\mathbf{k} \cdot-E_{k}\right)=-\Phi_{11}^{-}\left(\mathbf{k} \cdot E_{k}\right) \\
& \Phi_{11}^{-}\left(\mathbf{k} \cdot-E_{k}\right)=-\Phi_{11}^{+}\left(\mathbf{k} \cdot E_{k}\right), \\
& \Phi_{12}\left(\mathbf{k} \cdot-E_{k}\right)=-\Phi_{12}\left(\mathbf{k} \cdot E_{k}\right) .
\end{aligned}
$$

With these last three equalities. we can calculate the quantity $\lambda_{k}^{-}$near the negative pole $\omega^{\prime}=-E_{k}$ to obtain

$$
\begin{aligned}
I_{k}^{-}= & \left(\frac{n_{0} a^{3}}{\pi}\right)^{1 / 2}\left(\frac{28}{3} E_{k}-i \frac{3 \pi}{80} \frac{E_{k}^{5}}{\Delta^{4}}\right) \\
& +\left(\frac{n_{0} a^{3}}{\pi}\right)^{1 / 2}\left(4+i \frac{\pi}{4} \frac{E_{k}^{2}}{\Delta^{2}}\right)\left(\omega+E_{k}\right) \\
\equiv & \Xi_{k}+\lambda_{k}\left(\omega+E_{k}\right) .
\end{aligned}
$$

The results of $(6.23) .(6.24)$ and (6.29) were first obtained by Beliacs in [3-4]. if we recall that in his notation $a=f_{0} / 4 \pi$. and $\Delta=n_{0} f_{0}$. However, as we have noted earlier. Beliaev did not write down the equivalent of (6.11)-(6.14) and (6.1S)-(6.21). nor did he mention the infrared divergent terms.

With (6.24). (6.29) and (6.1). the Green's function near to the poles at small momenta may be written in the form

$$
C_{\mathrm{Il}}(\mathrm{k}, \omega)=\left(\mathrm{l}-\lambda_{k}\right)\left[\frac{A_{k}+\alpha_{k}}{\omega-E_{k}-\Xi_{k}}-\frac{B_{k}+\alpha_{k}}{\omega+E_{k}+\Xi_{k}}\right]
$$

Comparison with the first-order diagonal Green's function given by (3.31). one sees that the quantities $\lambda_{k}, \alpha_{k}$ and $\Xi_{k}$ are "second-order" corrections. These corrections are small for a low-density gas, since they are all proportional to $\sqrt{n_{0} a^{3}}<\sqrt{n a^{3}} \ll \mathrm{I}$ under the condition (3.T). 
The quasiparticle energy is determined by the pole of $G_{1_{1}}(\mathbf{k} . \omega)$. In the momentum range $|\mathbf{k}| \ll \sqrt{m \Delta} / \hbar .(6.1)$ and $(6.2 t)$ together yield

$$
\begin{aligned}
E_{k}^{(2)} & =E_{k}+\Xi_{k} \\
& =E_{k}\left[1+\frac{28}{3}\left(\frac{n_{0} a^{3}}{\pi}\right)^{1 / 2}\right]-i \frac{3}{80} \sqrt{\pi n_{0} a^{3}} \frac{E_{k}^{5}}{\Delta^{4}}
\end{aligned}
$$

We recall that $E_{k}=\hbar c k$ for small momenta $k \ll \sqrt{m \Delta} / \hbar$. where $c=\sqrt{\Delta / m}=$ $\hbar \sqrt{4 \pi n_{0} a} / m$ is the sound velocity in the first-order approximation. Eq.(6.31) shows that for small momentum, the quasiparticles are phonon-like. The "second-order" approximation gives a small correction to the sound velocity

$$
c^{(2)}=c\left[1+\frac{2 S}{3}\left(\frac{n_{0} a^{3}}{\pi}\right)^{1 / 2}\right]
$$

In addition. $E_{k}^{(2)}$ contains an imaginary part proportional to $E_{k}^{5}$ or $k^{5}$. This corresponds to a finite lifetime for the phonons. This $T=0$ long wavelength damping is due to the decay of a phonon into two single excitations. This is clear from the structure of the self-energies in $(6.3)$ and $(6.4)$ with poles at $\omega= \pm\left(E_{1}+E_{2}\right)$.

The mean number of the atoms $\bar{V}_{k}$ with a given momentum $k$ at $T=0$ can be calculated directly from the Green's function. using

$$
\begin{aligned}
\bar{v}_{k} & =i \lim _{\eta \rightarrow 0^{+}} \int_{-\infty}^{+\infty} \frac{d \omega}{2 \pi} e^{i \omega \eta} G_{11}(\text { k. } \omega) \\
& =-\oint_{C} \frac{d z}{2 \pi i} \epsilon^{i z \eta}\left(1-\lambda_{k}\right)\left[\frac{A_{k}+\alpha_{k}}{z-E_{k}-\Xi_{k}}-\frac{B_{k}+\alpha_{k}}{z+E_{k}+\Xi_{k}}\right]
\end{aligned}
$$

Here the path of integration $C$ is a contour consisting of the real axis from $-\infty$ to $+\infty$. together with a semicircle in the upper half plane. We emphasize that $\bar{V}_{k}$ is the momentum distribution of atoms, not the quasiparticle momentum distribution. Because the imaginary part of $\Xi_{k}$ is negative [see(6.24)], the only contribution is from the negative energy pole of $G_{11}$ at $\omega=-E_{k}-\Xi_{k}$. Therefore using (5.2:3) and (6.33). 
we obtain

$$
\begin{aligned}
\bar{N}_{k} & =\left(1-\lambda_{k}\right)\left(B_{k}+\alpha_{k}\right) \\
& =\frac{\Delta}{2 E_{k}}\left[1+\frac{20}{3}\left(\frac{n_{0} a^{3}}{\pi}\right)^{1 / 2}\right] .
\end{aligned}
$$

The imaginary parts of $\alpha_{k}$ and $\lambda_{k}$ cancel in (6.34). and thus $\bar{N}_{k}$ is real. as it should be. To calculate the total number of particles with non-zero momentum. we need to know $\bar{V}_{k}$ for all momenta. Unfortunately, no general analytic form is arailable for arbitrary momentum. Numerical methods are required to calculate the number of particles $\dot{V}$ with non-zero momentum. from which, one can find the number $\dot{V}_{0}$ of atoms in the condensate. for a fixed total number of atoms.$V$.

We can also calculate the ground-state energy from the chemical potential $\mu$. The first-order contribution to $\mu$ is $\mu^{(1)}=\Delta=n_{0} \tilde{f}_{0}$ given by (3.3.5) and the "second-order" contribution $\mu^{(2)}$ is given by (6.22). We add these two parts to obtain

$$
\mu=\frac{4 \pi \hbar^{2} n_{0} a}{m}\left[1+\frac{40}{3}\left(\frac{n_{0} a^{3}}{\pi}\right)^{1 / 2}\right] .
$$

Here we may use the result for $n_{0}$ obtained in the first-order Popov approximation. as given by (3.4t) of Chapter 3. Expressing $n_{0}$ in terms of $n$ by means of (3.4. ). we finally obtain

$$
\mu=\frac{4 \pi \hbar^{2} n a}{m}\left[1+\frac{32}{3}\left(\frac{n a^{3}}{\pi}\right)^{1 / 2}\right] .
$$

$\mathrm{By}$ definition, at $T=0$ we have $\mu=\partial\left(E_{0} / V\right) / \partial n$. Therefore. integrating (6.36) with respect to $n$. one obtains the ground-state energy

$$
\frac{E_{0}}{V}=\frac{2 \pi \hbar^{2} n^{2} a}{m}\left[1+\frac{128}{15}\left(\frac{n a^{3}}{\pi}\right)^{1 / 2}\right] .
$$

These result coincides with the result of Lee. Huang and Yang [24] for a hard-sphere gas. and later re-derived by Beliaev [34] and by Hugenholtz and Pines [2:3]. 
The pressure $P$ is given by

$$
P=-\left(\frac{\partial E_{0}}{\partial V}\right)_{x}=\frac{2 \pi \hbar^{2} n^{2} a}{m}\left[1+\frac{64}{5}\left(\frac{n a^{3}}{\pi}\right)^{1 / 2}\right]
$$

The derivative of $P$ with respect to $n$ yields the usual compressional speed of sound

$$
\begin{aligned}
s=\left(\frac{1}{m} \frac{\partial P}{\partial n}\right)^{1 / 2} & =\left(\frac{4 \pi \hbar^{2} n a}{m}\right)^{1 / 2}\left[1+8\left(\frac{n a^{3}}{\pi}\right)^{1 / 2}\right] \\
& =\left(\frac{4 \pi \hbar^{2} n_{0} a}{m}\right)^{1 / 2}\left[1+\frac{28}{3}\left(\frac{n_{0} a^{3}}{\pi}\right)^{1 / 2}\right] .
\end{aligned}
$$

Comparison of $(6.39)$ with $(6.32)$ shows that the phonon velocity $c^{(2)}$ is equal to the compressional speed of sound $s$ in the second-order approximation. This is in agreement with Gavoret and Nozières's famous result that, to arbitrary order in the perturbation. the phonon velocity equals the macroscopic speed of sound at $T=0$.

We now turn to the calculation of the spectral density function $A(k . w)$. which we recall from (hapter 2 is given by

$$
A(k \cdot \omega)=-2 \operatorname{Im} G_{11}^{\prime}(k \cdot \omega)
$$

At the end of the previous section. we obtained an expression for the Green s function $C_{11}($ k. $\omega)$. Using the results for $\alpha_{k}, \lambda_{k}$ and $\Xi_{k}$ in $(6.23)$ and $(6.24)$. we find after some calculation that the spectral density function is given by $\left(\nu_{k} \equiv E_{k} / \Delta=\hbar c k / \triangle\right.$ : $\left.\Delta \equiv n_{0}+\pi \hbar^{2} a / m\right)$

$$
\begin{aligned}
A(\mathbf{k} . \omega)= & \frac{\pi^{1 / 2}}{80}\left(n_{0} a^{3}\right)^{1 / 2} \nu_{k}^{2}\left[\frac{\omega\left(20+10 \nu_{k}\right)-E_{k}\left(20+\pi \nu_{k}\right)}{\left(\omega-E_{k}\right)^{2}+\gamma_{k}^{2}}\right. \\
& \left.-\frac{\omega\left(-20+10 \nu_{k}\right)-E_{k}\left(20-i \nu_{k}\right)}{\left(\omega+E_{k}\right)^{2}+\gamma_{k}^{2}}\right] \\
\dot{E}_{k} & \equiv \hbar c k\left[1+\frac{2 S}{3}\left(\frac{n_{0} a^{3}}{\pi}\right)^{1 / 2}\right] \\
\gamma_{k} & =\frac{3 \pi}{80}\left(\frac{n_{0} a^{3}}{\pi}\right)^{1 / 2} \frac{(\hbar c k)^{5}}{\Delta^{4}}=\frac{3}{640 \pi} \frac{\hbar^{2}}{m}\left(\frac{k^{5}}{n_{0}}\right) .
\end{aligned}
$$


Somewhat surprisingly. $\gamma_{k}$ in (6.t3) does not explicitly depend on the parameter $a$ (except for an implicit, weak dependence through $n_{0}$. the condensate density). One recalls that the collision rate goes as $a^{2}$ for a dilute classical gas. The result in (6.t3). therefore. is puzzling at first glance. It is a consequence of the fact that at $T=0$. the quasiparticles are phonons rather than free atoms. The excitation energy depends on $\sqrt{a}$ in the long wavelength limit. and therefore the s-wave scattering length $a$ enters the calculation of one phonon decaying into two in a complicated way. The final result is that $a$ cancels out in the expression for $\%$ in $(6.43)$.

The explicit expression given in $(6.41)$ has not been given in the previous literature. We recall that in the Bogoliubov approximation, the spectral density function is given by

$$
A(\mathbf{k} \cdot \omega)=A_{k} \delta\left(\omega-E_{k}\right)-B_{k} \delta\left(\omega+E_{k}\right),
$$

which consists of two peaks at $E_{k}$ and $-E_{k}$ respectively. Associated with the damping of the excitations, the peaks in $(6.41)$ have a finite width.

It is easy to check that at $\omega=0 .(6 .+1)$ reduces to

$$
A(\mathrm{k} \cdot \omega=0) \simeq-\frac{i \pi^{1 / 2}}{40}\left(n a^{3}\right)^{1 / 2} \frac{E_{k}^{2}}{\lrcorner^{3}}
$$

for small $k\left(E_{k} \ll \Delta\right)$. By definition. $A(\mathrm{k} . \omega=0)$ should vanish for any value of $\mathrm{k}$ [see Eq.(2.60)]. However, if we remember the Green's function $C_{11}(\mathbf{k} . \omega)$ in $(6.30)$ is only valid at near the poles $w \sim \pm E_{k}$. the small but finite zero frequency value in $(6.45)$ is not unexpected. 


\section{Chapter 7}

\section{Discussion of the $T \neq 0$ B-P}

\section{approximation}

In this chapter, we use the self-energies obtained in $(5.47)$ and $(5.48)$ in Chapter 5 to consider the case of finite temperature. These self-energies can be separated into two parts: one part denoted by $\Sigma^{0}$ which does not depend explicitly on the Bose factor: and another temperature-dependent part denoted by $\Sigma^{T}$ which does depend on the Bose factor. In Chapter 6. we worked out the expressions for $\Sigma^{0}$ near the poles

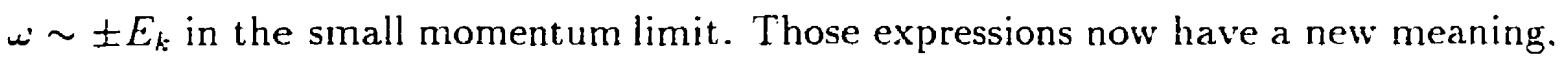
since they involve the condensate density $n_{0}(T)$ which is temperature-dependent at finite temperature.

We now turn our attention to the temperature dependent parts of the self-energies associated with the Bose factors. namely

$$
\begin{aligned}
\Sigma_{11}^{T}(p)= & n_{0} \dot{f}_{0}^{2} \int \frac{d \mathbf{k}_{1}}{(2 \pi)^{3}} \frac{1}{E_{1} E_{2}}\left[\frac{g\left(E_{1}, E_{2}\right)}{\omega-E_{1}-E_{2}+i \eta}-\frac{g\left(-E_{1},-E_{2}\right)}{\omega+E_{1}+E_{2}+i \eta}\right. \\
& \left.+\frac{g\left(-E_{1}, E_{1}\right)}{\omega+E_{1}-E_{2}+i \eta}-\frac{g\left(E_{1},-E_{2}\right)}{\omega-E_{1}+E_{2}+i \eta}\right] f_{B}\left(E_{1}\right) \\
& +2 \tilde{f}_{0} \int \frac{d \mathbf{k}_{1}}{(2 \pi)^{3}} \frac{\epsilon_{1}+\Delta}{E_{1}} f_{B}\left(E_{1}\right) \\
\Sigma_{12}^{T}(p)= & n_{0} \tilde{f}_{0}^{2} \int \frac{d \mathbf{k}_{1}}{(2 \pi)^{3}} \frac{1}{E_{1} E_{2}}\left[\frac{h\left(E_{1}, E_{2}\right)}{\omega-E_{1}-E_{2}+i \eta}-\frac{h\left(-E_{1},-E_{2}\right)}{\omega+E_{1}+E_{2}+i \eta}\right.
\end{aligned}
$$




$$
\begin{aligned}
& \left.+\frac{h\left(-E_{1}, E_{1}\right)}{\omega+E_{1}-E_{2}+i \eta}-\frac{h\left(E_{1},-E_{2}\right)}{\omega-E_{1}+E_{2}+i \eta}\right] f_{B}\left(E_{1}\right) \\
& -n_{0} f_{0}^{2} \int \frac{d \mathbf{k}_{1}}{(2 \pi)^{3}} \frac{1}{E_{1}} f_{B}\left(E_{1}\right) .
\end{aligned}
$$

Here we remind the reader that the functions $g$ and $h$ are as defined in (5.30) and $(5.3+1)$. respectively:

$$
\begin{aligned}
& g\left(E_{1}, E_{2}\right)=3 \epsilon_{1} \epsilon_{2}-E_{1} E_{2}+\Delta\left(\epsilon_{1}+\epsilon_{2}\right)+\Delta^{2}-\Delta\left(E_{1}+E_{2}\right)+\epsilon_{1} E_{2}+\epsilon_{2} E_{1} \\
& h\left(E_{1} \cdot E_{2}\right)=2 \epsilon_{1} \epsilon_{2}-2 E_{1} E_{2}+\Delta^{2} .
\end{aligned}
$$

and the subscript " $l "$ refers to $k_{1}$ and " $2 "$ refers to $k-k_{1}$. We have made the analytical continuation $i \omega_{n} \rightarrow \omega+i \eta$ in the expressions of $\Sigma_{11}^{T}$ and $\Sigma_{12}^{T}$. implying that we are now dealing with the self-energies for the retarded Green's function $C_{r}{ }^{r e t}$ (see Section 2.5).

We recall that at $T=0$. the self-energies in (6.3) and $(6.4)$ involve poles at $\omega= \pm\left(E_{1}+E_{2}\right)$. which describes the decay of one phonon into two phonons. At finite temperature, the self-energies in ( $T .1)$ and $(T .2)$ involve additional poles at $\dot{\nu}= \pm\left(E_{1}-E_{2}\right)$. representing the process of a phonon scattering with a thermallyexcited phonon. Due to these additional thermal scattering processes (present only at finite temperatures). it is difficult to carry out the equivalent calculations to those in Chapter 6. One difficulty is associated with the fact that it now matters what order one takes the limits $\omega \rightarrow 0$ and $k \rightarrow 0$. For example, if one takes the limit $\omega \rightarrow 0$ first and then the limit $\mathbf{k} \rightarrow 0$, one immediately sees that the thermal terms of the diagonal self-energy $\Sigma_{11}^{T}$ in $(T .1)$ involving poles at $\omega= \pm\left(E_{1}-E_{2}\right)$ are singular:

$$
\begin{aligned}
\lim _{\mathrm{k} \rightarrow 0}\left\{\lim _{\omega \rightarrow 0}\left[\frac{g\left(-E_{1}, E_{2}\right)}{\omega+E_{1}-E_{2}}-\frac{g\left(E_{1}-E_{2}\right)}{\omega-E_{1}+E_{2}}\right]\right\} \\
\quad=\lim _{2 \rightarrow 1} \frac{g\left(-E_{1}, E_{2}\right)+g\left(E_{1},-E_{2}\right)}{E_{1}-E_{2}} \\
\quad=\lim _{2 \rightarrow 1} \frac{6 \epsilon_{1} \epsilon_{2}+2 E_{1} E_{2}+2 \Delta\left(\epsilon_{1}+\epsilon_{2}\right)+2 \Delta^{2}}{E_{1}-E_{2}} \\
=\infty
\end{aligned}
$$


The limit of $\mathbf{k} \rightarrow 0$ means $\mathrm{k}_{2}\left(=\mathbf{k}-\mathrm{k}_{1}\right) \rightarrow-\mathrm{k}_{\mathrm{I}}$ [which is equivalent to $\mathrm{k}_{1}$ because the integrands of $(\tau .1)$ and $(\tau .2)$ depends only on $\left|k_{1}\right|$ and $\left.\left|k_{2}\right|\right]$. This is denoted by $\cdots{ }^{*} \rightarrow " 1 "$. Since the numerator of $(7.3)$ is finite. we see the expression is singular for any value of $k_{1}$. On the other hand, if we reverse the order of the two limits in $(7.3)$, we find

$$
\begin{aligned}
\lim _{\omega \rightarrow 0}\left\{\lim _{2 \rightarrow 1}\left[\frac{g\left(-E_{1}, E_{2}\right)}{\omega+E_{1}-E_{2}}-\frac{g\left(E_{1},-E_{2}\right)}{\omega-E_{1}+E_{2}}\right]\right\} \\
\quad=\lim _{\nu \rightarrow 0} \frac{g\left(-E_{1}, E_{1}\right)-g\left(E_{1},-E_{1}\right)}{\omega} \\
=0 .
\end{aligned}
$$

The above limit vanishes because $g\left(-E_{1}, E_{1}\right)=g\left(E_{1},-E_{1}\right)$. The similar kind of analysis also applies to the thermal terms of $\Sigma_{12}^{T}$ in $(7.2)$. Due to this dependence on the order of limits. we can not expand $\Sigma^{T}\left(\mathbf{k}, E_{k}\right)$ in the small momentum limit following the procedure used in Chapter 6 , which effectively took the limits $\rightarrow \rightarrow 0$. $k \rightarrow 0$ at the same time. This kind of dependence on the ratio $k / \omega$ in the limit of $(\mathbf{k}, \omega) \rightarrow 0$ is well-known in the theory of Fermi liquids (see, for example. [46]).

\subsection{The $\mathrm{k}=0$ limit}

We now take the limit $\mathbf{k} \rightarrow 0$ first and then the limit $\omega \rightarrow 0$. In this case. the thermal terms in $S_{11}^{T}$ and $S_{12}^{T}$ vanish. Using (5.4T) and (5.48) for the expressions of the total self-energies $\Sigma_{11}(p)$ and $\Sigma_{12}(p)$ we find that in the above limit. these expressions can be simplified to

$$
\begin{aligned}
\Sigma_{11}(0,0)= & 2 n_{0} \tilde{f}_{0}+2 \tilde{f}_{0} \int \frac{d \mathbf{k}_{\mathrm{l}}}{(2 \pi)^{3}}\left(\frac{\epsilon_{1}+\Delta}{2 E_{1}} \operatorname{coth} \frac{3 E_{1}}{2}-\frac{1}{2}\right) \\
& -n_{0} \tilde{f}_{0}^{2} \int \frac{d \mathbf{k}_{1}}{(2 \pi)^{3}} \frac{1}{E_{1}}\left[\left(1-\frac{2 \Delta \epsilon_{1}}{E_{1}^{2}}+\frac{\Delta^{2}}{2 E_{1}^{2}}\right) \operatorname{coth} \frac{3 E_{1}}{2}-\frac{1}{\epsilon_{1}}\right] . \\
\Sigma_{12}(0,0)= & n_{0} \tilde{f}_{0}-n_{0} \tilde{f}_{0}^{2} \int \frac{d \mathbf{k}_{1}}{(2 \pi)^{3}} \frac{1}{E_{1}}\left[\left(\frac{1}{2}-\frac{2 \Delta \epsilon_{1}}{E_{1}^{2}}+\frac{\Delta^{2}}{2 E_{1}^{2}}\right) \operatorname{coth} \frac{3 E_{1}}{2}-\frac{1}{2 \epsilon_{1}}\right] .
\end{aligned}
$$


The second term on the right hand side of (T.5) equals the density $\dot{n}^{(1)}$ of noncondensate atoms in the first approximation, given by (3.42). It is very important to note that $n_{0}$ which appears in these results is the temperature-dependent condensate $n_{0}(T)$. to be calculated self-consistently. We note that both $\Sigma_{11}(0.0)$ and $\Sigma_{12}(0.0)$ contain the term

$$
-n_{0} \tilde{f}_{0}^{2} \int \frac{d \mathbf{k}_{1}}{(2 \pi)^{3}} \frac{\lrcorner^{2}}{E_{1}^{3}} \operatorname{coth} \frac{3 E_{1}}{2} .
$$

which is divergent at $k_{1} \rightarrow 0$. namely

$$
\int d \mathbf{k}_{1} \frac{1}{E_{1}^{3}} \operatorname{coth} \frac{3 E_{1}}{2} \times \int d k_{1} \frac{k_{1}^{2}}{E_{1}^{4}} \times \int \frac{d k_{1}}{k_{1}^{2}} .
$$

The chemical potential for the condensed phase can be calculated using the Hugenholtz-Pines theorem:

$$
\begin{aligned}
\mu & =\Sigma_{11}(0,0)-\Sigma_{12}(0.0) \\
& =n_{0} \tilde{f}_{0}+2 \tilde{n}^{(1)} \tilde{f}_{0}-\frac{1}{2} n_{0} \tilde{f}_{0}^{2} \int \frac{d \mathbf{k}_{1}}{(2 \pi)^{3}}\left(\frac{1}{E_{1}} \operatorname{coth} \frac{3 E_{1}}{2}-\frac{1}{\epsilon_{1}}\right) \\
& =n_{0} \tilde{f}_{0}+\frac{5}{3 \pi^{3}}\left(\frac{m}{\hbar^{2}}\right)^{3 / 2} \sqrt{n_{0} \dot{f}_{0}^{3}} n_{0} \dot{f}_{0}+2 \tilde{f}_{0} \int \frac{d \mathbf{k}_{1}}{(2 \pi)^{3}} \frac{2 \epsilon_{1}+\Delta}{2 E_{1}} f_{B}\left(E_{1}\right)
\end{aligned}
$$

It is seen that the infrared-divergent term in (T.i) cancels out in the expressions of $\mu$.

We next estimate $\mu$. The last term in (T.9) is temperature dependent. and can be written as

$$
\begin{aligned}
\mu^{T} & \equiv 2 \dot{f}_{0} \int \frac{d \mathbf{k}_{1}}{(2 \pi)^{3}} \frac{2 \epsilon_{1}+\Delta}{2 E_{1}} f_{B}\left(E_{1}\right) \\
& =2 \dot{f}_{0} \int \frac{d \mathbf{k}_{\mathbf{l}}}{(2 \pi)^{3}}\left[\frac{2 \epsilon_{1}+\Delta}{2 E_{1}} f_{B}\left(E_{1}\right)-f_{B}\left(\epsilon_{1}\right)\right]+2 n_{c r}(T) \dot{f}_{0}
\end{aligned}
$$

Here $n_{\mathrm{cr}}(T)\left[=2.612\left(m k_{B} T / 2 \pi \hbar^{2}\right)^{3 / 2}\right]$ is the critical density of an ideal Bose gas at a given $T$. as given by the integral of $f_{B}\left(\epsilon_{1}\right)$ over $\mathbf{k}_{\mathbf{l}}$. The main contribution to the integral over $k_{1}$ in the first term of $(\bar{T} .10)$ comes from the region $\epsilon_{1} \lesssim \Delta$. where $E_{1}$ differs significantly from $\epsilon_{1}$. For temperature $T$ which satisfies

$$
k_{B} T \gg \Delta=n_{0} \tilde{f}_{0}
$$


we can use the high temperature approximation of $f_{B}(\epsilon) \simeq k_{B} T / \epsilon$ to give

$$
\begin{aligned}
\mu^{T} & \simeq 2 n_{c r}(T) \dot{f}_{0}+2 \dot{f}_{0} k_{B} T \int \frac{d \mathrm{k}_{1}}{(2 \pi)^{3}}\left[\frac{2 \epsilon_{1}+\Delta}{2 E_{1}^{2}}-\frac{1}{\epsilon_{1}}\right] \\
& =2 n_{c r}(T) \dot{f}_{0}-\frac{3}{2 \pi}\left(\frac{m}{\hbar^{3}}\right)^{3 / 2} \sqrt{n_{0} \tilde{f}_{0}^{3}} k_{B} T \\
& =\frac{8 \pi \hbar^{2} a n_{c \tau}(T)}{m}-12 \pi\left(\frac{n_{0} a^{3}}{\pi}\right)^{1 / 2} k_{B} T .
\end{aligned}
$$

where in the last step. we have used $\tilde{f}_{0}=4 \pi \hbar^{2} a / m$. The result in $(\bar{i} \cdot 12)$ is in agreement with that of Popov (see equation (17.35) of Ref.[31]) if we remember that he used the units $\hbar=2 m=k_{B}=1$.

We now calculate the total chemical potential by inserting the above result for $\mu^{T}$ into $(7.9)$, and find

$$
\begin{aligned}
\mu\left(T^{*}\right)= & \frac{4 \pi \hbar^{2} n_{0} a}{m}\left[1+\frac{40}{3}\left(\frac{n_{0} a^{3}}{\pi}\right)^{1 / 2}\right] \\
& +\frac{8 \pi \hbar^{2} a n_{c r}(T)}{m}-12 \pi\left(\frac{n_{0} a^{3}}{\pi}\right)^{1 / 2} k_{B} T .
\end{aligned}
$$

The first term is the $T=0$ result already derived in (6.35). except that $n_{0}(T)$ is now temperature dependent. The new temperature-dependent parts consist of a HartreeFock term $\left[8 \pi \hbar^{2} a n_{: T}(T) / m\right]$ and another correction term which is linear in both $T$ and $\sqrt{n_{0} a^{3}}$. The second-order B-P approximation introduces a small correction (about 0.3 percent for ${ }^{87} \mathrm{Rb}$ atoms under the condition of Ref.[1]) to the chemical potential with respect to that in the first-order Popov approximation. We emphasize that ( $7.1: 3)$ has been derived assuming $n_{0} \dot{f}_{0} \ll k_{B} T$, which is valid as long as the temperature $T$ is not too low compared to $T_{c}$, namely

$$
T \gg 3.793\left(n^{1 / 3} a\right) T_{c}
$$

It can be estimated that for $a=5.3 \hat{A}$ and $n=2.6 \times 10^{12} \mathrm{~cm}^{-3}$ (taken from [1] for ${ }^{87} \mathrm{Rb}$ in the center of the trap), $n^{1 / 3} a=0.0073$ and the condition $(7.14)$ implies that $T \gg 0.027 T_{c}$. 


\subsection{Expansions of $\Sigma^{T}\left(\mathbf{k}, \omega=E_{k}+\delta\right)$ for small $\mathbf{k}$}

We are most interested in the behavior of the Green's function near the single-particle poles. However. as mentioned earlier. we cannot set $\omega=E_{k}$ and expand $\Sigma\left(\mathrm{k} . E_{k}\right)$ in the small momentum limit. due to the difficulty associated with the order of limits taken. In an attempt to overcome this problem. we introduce a gap and expand the self-energies around $\omega=E_{k}+\delta$ instead of $\omega=E_{k}$. After this expansion is done. we take the $\delta \rightarrow 0$ limit. In this approach. we are not taking the limits $\mathbf{k} \rightarrow 0$. $\dot{\sim} \rightarrow 0$ at the same time. but taking the limit $\mathrm{k} \rightarrow 0$ first. See the end of Section 7.2 for some further comments on this.

Following this procedure. we arrive at the following results (again using MAPLE):

$$
\begin{aligned}
\lim _{\varepsilon \rightarrow 0} \Sigma_{11}^{T}\left(\mathrm{k} . \pm E_{k} \pm \delta\right) & =a_{0} \pm a_{1} k+\left[a_{2} \pm a_{3}\left(\delta^{-1}\right)+a_{4}\left(\delta^{-2}\right)\right] k^{2} \\
\lim _{\delta \rightarrow 0} \Sigma_{12}^{T}\left(\mathbf{k} \cdot E_{k}+\delta\right) & =b_{0}+b_{1} k+\left[b_{2}+b_{3}\left(\delta^{-1}\right)+b_{4}\left(\delta^{-2}\right)\right] k^{2}
\end{aligned}
$$

with

$$
\begin{aligned}
a_{0}= & -\frac{n_{0} f_{0}^{2}}{2 \pi^{2}} \int_{0}^{\infty} d k_{1} k_{1}^{2} \frac{2 \epsilon_{1}^{2}+\Delta^{2}}{E_{1}^{3}} f_{B}\left(E_{1}\right)+\frac{\dot{f}_{0}}{\pi^{2}} \int_{0}^{\infty} d k_{1} k_{1}^{2} \frac{\epsilon_{1}+\Delta}{E_{1}} f_{B}\left(E_{1}\right) \\
a_{1}= & -\frac{\sqrt{2 \Delta} n_{0} f_{0}^{2}}{2 \pi^{2}} \frac{\hbar}{\sqrt{2 m}} \int_{0}^{\infty} d k_{1} k_{1}^{2}\left(-2 \epsilon_{1} \Delta^{2}+\Delta \epsilon_{1}+\epsilon_{1}^{3}\right) \frac{1}{E_{1}^{5}} f_{B}\left(E_{1}\right) \\
a_{2}= & -\frac{n_{0} f_{0}^{2}}{12} \frac{\hbar^{2}}{2 m} \int_{0}^{\infty} d k_{1} k_{1}^{2}\left(-2 \epsilon_{1}^{9}-24 \epsilon_{1}^{8} \Delta-47 \epsilon_{1}^{5} \Delta^{2}+48 \epsilon_{1}^{5} \Delta^{3}\right. \\
a_{3}= & \frac{n_{0} f_{0}^{2}}{3 \pi^{2}} \frac{\hbar^{2}}{2 m} \int_{0}^{\infty} d k_{1} k_{1}^{2}\left(2 \epsilon_{1}^{5} \Delta-\epsilon_{1}^{4} \Delta^{2}-10 \epsilon_{1}^{3} \Delta^{3}\right) \frac{1}{E_{1}^{7}} f_{B}\left(E_{1}\right) \\
a_{4}= & \frac{n_{0} f_{0}^{2}}{3 \pi^{2}} \frac{\hbar^{2}}{2 m} \int_{0}^{\infty} d k_{1} k_{1}^{2}\left(12 \epsilon_{1}^{7}+80 \epsilon_{1}^{6} \Delta+171 \epsilon_{1}^{5} \Delta^{2}\right. \\
& \left.+135 \epsilon_{1}^{4} \Delta^{3}+34 \epsilon_{1}^{3} \Delta^{4}\right) \frac{1}{E_{1}^{7}} f_{B}\left(E_{1}\right)
\end{aligned}
$$

and

$$
b_{0}=-\frac{n_{0} \tilde{f}_{0}^{2}}{2 \pi^{2}} \int_{0}^{\infty} d k_{1} k_{1}^{2} \frac{-4 \epsilon_{1} \Delta+\Delta^{2}}{E_{\mathrm{l}}^{3}} f_{B}\left(E_{1}\right)-\frac{n_{0} \dot{f}_{0}^{2}}{2 \pi^{2}} \int_{0}^{\infty} d k_{1} k_{1}^{2} \frac{1}{E_{1}} f_{B}\left(E_{1}\right) \tau
$$




$$
\begin{aligned}
b_{1}= & 0 \\
b_{2}= & -\frac{n_{0} \tilde{f}_{0}^{2}}{\left[2 \pi^{2}\right.} \frac{\hbar^{2}}{2 m} \int_{0}^{\infty} d k_{1} k_{1}^{2}\left(-8 \epsilon_{1}^{8} \Delta-11 \epsilon_{1}^{\top} \Delta^{2}+40 \epsilon_{1}^{6} \Delta^{3}+44 \epsilon_{1}^{\bar{j}} \Delta^{4}\right. \\
& \left.\quad-16 \epsilon_{1}^{-1} \Delta^{5}+32 \epsilon_{1}^{3} \Delta^{6}\right) \frac{1}{E_{1}^{11}} f_{B}\left(E_{1}\right) \\
b_{3}= & 0 \\
b_{4}= & a_{4} .
\end{aligned}
$$

We can approximate $f_{B}\left(E_{1}\right)$ for $T \sim T_{c}$ by $k_{B} T / E_{1}$ for $E_{1} \ll k_{B} T$. which limits the following results to high temperatures. In this approximation. we can evaluate the $a$ and $b$ s:

$$
\begin{aligned}
& a_{0}=2 n_{c r} \dot{f}_{0}-\frac{\sqrt{2 n_{0} \dot{f}_{0}^{3}}}{\pi^{2}}\left(\frac{m}{\hbar^{2}}\right)^{3 / 2} k_{B} T\left[\frac{3 \sqrt{2} \pi}{4}+D_{2}\right] \\
& a_{1}=-\frac{\sqrt{2}}{\pi^{2}}\left(\frac{m}{\hbar^{2}}\right) \dot{f}_{0} k_{B} T\left[\frac{\sqrt{2} \pi}{16}-D_{2}\right] \\
& a_{2}=-\frac{\sqrt{2}}{12 \pi^{2}}\left(\frac{m}{\hbar^{2}}\right)^{1 / 2} \sqrt{n_{0} \dot{f}_{0}^{3}} \frac{k_{B} T}{\Delta}\left[-\frac{t 03 \sqrt{2} \pi}{4096}+5 D_{2}+3.2 D_{4}\right] \\
& b_{0}=-\frac{\sqrt{2 n_{0} f_{0}^{3}}}{\pi^{2}}\left(\frac{m}{\hbar^{2}}\right)^{3 / 2} k_{B} T\left[0+D_{2}\right] \\
& b_{2}=-\frac{\sqrt{2}}{12 \pi^{2}}\left(\frac{m}{\hbar^{2}}\right)^{1 / 2} \sqrt{n_{0} \dot{f}_{0}^{3}} \frac{k_{B} T}{\Delta}\left[\frac{685 \sqrt{2} \pi}{4096}-D_{2}+3.2 D_{4}\right] .
\end{aligned}
$$

where $D_{2}$ and $D_{4}$ denote two infrared-divergent dimensionless integrals:

$$
\begin{aligned}
& D_{2}=\int_{0}^{\infty} d x \frac{1}{x^{2}\left(x^{2}+2\right)^{2}} \\
& D_{4}=\int_{0}^{x} d x \frac{1}{x^{4}\left(x^{2}+2\right)^{6}} .
\end{aligned}
$$

We recall that in Chapter 5 . the quantity $\Lambda_{k}^{ \pm}$near the poles $\omega= \pm E_{k}$ can be expressed in terms of $\Sigma_{11}^{ \pm}\left(\mathbf{k}, \pm E_{k}\right)$ and $\Sigma_{12}\left(\mathbf{k}, E_{k}\right)$ as

$$
\begin{aligned}
\Lambda_{k}^{ \pm}= \pm & \frac{1}{2}\left(\Sigma_{11}^{+}-\Sigma_{11}^{-}\right)+\frac{\epsilon_{k}}{2 E_{k}}\left(\Sigma_{11}^{+}+\Sigma_{12}^{-}-2 \mu^{(2)}\right) \\
& +\frac{\Delta}{2 E_{k}}\left(\Sigma_{11}^{+}+\Sigma_{11}^{-}-2 \mu^{(2)}-2 \Sigma_{12}^{(2)}\right) .
\end{aligned}
$$


The Bose-factor-independent part has been already calculated in Chapter 6. where we find [see Eqs.(6.24) and (6.29)]

$$
. ._{k}^{0 . \pm}=\frac{28}{3}\left(\frac{n_{0} a^{3}}{\pi}\right)^{1 / 2} E_{k} .
$$

The temperature-dependent part (associated with the Bose factors) of.$_{k}^{T . \pm}$ can be calculated using the corresponding temperature-dependent self-energies $\Sigma_{-11}^{T}\left(k . \pm E_{k}\right)$ and $\Sigma_{12}^{T}\left(k \cdot E_{k}\right)$ given by $(T .15)-(T .32)$ and the chemical potential given by (T.12). To first order in $k$. we obtain

$$
\begin{aligned}
I_{k}^{T . \pm}= & \frac{1}{2}\left(2 a_{1}\right) k+\frac{1}{4 \sqrt{\Delta}}\left(\frac{\hbar^{2}}{m}\right)^{1 / 2}\left(2 a_{0}-2 \mu^{T}\right) k \\
& +\frac{\sqrt{\Delta}}{2}\left(\frac{m}{\hbar^{2}}\right)^{1 / 2}\left[2 a_{2}+2 a_{4}\left(\delta^{-2}\right)-2 b_{2}-2 b_{4}\left(\delta^{-2}\right)\right] k \\
= & -\frac{31}{384 \pi}\left(\frac{m}{\hbar^{2}}\right) \dot{f}_{0}\left(k_{B} T\right) k \\
= & -\frac{31}{96}\left(k_{B} T\right) a k .
\end{aligned}
$$

We note that in $\Lambda_{k}^{T . \pm}$. the infrared divergent terms $D_{2}, D_{4} . O\left(\delta^{-1}\right)$ and $O\left(\delta^{-2}\right)$ all cancel out exactly to lowest order in $k$. just as we showed in the $T=0$ case in Chapter 6.

Combination of $(\bar{T} .36)$ and $(\bar{T} .37)$ yields the following result of $.1_{k}^{ \pm}$:

$$
\begin{aligned}
._{k}^{ \pm} & =\Lambda_{k}^{0 . \pm}+._{k}^{T . \pm} \\
& =\frac{28}{3}\left(\frac{n_{0} a^{3}}{\pi}\right)^{1 / 2} \hbar c k-\frac{31}{96}\left(k_{B} T\right) a k .
\end{aligned}
$$

This immediately leads to the following result for the energy spectrum of excitations in the second-order B-P approximation:

$$
\begin{aligned}
E_{k}^{(2)}(T) & =E_{k}^{(1)}(T)+\Lambda_{k}^{+}(T) \\
& =\hbar c^{(1)}(T) k\left[1+\frac{28}{3}\left(\frac{n_{0}(T) a^{3}}{\pi}\right)^{1 / 2}\right]-\frac{31}{96}\left(k_{B} T\right) a k
\end{aligned}
$$


Here

$$
\begin{aligned}
& \left.E_{k}^{(1)}(T)=\sqrt{\epsilon_{k}^{2}+2 \epsilon_{k} n_{0}(T) \dot{f}_{0}} \simeq \hbar c^{(1)}(T) k \quad \text { (for small } k\right) \\
& c^{(1)}(T)=\sqrt{\frac{n_{0}(T) \dot{f}_{0}}{m}}=\sqrt{\frac{4 \pi \hbar^{2} a n_{0}(T)}{m^{2}}}
\end{aligned}
$$

are the first-order excitation energy and phonon velocity. respectively. obtained in the Popov approximation of Chapter 3. Equation (7.39) shows that for small $k$. the quasiparticles are still phonons. with a velocity given by

$$
c^{(2)}(T)=c^{(1)}(T)+\frac{28}{3}\left(\frac{n_{0}\left(T^{\prime}\right) a^{3}}{\pi}\right)^{1 / 2} c^{(1)}(T)-\frac{31}{96} \frac{a}{\hbar} k_{B} T .
$$

The second term in $(\bar{\tau} .+2)$ corresponds to the $T=0$ result derived in Chapter 6 . but now is a temperature-dependent quantity through the condensate density $n_{0}(T)$. The new correction term is proportional to $k_{B} T$. but independent of $n_{0}(T)$.

The relative importance of the correction terms can be estimated for ${ }^{87} \mathrm{Rb}$ atoms $(a=53) \AA$ and $\left.n=2.6 \times 10^{12} \mathrm{~cm}^{-3}[1]\right)$ as follows. Denoting the second and third terms in $(\bar{\tau} .+2)$ as $\delta c$ and $\delta c^{\prime}$. respectively. we have

$$
\begin{aligned}
\delta c & \equiv \frac{28}{3}\left(\frac{n_{0}(T) a^{3}}{\pi}\right)^{1 / 2} \sqrt{\frac{4 \pi \hbar^{2} a n_{0}(T)}{m^{2}}} \\
& \simeq 0.0033:\left[1-\left(\frac{T}{T_{\varepsilon}}\right)^{3 / 2}\right] c^{(1 j}(0) .
\end{aligned}
$$

and

$$
\begin{aligned}
\delta c^{\prime} & \equiv-\frac{31}{96} \frac{a}{\hbar} k_{B} T \\
& \simeq 0.026 \frac{T}{T_{c}} c^{(\mathrm{l})}(0)
\end{aligned}
$$

where $c^{(1)}(0)$ is given by

$$
c^{(1)}(0)=\sqrt{\frac{4 \pi \hbar^{2} a n}{m}} \simeq 0.031(\mathrm{~cm} / \mathrm{s})
$$


As seen from $(T .43)$ and $(T .44)$. the second-order corrections are very small for a dilute ${ }^{87} \mathrm{Rb}$ atomic gas.

Since $c^{(1)}(T) \rightarrow 0$ as $T \rightarrow T_{c}$. one sees that the phonon velocity takes a small negative value near $T_{\varepsilon}$. This unphysical result is due to the fact that $(7.42)$ is derived assuming the correction terms are small, namely

$$
\left(\frac{4 \pi \hbar^{2} n_{0} a}{m^{2}}\right)^{1 / 2} \gg \frac{31}{96} \frac{a}{\hbar} k_{B} T .
$$

This condition is equivalent to

$$
T_{c}-T \gg\left(n^{1 / 3} a\right) T_{\varepsilon}
$$

The last inequality also defines the temperature range where the first-order Popor approximation is valid [see (3.59)]. Such a coincidence is not unexpected because in the second-order B-P approximation. we use the first-order Popov propagators. which are valid only if $(T .4 T)$ is true. Therefore the results in the B-P approximation are subject to the same limitation near $T_{c}$. We should also emphasize that in deriving $(7 .+2)$. we have used the high-temperature approximation. which really means that it is subject to the condition $(\boldsymbol{T} .1 .1)$. Together with ( $\vec{\imath} .4 i)$. this limits the validity of our results to temperatures which are not too close to either $T=0$ or $T=T_{:}$.

As far as we know. $(7.42)$ is a new result. Moreover, we have shown for the first time that the infrared-divergent terms cancel out in physical quantities in the long wavelength limit at finite temperature, just as in the case of zero temperature. However. it is not clear at present whether these infrared-divergent terms cancel out in physical quantities in general. i.e.. not just in the long wavelength limit. This could be checked by using our method to calculate the self-energies to higher order in $k$.

As pointed out by Stoof [47], the infrared divergence we found in the secondorder approximation might be closely related to the vanishing of $\Sigma_{12}\left(k \rightarrow 0 . \omega^{\prime}=\right.$ 0 ), a rigorous result (i.e. to all order in perturbation expansion) first shown by Nepomnyashchii et al in 1974 [48]. More precisely, one finds that $\Sigma_{12}(k, \omega=0)$ 
approaches zero as

$$
\Sigma_{12}(k, \omega=0) \rightarrow \begin{cases}\frac{1}{\ln k} & \text { at } T=0 \\ k & \text { at } T \neq 0\end{cases}
$$

Eq.(T.48) suggests that in the long wavelength limit. the self-energies. in a rigorous theory, take the following asymptotic form:

$$
\Sigma(\mathbf{k} \rightarrow 0 . \omega=0) \times \begin{cases}\frac{1}{1+\ln k} & \text { at } T=0 . \\ \frac{1}{1+\frac{1}{k}} & \text { at } T \neq 0 .\end{cases}
$$

These contain the singular infrared-divergent part in the denominator.

What we found in this thesis. however, is that. if expanded to first order. the self-energies $\Sigma(\mathbf{k} \rightarrow 0 . \omega=0)$ are finite. On the other hand. if we go to second order. the self-energies $\Sigma(\mathbf{k} \rightarrow 0 . \omega=0)$ are infinite. due to divergent terms $D_{1}$ at $T=0$ [see Eq.(6.16)] and $D_{2}$ at $T \neq 0$ [see Eq.(7.33)]. Eqs.(6.16) and (7.33) suggest that the types of divergent terms we found in the self-energies are given by

$$
\Sigma^{(2)}(k \rightarrow 0 . \omega=0) \rightarrow \begin{cases}\ln k & \text { at } T=0 \\ \frac{1}{k} & \text { at } T \neq 0\end{cases}
$$

A comparison of $(T .48)$ and $(T .49)$ suggests the origin of the divergent terms found in our second-order calculations. In the perturbative calculation we have developed. one may be effectively expanding Eq. $(\bar{T} .49)$ in powers of $\ln k$ or $1 / k$, arriving at results consistent with $(7.50)$.

$$
\Sigma(k \rightarrow 0, \omega=0) \propto \begin{cases}1+O(\ln k)+O\left(\ln ^{2} k\right)+\cdots & \text { at } T=0 . \\ 1+O\left(\frac{1}{k}\right)+O\left(\frac{1}{k^{2}}\right)+\cdots & \text { at } T \neq 0 .\end{cases}
$$

Here the first term corresponds to the first-order self-energy result and the second term corresponds to the second-order self-energy result, and so on. This scenario gives an explanation of why our second-order self-energies contain the types of divergence shown in Eq.(?.50). 
The above analysis puts a question mark on the traditional perturbation approach we have used. However, the fact that these divergent terms all cancel out in the end seems to suggest that the singular parts of the self-energies do not contribute to physical quantities. at least to second-order in $\dot{f}$. and therefore. the results we obtained in Chapter 6 and in the present chapter still remain meaningful. The exact cancellation also implies that the problem associated with infrared divergence may be solved by some kind of re-formulation. Recently. Bijlsma and Stoof [4.5] have studied the problem using the renormalization group ( $R G$ ) techniques, and shown how. in principle, the infrared divergence problems can be solved using such techniques.

As we remarked at the beginning of this section, we changed the order of limits $k \rightarrow 0$ and $\omega \rightarrow 0$ by introducing a gap $\delta$ with respect to the Popor quasiparticle energy $E_{k}$ in the frequency $\omega$. As one can easily see from $(\overline{7} .15)$ and $(7.16)$. had we taken the limit $\omega \rightarrow 0$ (or $\delta \rightarrow 0$ ) together with $k \rightarrow 0$. we would get singular results. due to the presence of $k^{2} / \delta$ and $k^{2} / \delta^{2}$ terms. The change of the order of limits might have changed the physics contained in $a_{0}$ and $b_{0}$ (such as the type of contributions

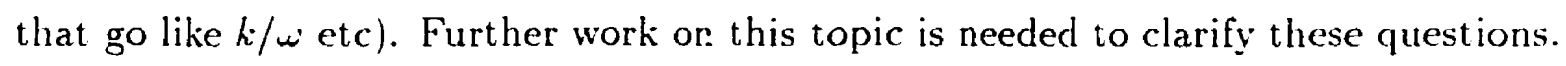

\subsection{Damping of phonons at high temperature}

As first shown by Beliaer [34] and rederived in Chapter 6. at zero temperature. the damping of low-momentum phonons is proportional to $k^{5}$ [see Eq.(6.31)]. We can use the finite-temperature results of the present chapter to calculate the imaginary part of the self-energies. From these, we can calculate the phonon damping at high temperatures, close to $T_{c}$. We briefly sketch these calculations. the details of which will be reported elsewhere [49].

We first calculate the imaginary parts of $\Sigma_{11}$ and $\Sigma_{12}$ at the pole $\omega=E_{k}$. starting from the results given in ( 7.1$)$ and (7.2). The imaginary part of $\Sigma_{11}^{T}\left(\mathbf{k} . E_{k}+i \eta\right)$ is given by

$$
\operatorname{Im} \Sigma_{11}^{+} \equiv \operatorname{Im} \Sigma_{11}\left(\mathbf{k}, E_{k}+i \eta\right)
$$




$$
\begin{aligned}
=n_{0} \dot{f}_{0}^{2} \int & \frac{d \mathbf{q}}{(2 \pi)^{3}} \frac{f_{B}\left(E_{1}\right)}{E_{1} E_{2}}(-\pi)\left[g\left(E_{1}, E_{2}\right) \delta\left(E_{k}-E_{1}-E_{2}\right)\right. \\
& -g\left(-E_{1} \cdot-E_{2}\right) \delta\left(E_{k}+E_{1}+E_{2}\right)+g\left(-E_{1} . E_{2}\right) \delta\left(E_{k}+E_{1}-E_{2}\right) \\
& \left.-g\left(E_{1},-E_{2}\right) \delta\left(E_{k}-E_{1}+E_{2}\right)\right] \\
=-\frac{n_{0} \tilde{f}_{0}^{2}}{4 \pi}\left(\frac{m}{\hbar^{2}}\right)^{2} \frac{1}{k} & {\left[\left.\int_{0}^{E_{k}} d E_{1} \frac{g\left(E_{1}, E_{2}\right)}{\left(\epsilon_{1}+\Delta\right)\left(\epsilon_{2}+\Delta\right)} f_{B}\left(E_{1}\right)\right|_{E_{2}=E_{k}-E_{1}}\right.} \\
& +\left.\int_{0}^{\infty} d E_{1} \frac{g\left(E_{1},-E_{2}\right)}{\left(\epsilon_{1}+\Delta\right)\left(\epsilon_{2}+\Delta\right)} f_{B}\left(E_{1}\right)\right|_{E_{2}=E_{k}+E_{1}} \\
& -\left.\int_{E_{k}}^{x} d E_{1} \frac{g\left(E_{1},-E_{2}\right)}{\left(\epsilon_{1}+\Delta\right)\left(\epsilon_{2}+\Delta\right)} f_{B}\left(E_{1}\right)\right|_{E_{2}=E_{1}-E_{k}}
\end{aligned}
$$

The function $g\left(E_{1}, E_{2}\right)$ is defined in $(5.30)$ as well as at the beginning of the present chapter following (T.2). Im $\Sigma_{11}^{+}$in (T.5.3) consists of three parts, which involve different lower and upper limits to ensure the positiveness of $E_{2}$. In arriving at (7.5\%3). we have made use of the following property of the $\delta$-function:

$$
\int d x f_{1}(x) \delta\left[f_{2}(x)\right]=\left[f_{1}(x)\left|\frac{d f_{2}(x)}{d x}\right|^{-1}\right]_{x=x_{0}}
$$

where $x_{0}$ is the solution of $f_{2}(x)=0$. In the high temperature limit. we approximatc $f_{B}\left(E_{1}\right)$ by $k_{B} T / E_{1}$, and after some manipulations (for details, see [-19]) we finally. arrive at the following Taylor expansion of $\operatorname{Im} \Sigma_{11}^{+}\left(\mathbf{k} . E_{k}\right)$ for small $k$ :

$$
\begin{aligned}
\operatorname{Im} \Sigma_{11}^{+}= & -\frac{n_{0} \tilde{f}_{0}^{2}}{4 \pi}\left(\frac{m}{\hbar^{2}}\right)^{2} \frac{k_{B} T}{k}\left[2 \bar{D}_{k}+\left(4-\frac{\pi}{2}-2 \bar{D}_{k}\right) \frac{E_{k}}{د}+(\pi-2) \frac{E_{k}^{2}}{\Delta^{2}}\right. \\
& \left.+\left(\frac{75 \pi-400}{96}+\bar{D}_{k}\right) \frac{E_{k}^{3}}{\Delta^{3}}+O\left(E_{k}^{4}\right)\right] .
\end{aligned}
$$

Here $\bar{D}_{k}$ is a dimensionless divergent integral defined by

$$
\bar{D}_{k} \equiv \frac{1}{k_{B} T} \int_{0}^{E_{k}} d E_{1} \frac{\Delta^{2}}{E_{1}^{2}+\Delta^{2}} f_{B}\left(E_{1}\right)
$$

Following a similar procedure, we can also work out the Taylor expansion of 
$\operatorname{Im} \Sigma_{11}\left(-\mathbf{k} .-E_{k}\right)$ and $\operatorname{Im} \Sigma_{12}\left(\mathrm{k}, E_{k}\right)$ for small $k$, with the following results:

$$
\begin{aligned}
\operatorname{Im} \Sigma_{-11}^{-} \equiv & {\left[m \Sigma_{11}\left(-\mathbf{k} .-E_{k}-i \eta\right)\right.} \\
= & -\frac{n_{0} \tilde{f}_{0}^{2}}{4 \pi}\left(\frac{m}{\hbar^{2}}\right)^{2} \frac{k_{B} T}{k}\left[2 \bar{D}_{k}+\left(4-\frac{\pi}{2}+2 \bar{D}_{k}\right) \frac{E_{k}}{د}-(\pi-2) \frac{E_{k}^{2}}{\Delta^{2}}\right. \\
& \left.+\left(\frac{7 j \pi-112}{96}-\bar{D}_{k}\right) \frac{E_{k}^{3}}{\Delta^{3}}+O\left(E_{k}^{4}\right)\right] \\
\operatorname{Im} \Sigma_{12} \equiv & \operatorname{Im} \Sigma_{12}\left( \pm \mathbf{k} . \pm E_{k} \pm i \eta\right) \\
= & -\frac{n_{0} \tilde{f}_{0}^{2}}{4 \pi}\left(\frac{m}{\hbar^{2}}\right)^{2} \frac{k_{B} T}{k}\left[2 \bar{D}_{k}+\left(t-\frac{\pi}{2}\right) \frac{E_{k}}{د}-\bar{D}_{k} \frac{E_{k}^{2}}{\Delta^{2}}\right. \\
& \left.+\frac{111 \pi-2.56}{96} \frac{E_{k}^{3}}{\Delta^{3}}+O\left(E_{k}^{4}\right)\right] .
\end{aligned}
$$

Using ( $\overline{7} .54) .(7.56)$ and $(\bar{T} .57)$ in $(\bar{T} .37)$, we find that the imaginary part of the quantity $I_{k}^{+}$as well as the imaginary part of the excitation energy $E_{k}$ in the lowmomentum limit is given by the very simple expression

$$
\operatorname{Im} E_{k}^{B P}=\operatorname{Im} \Lambda_{k}^{+}=-\frac{3 \pi a}{8} k_{B} T k
$$

We remark that, unlike the $T=0$ case discussed in Chapter 6 . the imaginary parts of the self-energies exhibit divergent terms at finite temperature. This can be seen from the quantity $\bar{D}_{k}$ which appears in various terms in the expressions for $\operatorname{Im} \Sigma_{11}^{ \pm}$ and $\operatorname{Im} \Sigma_{12}$ given above. However. these divergent terms all cancel out exactly in the final expression $(\bar{T} .58)$ for the damping. This shows. once again. that the singular parts of the self-energies do not contribute to long-wavelength physical quantities in the Beliaev-Popov approximation at finite temperatures.

The result in ( 7.58$)$ shows that the damping of phonons is proportional to $T k$ in the high temperature region near $T_{c}$. We believe this result is new. We call attention to the fact that temperature-dependent correction to the real part of the phonon energy given by the last term in ( 7.39$)$ is very similar to the damping given by $(T .58)$. The phonon width in the low temperature limit has been calculated by Popov [31] using a hydrodynamical Hamiltonian approach. In this limit, the phonon width is proportional to $T^{4} k$. 


\section{Chapter 8}

\section{Concluding remarks}

Wost of this thesis has concentrated on the technical details of evaluating the diagonal and off-diagonal Beliaev self-energies to second order in the interaction. In this bricf concluding chapter, we would like to put some of our results into a larger context by discussing the connections to recent field-theoretic literature on uniform interacting Bose-condensed gases.

The first-order Popov approximation worked out in detail in Chapter 3 is a simplified version of the complete self-consistent Hartree-Fock-Bogoliubov (HFB) approximation for the single-particle self-energies. As we mentioned earlier. the latter is known to give rise to an energy gap in the long wavelength excitation spectrum. However, the HFB approximation is of special interest since it can be used to generate a density-response function (by functional differentiation with respect to an auxiliary field) which exhibits the same spectrum as the single-particle Green's function computed in the Beliaev-Popov approximation. This is proven in detail by Cheung and Griffin [29] at finite temperatures and has been recently reviewed by Griffin [41] in the context of trapped atomic gases. We should also note that the Popov approximation to the self-consistent HFB has been recently formulated for a Bose gas trapped in an external potential well [50], extending the work in Chapter 3.

In Chapters 6 and $i$, the second-order self-energies exhibited infrared-divergent terms both at $T=0$ (Beliaev) and $T \neq 0$ (Popov). We showed explicitly that these divergent contributions cancel out in thermodynamic properties like the chem- 
ical potential. as well as in the low frequency excitation energy and damping. In this thesis. we have not discussed the physics of such divergent terms (see Section 6.3 of Refs. [8] and [30]) or alternative formulations in which they do not explicitly appear (see however the remarks at the end of Section 7.2). There is an extensive literature on this topic. going back to the pioneer work of Gavoret and Nozières [2.2] at $T=0$. We call attention to the renormalization group type of calculation given by Popov [31] which derives a renormalized "quantum hydrodynamic" description of long wavelength modes which is free of such divergent terms. Related recent studies using formal renormalization group (RG) techniques at $T=0[51]$ and near $T_{c}[4.5]$ have addressed this problem in a systematic way.

As we noted in the introduction of this thesis. we have concentrated on calculating the single-particle Green's functions at finite temperatures. However. it is known that in the presetice of a Bose broken symmetry. the single-particle and density fluctuations are hybridized, leading to the same poles for both single-particle Green's functions and the density response function. The so-called "dielectric formalism" developed by Wa and Woo [52] (for a review. see Chapter 5 of Ref.[8]) is a diagrammatic approach which enables one to develop approximations for "regular" functions (the "regular" self-energy diagrams. by definition. can not be split into two by cutting a single interaction line) which will lead to $G_{\alpha, j}(\mathbf{k} . \omega)$ and ${ }_{{ }_{n n} n}(\mathbf{k} . \omega)$ having the same spectrum (but with different weights) in a Bose-condensed system. This dielectric formalism has been used in the so-called one-loop approximation to discuss the excitations of a dilute Bose gas at $T=0$ by Wong and Gould [53] and at $T \neq 0$ by Talbot and Giriffin [54]. At $T=0$, this one-loop approximation has been shown to give the second-order Beliaev spectrum (see Chapter 6 of this thesis).

We hope that the explicit calctlations given in this thesis (and especially Chapters 6 and 7 ) will be useful in the on-going effort to provide a more complete and satisfactory understanding of excitations in a Bose-condensed gas.

In principle, the thermal Green's functions can be used to calculate the thermodynamic quantities, such as the pressure, the specific heat etc. This was done for the first-order Popov approximation (in connection with Chapter 3) by Popov [32]. We 
have not carried out these calculations for the second-order Beliaev-Popov approximation at finite temperatures (in connection with Chapter $i$ ) in this thesis. One of the reasons is that to do this. one needs the Green's functions for all momenta and frequencies. which is difficult to obtain in analytical form. In this thesis. we have given analytical expressions for the Green`s functions near the poles $\omega= \pm E_{k}$. which allowed us to determine the explicit excitation energy spectrum. Nevertheless. it would be interesting to calculate some thermodynamic quantities by numerical methods, using the formalism developed in this thesis. 


\section{Appendix A}

\section{Scattering theory and the t-matrix for Bose systems}

The typical inter-atomic potential involves a hard core. This poses a problem for the perturbation theory in terms of the bare potential $v$. Since $v$ can be large. the first few terms in such perturbation expansion are no longer sufficient. Indeed. one has to sum over an infinite number of terms. This Appendix reviews the so-called ladder approximation for the many-body scattering amplitude $\Gamma$ in a Bose gas. In this approximation. $\Gamma$ involves the sum over all the ladder diagrams to infinite order in $r$. taking into account the repeated scattering of two particles in a gas. To understand this multiple scattering of two atoms in the presence of many other atoms. one has to first understand the simpler problem of multiple scattering of two atoms in a racuum. since it can be shown that the many-body scattering amplitude $\Gamma$ is related to the free space or vacuum scattering amplitude (see Chapter 4 of Ref.[1:3]).

\section{A.1 Multiple scattering in vacuum}

We consider two particles of mass $m$ interacting in vacuum via potential $v(\mathbf{x})$. The Schrödinger equation in the center-of-mass coordinate system is given by

$$
\frac{\hbar^{2}}{2 m^{\prime}}\left(\nabla^{2}+k^{2}\right) \phi(\mathbf{x})=v(\mathbf{x}) \phi(\mathbf{x})
$$


Here $m^{\prime}=m / 2$ is the reduced mass and $o(\mathbf{x})$ describes the wavefunction of the $t$ wo particles with a separation $\mathbf{x}$ between them. In scattering problems. it is generally convenient to rewrite equation (A.1) as an integral equation representing an incident plane wave with wavevector $\mathrm{k}$ plus an outgoing scattered wave:

$$
\circlearrowleft_{\mathbf{k}}(\mathbf{x})=e^{i \mathbf{k} \cdot \mathbf{x}}-\int d \mathbf{y} \frac{m}{4 \pi \hbar^{2}} \frac{e^{i k|\mathbf{x}-\mathbf{y}|}}{|\mathbf{x}-\mathbf{y}|} \frac{m}{\hbar^{2}} v(\mathbf{y}) \circlearrowleft_{\mathbf{k}}(\mathbf{y}) .
$$

At large $x$. i.e. far away from the influence of the potential. the scattered wavefunction take the following asymptotic form:

$$
\epsilon^{i \mathbf{k}^{\prime} \cdot \mathbf{x}}+f\left(\mathbf{k} \cdot \mathbf{k}^{\prime}\right) \frac{e^{i k^{\prime} x}}{x} \quad x \rightarrow \infty .
$$

This defines the scattering amplitude for a transition from an incident wave vector $k$ to a final wave vector $\mathbf{k}^{\prime}$. namely

$$
\begin{aligned}
f\left(\mathbf{k} \cdot \mathbf{k}^{\prime}\right) & \equiv-\frac{m}{4 \pi \hbar^{2}} \int d \mathbf{y} e^{-i \mathbf{k}^{\prime} \cdot \mathbf{y}} v(\mathbf{y}) \omega_{\mathbf{k}}(\mathbf{y}) \\
& =-\frac{m}{4 \pi \hbar^{2}} \int \frac{d \mathbf{q}}{(2 \pi)^{3}} v(\mathbf{q}) \omega_{\mathbf{k}}\left(\mathbf{k}^{\prime}-\mathbf{q}\right)
\end{aligned}
$$

where

$$
\begin{aligned}
\omega_{\mathbf{k}}\left(\mathbf{k}^{\prime}-\mathbf{q}\right) & =\int d \mathbf{y} \epsilon^{-i\left(\mathbf{k}^{\prime}-\mathbf{q}\right) \cdot \mathbf{y}} \omega_{\mathbf{k}}(\mathbf{y}) \\
v(\mathbf{q}) & =\int d \mathbf{y} \epsilon^{-i \mathbf{q} \cdot \mathbf{y}} v \cdot(\mathbf{y}) .
\end{aligned}
$$

The Schrödinger equation (A.2) may be rewritten in momentum space

$$
\begin{aligned}
\Phi_{\mathbf{k}}\left(\mathbf{k}^{\prime}\right) & =(2 \pi)^{3} \delta\left(\mathbf{k}^{\prime}-\mathbf{k}\right)-\frac{1}{2 \epsilon_{k^{\prime}}-2 \epsilon_{k}-i \eta} \int \frac{d \mathbf{q}}{(2 \pi)^{3}} v(\mathbf{q}) o_{\mathbf{k}}\left(\mathbf{k}^{\prime}-\mathbf{q}\right) \\
& =(2 \pi)^{3} \delta\left(\mathbf{k}^{\prime}-\mathbf{k}\right)-\frac{f\left(\mathbf{k}^{\prime}, \mathbf{k}\right)}{2 \epsilon_{k^{\prime}}-2 \epsilon_{k}-i \eta}
\end{aligned}
$$

where we have defined the quantity $\tilde{f}\left(\mathbf{k}, \mathbf{k}^{\prime}\right)$

$$
\tilde{f}\left(\mathbf{k}, \mathbf{k}^{\prime}\right) \equiv-\frac{4 \pi \hbar^{2}}{m} f\left(\mathbf{k}, \mathbf{k}^{\prime}\right)
$$




$$
=\int d \mathbf{q} v(\mathbf{q}) \omega_{\mathbf{k}}\left(\mathbf{k}^{\prime}-\mathbf{q}\right)
$$

We note that this definition of $\tilde{f}$ differs from the usual definition of the free-space scattering amplitude $f$ by a factor of $-4 \pi \hbar^{2} / m$. Combining (A.5) and (A.i). one obtains

$$
\tilde{f}\left(\mathbf{k}, \mathbf{k}^{\prime}\right)=v\left(\mathbf{k}^{\prime}-\mathbf{k}\right)+\int \frac{d \mathbf{q}}{(2 \pi)^{3}} \frac{v\left(\mathbf{k}^{\prime}-\mathbf{q}\right)}{2 \epsilon_{k}-2 \epsilon_{q}+i \eta} \tilde{f}(\mathbf{k} . \mathbf{q}) .
$$

which is an integral equation for $\dot{f}$ in terms of the bare potential $v$. If the potential has no bound states. as will be assumed throughout this section. then the exact scattering solutions with a given boundary condition form a complete set of states and satisfy. the following completeness relation in momentum space [5.5]:

$$
\int \frac{d \mathbf{k}}{(2 \pi)^{3}} \phi_{\mathbf{k}}\left(\mathbf{k}_{1}\right) o_{\mathbf{k}}^{*}\left(\mathbf{k}_{2}\right)=(2 \pi)^{3} \delta\left(\mathbf{k}_{1}-\mathbf{k}_{2}\right)
$$

The significance of (A.S) is that $\bar{f}\left(\mathbf{k} . \mathbf{k}^{\prime}\right)$ is well-defined even if $v(\mathbf{r})$ has a hä $\mathbf{u}$ core. The solution of (A.S) is discussed in textbooks on scattering theory. In particular in the long wavelength limit $\left(|\mathbf{k}|=\left|\mathbf{k}^{\prime}\right| \rightarrow 0\right)$. the solution of (A.S) is given by

$$
\tilde{f}\left(\mathbf{k}, \mathbf{k}^{\prime}\right)=\frac{4 \pi \hbar^{2} a}{m}+O\left(k a^{2}\right) .
$$

where $a$ is the s-wave scattering length.

\section{A.2 Ladder diagrams}

The previous discussion has been restricted to the scattering of two atoms in vacuum. We now turn to the much more complicated problem of two interacting atoms in a gas. As discussed in $[34,56,13]$, in a dilute gas, the ladder diagrams are all of equal importance. For example, there exists a set of ladder diagrams, shown in Fig.A.1 . all of which are the same order in the gas density as the lowest-order Hartree-Fock diagrams are. Therefore, in a dilute gas, we need to sum over all the ladder diagrams, yielding the so-called many-body scattering amplitude $\Gamma$, as illustrated by the hatched 


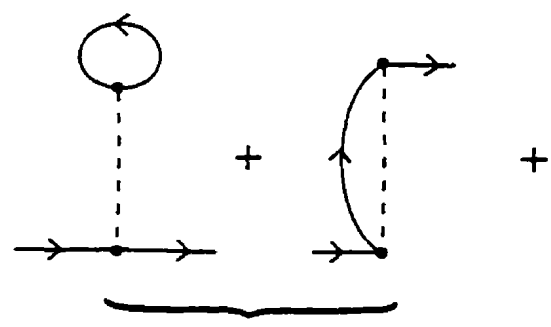

Lowest order

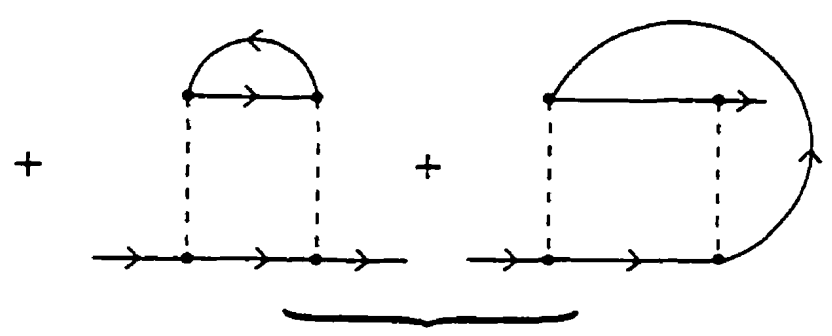

Second order

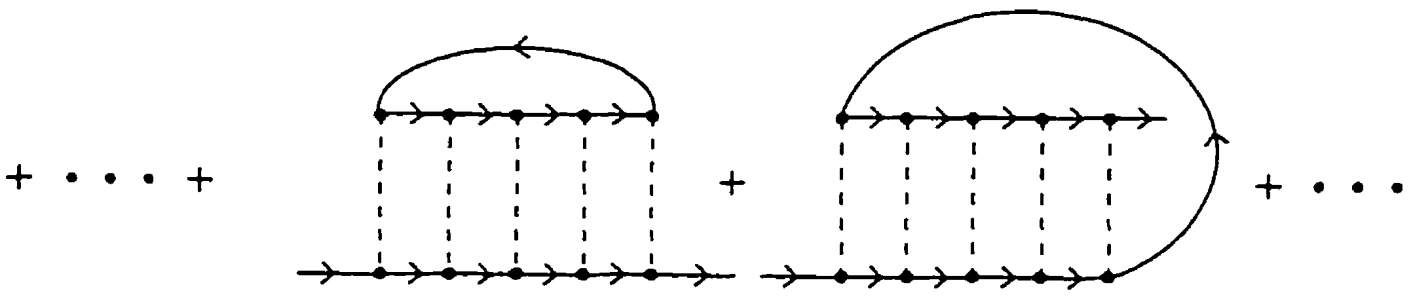

Figure A.1: Ladder diagrams that are equally important in the Hartree-Fock approximation

square in Fig.A.2. The diagrammatic definition of $\Gamma$ in Fig.A.2 which can be written explicitly as

$$
\begin{aligned}
\Gamma\left(p_{1}, p_{2} ; p_{3}, p_{4}\right) \equiv v\left(\mathbf{k}_{\mathbf{1}}-\mathbf{k}_{3}\right)-\frac{1}{3 V} \sum_{\mathbf{q}, i \omega_{7}} v(\mathbf{q}) G^{(0)}\left(\mathbf{k}_{1}-\mathbf{q}, i \omega_{1}-i \omega_{7}\right) \\
\times G^{(0)}\left(\mathbf{k}_{2}+\mathbf{q} ; i \omega_{2}+i \omega_{q}\right) \Gamma\left(p_{1}-q \cdot p_{2}+q ; p_{3}, p_{4}\right) .( \\
=v\left(\mathbf{k}_{1}-\mathbf{k}_{3}\right)+\int \frac{d \mathbf{q}}{(2 \pi)^{3}} v(\mathbf{q}) \Gamma\left(p_{1}-q, p_{2}+q: p_{3}, p_{4}\right) \\
\times \frac{1+f_{B}\left(\epsilon_{\mathbf{k}_{1}-\mathbf{q}}\right)+f_{B}\left(\epsilon_{\mathbf{k}_{2}+\mathbf{q}}\right)}{i \omega_{1}+i \omega_{2}-\left(\epsilon_{\mathbf{k}_{1}-\mathbf{q}}-\mu\right)-\left(\epsilon_{\mathbf{k}_{2}+\mathbf{q}}-\mu\right)} .
\end{aligned}
$$

The four-dimensional vector $p_{j} \equiv\left(k_{j}, i \omega_{j}\right)$ represents the momentum $k_{\jmath}$ and Matsubara frequency $i \omega_{j}$ of a particle before $(j=3,4)$ or after $(j=1,2)$ scattering, similarly, $q \equiv\left(\mathbf{q}, i \omega_{q}\right)$. Since the interatomic potential $v(\mathbf{r})$ is not time-dependent, $v(\mathbf{q})$ does not depend on the fourth component $i \omega_{q}$ of $q$, and neither does $\Gamma\left(p_{1}-q, p_{2}+q ; p_{3}, p_{4}\right)$. 


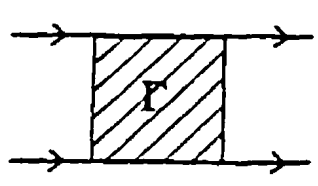

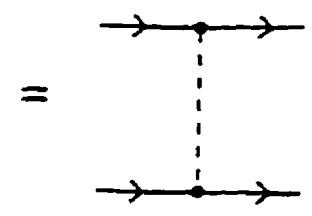

Lowest order

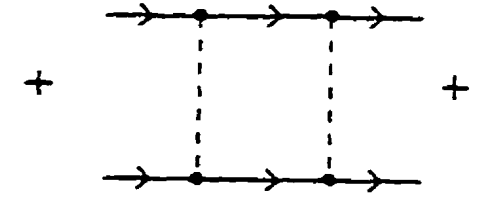

Second order
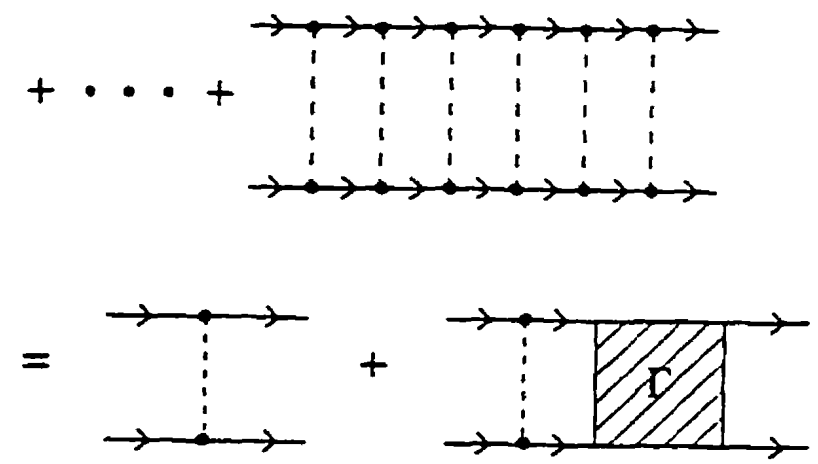

Figure A.2: Diagrammatic definition of the $t$-matrix

One can thus perform the frequency sum

$$
\sum_{i \omega_{q}} G^{(0)}\left(\mathbf{k}_{1}-\mathbf{q}, i \omega_{1}-i \omega_{\eta}\right) G^{(0)}\left(\mathbf{k}_{2}+\mathbf{q}, i \omega_{2}+i \omega_{\eta}\right)
$$

in (A.11), yielding the last factor in (A.12). Eq.(A.11) is known as the Bethe-Salpeter equation (more precisely, the ladder approximation to the Bethe-Salpeter equation). If (A.12) is iterated in powers of $v$ (perturbation theory), we obtain the sum of all ladder diagrams.

It is often more convenient to write $\Gamma$ in the center-of-momentum frame of the scattering pair of atoms. Defining the total momentum and total Matsubara frequency of the scattering pair as

$$
\begin{aligned}
& K \equiv k_{1}+k_{2}=k_{3}+k_{4}, \\
& i \omega_{N} \equiv i \omega_{1}+i \omega_{2}=i \omega_{3}+i \omega_{4},
\end{aligned}
$$


the denominator in the integral of (A.12) can rewritten as

$$
z-2\left(\epsilon_{\mathrm{k}-\mathrm{q}}-\mu\right)
$$

where the complex variable $z$ is defined by

$$
z \equiv \omega_{\omega N}-\xi_{K} \equiv i_{\omega N}-\frac{\hbar^{2} K^{-2}}{4 m} .
$$

Writing $\Gamma$ in terms of variables in the center-of-mass frame, we have

$$
\begin{aligned}
& \Gamma\left(\mathbf{k} \cdot \mathbf{k}^{\prime} \cdot \mathbf{K}: z\right) \equiv \Gamma\left(p_{1} \cdot p_{2}: p_{3} \cdot p_{4}\right) \\
& \quad=v\left(\mathbf{k}-\mathbf{k}^{\prime}\right)+\int \frac{d \mathbf{q}}{(2 \pi)^{3}} v(\mathbf{q}) \frac{F_{+}(\mathbf{K} \cdot \mathbf{k}-\mathbf{q})}{z-2\left(\epsilon_{\mathbf{k}-\mathbf{q}}-\mu\right)} \Gamma\left(\mathbf{k}-\mathbf{q} \cdot \mathbf{k}^{\prime} \cdot \mathbf{K}: z\right) .
\end{aligned}
$$

where

$$
\begin{aligned}
\mathbf{k} & \equiv \frac{\mathbf{k}_{1}-\mathbf{k}_{2}}{2} . \\
\mathbf{k}^{\prime} & \equiv \frac{\mathbf{k}_{3}-\mathbf{k}_{4}}{2} . \\
F_{+}(\mathbf{K} \cdot \mathbf{k}-\mathbf{q}) & \equiv 1+f_{B}\left(\epsilon_{\frac{1}{2} \mathrm{~K}+\mathbf{k}-\mathbf{q}}\right)+f_{B}\left(\epsilon_{\frac{1}{2} \mathbf{K}-\mathbf{k}+\mathbf{q}}\right) .
\end{aligned}
$$

Here $\mathrm{k}^{\prime}$ and $\mathrm{k}$ are the relative momenta of the scattering pair before and after the scattering. respectively: and the function $F_{+}(\mathbf{k} . \mathbf{q}, \mathbf{K})$ incorporates the effect of the Bose statistics obeyed by the atoms involved in the intermediate scattering states. Eq.(A.18) can be compared with the integral equation for the free-space scattering amplitude $\tilde{f}$ given by (A.8). The free-space scattering amplitude $\dot{f}$ describes the effect of the potential on the wavefunction of two atoms in a vacuum. while the many-body $T$-matrix $\Gamma$ describes the similar effect in a medium. that is. in the presence of other atoms in a Bose gas.

The diagrams in Fig.A.1 are redrawn in Fig.A.3 in terms of the new quantity $\Gamma$ defined in Fig.A.2. These diagrams in Fig.A.3 can be obtained by simply "replacing" $v$ (the dashed lines) in the Hartree-Fock diagrams ( $c$ and $d$ in Fig.3.1) with $\Gamma$ (the hatched squares). 


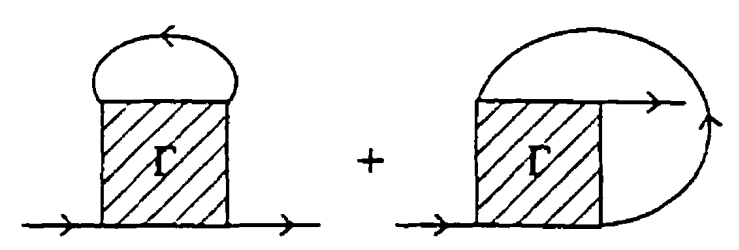

Figure A.3: Hartree-Fock self-energy diagrams in the $t$-matrix approximation

\section{A.3 Connections between $\tilde{f}$ and $\Gamma$}

We next show the connection between the free-space scattering amplitude $\bar{f}$ defined in (A. $T$ ) and the many-body scat tering amplitude $\Gamma$. The following analysis at $T \neq 0$ is a generalization of the $T=0$ discussion given by Beliaev (for Bose systems) and Fetter and Walecka (for Fermi systems) [34, 13]. Introducing the quantity $x$ defined by

$$
\Gamma\left(\mathbf{k} \cdot \mathbf{k}^{\prime} \cdot \mathbf{K}: z\right) \equiv \int \frac{d \mathbf{q}}{(2 \pi)^{3}} v(\mathbf{q}) \chi\left(\mathbf{k}-\mathbf{q} \cdot \mathbf{k}^{\prime} \cdot \mathbf{K}: z\right) .
$$

one can verify that $\chi$ satisfies the following integral equation

$$
\begin{aligned}
\chi\left(\mathbf{k}, \mathbf{k}^{\prime} \cdot \mathbf{K} ; z\right)= & (2 \pi)^{3} \delta\left(\mathbf{k}-\mathbf{k}^{\prime}\right)+\frac{F_{+}(\mathbf{K} \cdot \mathbf{k})}{z-2 \epsilon_{k}+2 \mu} \\
& \times \int \frac{d \mathbf{q}}{(2 \pi)^{3}} v(\mathbf{q}) \chi\left(\mathbf{k}-\mathbf{q}, \mathbf{k}^{\prime} \cdot \mathbf{K}: z\right) .
\end{aligned}
$$

The equation for $\chi$ may be compared with the similar equation (A.6) for $\sigma_{k}\left(k^{\prime}\right)$. which suggests that $\chi$ can be interpreted as an effective wavefunction of two particles in the Bose gas medium.

The connection of $x$ to $\dot{f}$ can be made by first considering the analogous function $\chi^{0}\left(\mathbf{k}, \mathbf{k}^{\prime}, \mathbf{K} ; z\right)$, which denotes the solution of $(\mathrm{A} .23)$ with $F_{+}(\mathbf{K}, \mathbf{k})$ replaced by unity:

$$
\begin{aligned}
\chi^{0}\left(\mathbf{k}, \mathbf{k}^{\prime}, \mathbf{K} ; z\right) \equiv & (2 \pi)^{3} \delta\left(\mathbf{k}-\mathbf{k}^{\prime}\right)+\frac{1}{z-2 \epsilon_{k}+2 \mu} \\
& \times \int \frac{d \mathbf{q}}{(2 \pi)^{3}} v(\mathbf{q}) \chi^{0}\left(\mathbf{k}-\mathbf{q}, \mathbf{k}^{\prime} . \mathbf{K} ; z\right) .
\end{aligned}
$$


The corresponding quantity $\Gamma^{0}$ is defined through $\chi^{0}$ by [see (A.22)]

$$
\Gamma^{0}\left(\mathbf{k} \cdot \mathbf{k}^{\prime} \cdot \mathbf{K}: z\right) \equiv \int \frac{d \mathbf{q}}{(2 \pi)^{3}} v(\mathbf{q}) \mathbf{\chi}^{0}\left(\mathbf{k}-\mathbf{q}, \mathbf{k}^{\prime} \cdot \mathbf{K}: z\right)
$$

We now rewrite $(A .2 t)$ for $l^{0}$ and $(A .6)$ for $\phi_{\mathbf{k}_{1}}(\mathbf{k})$ as

$$
\begin{gathered}
\left(z-2 \epsilon_{k}+2 \mu\right) \mathbf{X}^{0}\left(\mathbf{k}, \mathbf{k}^{\prime} \cdot \mathbf{K} ; z\right)-\int \frac{d \mathbf{q}}{(2 \pi)^{3}} v(\mathbf{q}) \lambda^{0}\left(\mathbf{k}-\mathbf{q} \cdot \mathbf{k}^{\prime} . \mathbf{K}: z\right) \\
=(2 \pi)^{3}\left(z-2 \epsilon_{k}+2 \mu\right) \delta\left(\mathbf{k}-\mathbf{k}^{\prime}\right) . \\
\left(2 \epsilon_{k_{1}}-2 \epsilon_{k}+i \eta\right) \odot_{\mathbf{k}_{1}}(\mathbf{k})-\int \frac{d \mathbf{q}}{(2 \pi)^{3}} v(\mathbf{q}) \odot_{\mathbf{k}_{1}}(\mathbf{k}-\mathbf{q})=0 .
\end{gathered}
$$

Multiplying $(A .26)$ by $o_{\mathrm{k}_{1}}^{*}(\mathrm{k})$ and integrating over $\mathrm{k}$. we find that the second term on the left hand side equals

$$
\begin{aligned}
\int \frac{d \mathbf{k}}{(2 \pi)^{3}} \int \frac{d \mathbf{q}}{(2 \pi)^{3}} v(\mathbf{q}) \lambda^{0}\left(\mathbf{k}-\mathbf{q} \cdot \mathbf{k}^{\prime} \cdot \mathbf{K}: z\right) \omega_{\mathbf{k}_{1}}^{*}(\mathbf{k}) \\
=\int \frac{d \mathbf{k}}{(2 \pi)^{3}} x^{0}\left(\mathbf{k} \cdot \mathbf{k}^{\prime} \cdot \mathbf{K}: z\right) \int \frac{d \mathbf{q}}{(2 \pi)^{3}} c^{\prime}(\mathbf{q}) \omega_{\mathbf{k}_{1}}^{*}(\mathbf{k}-\mathbf{q}) \\
=\int \frac{d \mathbf{k}}{(2 \pi)^{3}} l^{0}\left(\mathbf{k} \cdot \mathbf{k}^{\prime} \cdot \mathbf{K}: z\right)\left(2 \epsilon_{k_{1}}-2 \epsilon_{k}-i \eta\right) \omega_{\mathbf{k}_{1}}^{-}(\mathbf{k})
\end{aligned}
$$

In the second line, we have used the variable change $(k-q) \rightarrow k$. and in the third line, we made use of the complex conjugate of (A.2T). Using (A.2S). (A.26) leads to the desired result:

$$
\int \frac{d \mathbf{k}}{(2 \pi)^{3}}\left[z-2 \epsilon_{k_{1}}+2 \mu+i \eta\right] \mathrm{K}^{0}\left(\mathbf{k} \cdot \mathbf{k}^{\prime} \cdot \mathbf{K}: z\right) \Phi_{\mathbf{k}_{1}}^{z}(\mathbf{k})=\left(z-2 \epsilon_{k^{\prime}}+2 \mu\right) \Phi_{\mathbf{k}_{\mathrm{t}}}^{z}\left(\mathbf{k}^{\prime}\right)
$$

Since the factor $D \equiv\left(z-2 \epsilon_{k_{1}}+2 \mu+i \eta\right)$ is independent of the integral variable k. we can take it outside of the integral. We next multiply both side of (A.29) by $D^{-1} \Phi_{\mathbf{k}_{1}}\left(\mathbf{k}_{2}\right)$. and integrate over $\mathbf{k}_{1}$. Using the completeness relation (A.9). one arrives at

$$
\gamma^{0}\left(\mathbf{k}_{2}, \mathbf{k}^{\prime}, \mathbf{K} ; z\right)=\left(z-2 \epsilon_{k^{\prime}}+2 \mu\right) \int \frac{d \mathbf{k}_{1}}{(2 \pi)^{3}} \frac{\phi_{\mathbf{k}_{1}}^{z}\left(\mathbf{k}^{\prime}\right) \phi_{\mathbf{k}_{1}}\left(\mathbf{k}_{2}\right)}{z-2 \epsilon_{k_{1}}+2 \mu}
$$


Esing the complex conjugate of (A.6) in (A.30). one finds

$$
\begin{aligned}
l^{\circ}\left(\mathbf{k}_{2}, \mathbf{k}^{\prime} \cdot \mathbf{K}: z\right)=\sigma_{\mathbf{k}^{\prime}}\left(\mathbf{k}_{2}\right)+\int \frac{d \mathbf{k}_{1}}{(2 \pi)^{3}} \dot{\omega}_{\mathbf{k}_{1}}\left(\mathbf{k}_{2}\right)\left[\frac{1}{z-2 \epsilon_{k_{1}}+2 \mu}\right. \\
\left.+\frac{1}{2 \epsilon_{k_{1}}-2 \epsilon_{k^{\prime}}-i \eta_{i}}\right] \tilde{f}^{\approx}\left(\mathbf{k}_{\mathbf{1}}, \mathbf{k}^{\prime}\right) .
\end{aligned}
$$

We now multiply (A.31) by $v\left(k-k_{2}\right)$ and integrating over $\mathbf{k}_{2}$. The definition of $\backslash^{0}$ and $\Gamma^{0}$ in (A.24) and (A.25) plus (A.6) leads to

$$
\begin{aligned}
\Gamma^{0}\left(\mathbf{k} \cdot \mathbf{k}^{\prime} \cdot \mathbf{K}: z\right)=\dot{f}\left(\mathbf{k}, \mathbf{k}^{\prime}\right)+\int \frac{d \mathbf{q}}{(2 \pi)^{3}} \dot{f}(\mathbf{k}, \mathbf{q})\left[\frac{1}{z-2 \epsilon_{q}+2 \mu}\right. \\
\left.+\frac{1}{2 \epsilon_{q}-2 \epsilon_{k^{\prime}}-i \eta}\right] \dot{f}^{*}\left(\mathbf{k}^{\prime} \cdot \mathbf{q}\right) .
\end{aligned}
$$

This last expression has the important feature that it gives $\Gamma^{0}$ completely in terms of the free space (or vacuum) scattering amplitude $\bar{f}$.

We are now in position to express the full scattering amplitude $\Gamma$ in the medium. as given by (A.18). in terms of $\dot{f}$. A slight rearrangement of (A.23) yields

$$
\begin{gathered}
\imath\left(\mathbf{k} \cdot \mathbf{k}^{\prime} \cdot \mathbf{K}: z\right)-\frac{1}{z-2 \epsilon_{k}+2 \mu} \int \frac{d \mathbf{q}}{(2 \pi)^{3}} \gamma\left(\mathbf{k}-\mathbf{q} \cdot \mathbf{k}^{\prime} \cdot \mathbf{K}: z\right) \\
\quad=(2 \pi)^{3} \delta\left(\mathbf{k}-\mathbf{k}^{\prime}\right)+\frac{F_{+}(\mathbf{K} \cdot \mathbf{k})-1}{2-2 \epsilon_{k}+2 \mu} \Gamma\left(\mathbf{k} \cdot \mathbf{k}^{\prime} \cdot \mathbf{K}: z\right) .
\end{gathered}
$$

Comparing this with (A.26) divided by $z-2 \epsilon_{k}+2 \mu$ shows that the operator on the left side of (A.33) is just the inverse of $x_{0}$. which means $\chi$ can be expressed in terms of 10 as follows:

$$
\begin{aligned}
\curlywedge\left(\mathbf{k}, \mathbf{k}^{\prime}, \mathbf{K} ; z\right)=\quad \chi_{0}\left(\mathbf{k}, \mathbf{k}^{\prime} . \mathbf{K} ; z\right)+\int \frac{d \mathbf{q}}{(2 \pi)^{3}} \gamma_{0}(\mathbf{k}, \mathbf{q}, \mathbf{K} ; z) \\
\quad \times\left[\frac{F_{+}(\mathbf{K}, \mathbf{q})-1}{z-2 \epsilon_{q}+2 \mu}\right] \Gamma\left(\mathbf{q}, \mathbf{k}^{\prime}, \mathbf{K} ; z\right) .
\end{aligned}
$$

Taking the convolution with $v$ in (A.34), we find

$$
\Gamma\left(\mathbf{k}, \mathbf{k}^{\prime}, \mathbf{K}: z\right)=\Gamma^{0}\left(\mathbf{k}, \mathbf{k}^{\prime}, \mathbf{K}, z\right)+\int \frac{d \mathbf{q}}{(2 \pi)^{3}} \Gamma^{0}(\mathbf{k}, \mathbf{q}, \mathbf{K}: z)
$$




$$
\times\left[\frac{F_{+}(\mathbf{K}, \mathbf{q})-1}{z-2 \epsilon_{k}+2 \mu}\right] \Gamma\left(\mathbf{q}, \mathbf{k}^{\prime} \cdot \mathbf{K}: z\right)
$$

Equation (A.35) expresses $\Gamma$ in term of the reduced scattering amplitude $\Gamma^{0}$, while $\Gamma^{0}$ in turn can be expressed in terms of the free space scattering amplitude $\dot{f}$ using (A.32). In other words, through the intermediate function, $\Gamma^{0}$, we can relate $\Gamma$ directly to $f$. This result is important because it enables us to deal with an interatomic potential with a hard core. In that case, the iteration of the integral equation (A.11) for $\Gamma$ in powers of $v$ makes only formal sense, since each term is large. In contrast. (A.32) and (A.35) show that $\Gamma$ is well-defined as long as $\dot{f}$ is. 


\section{Bibliography}

[1] M. H. Anderson. J. R. Ensher. M. R. Matthews. C. E. Wieman, and E. A. Cornell. Science 269, 198 (1995).

[2] K. Burnett. Contemporary Physics 37. 1 (1996).

[3] Bose-Einstein Condensation. edited by A. Griffin. D. W. Snoke. and S. Stringari (Cambridge Lniversity Press. New York. 1995).

[4] A. Einstein. Sitzungsber. Kgl. Preuss. Akad. Wiss. 3 (1925).

[5] K. Huang, Statistical Mechanics. 2nd ed. (John Wiley. New York. 198i).

[6] F. London. Nature 141. 643 (1938).

[7] L. D. Landau, J.Phys. L.S.S.R. 5. 71 (1941).

[8] A. Griffin. Excitations in a Bost-Condensed Liquid (Cambridge Cniversity Press. New York. 1993).

[9] N. N. Bogoliubov, J. Phys. L.S.S.R. 11. 23 (1947).

[10] O. Penrose and L. Onsager. Phys. Rev. 104. 5i6 (1956).

[11] S. T. Beliaev, Soviet Phys. -JETP 7. 289 (19.58).

[12] A. A. Abrikosov, L. P. Gorkov, and I. E. Dzyaloshinski. Methods of Quantum Field Theory in Statistical Physics (Prentice-Hall, Englewood Cliffs. N..J., 196:3).

[13] A. L. Fetter and J. D. Walecka, Quantum Theory of Many-Particle Systems (McGraw-Hill, New York, 1971). 
[14] J. C. Inkson. Many-Body Theory of Solids (Plenum. New York. 1984).

[1.5] H. Shi. G. Verechaka, and A. Griffin. Phys. Rev. B 50. 1119 (1994).

[16] J. L. Lin and J. P. Wolfe. Phys. Rev. Lett 71. 1223 (1993).

[17] G. Kavoulakis. G. Baym. and J. P. Wolfe, Phys. Rev B53, $72 \cdot 27$ (1996).

[18] K. B. Davis. M. O. Mewes. M. R. Andrews. N. J. van Druten. D. S. Durfee. D. M. Kurn. and W. Ketterle. Phys. Rev. Lett. 75. 3969 (1995).

[19] .. Edwards. P. A. Ruprecht. K. Burnett. R. J. Dodd. and C. W. Clark. Phys. Rev. Lett. 77. 1671 (1996).

[20] E. P. Gross. Nuovo Cimento 20. 454 (1961).

[21] L. P. Pitaevskii. Sov. Phys. -.JETP 13. 451 (1961).

[22] J. Gavoret and P. Nozières. Ann. Phys. (N.Y.) 28. 349 (1964).

[2:3] N. M. Hugenholtz and D. Pines. Phys. Rev. 116, 489 (1959).

[24] T. D. Lee. K. Huang, and C. N. Yang. Phys. Rev. 106. 1135 (195i): K. Huang. and C. N. Yang, and J. M. Luttinger. Phys. Rev. 105. Ti6 (195i): K. Huang in Studies in Statistical Mechanics, vol.2. J. De. Boer and G.E. Thlenbeck. eds. (North-Holland. Amsterdam, 1964).

[25] K. Huang. Statistical Mechanics. Ist ed. (John Wiley, New York, 196:3). Chap. 13.

[26] P. C. Hohenberg and P. C. Martin. Ann. Phys. (N.Y.) 34. 291 (1965).

[2T] V. N. Popov and L. D. Faddeev, Sov. Phys. -JETP 20. 890 (1965).

[28] K. K. Singh, Physica 34. 285 (1967).

[29] T. H. Cheung and A. Griffin. Phys. Rev. A 4, 237 (1971).

[30] Y. A. Nepomnyashchii and A. A. Nepomnyashchii, Sov. Phys. -.JETP 48. 49:3 (1978). 
[31] V. N. Popov, Functional Integrals in Quantum Field Theory and Statistical Physics (Reidel. Dordrecht. 1983). Chap. 6.

[32] V. N. Popov. Soviet Phys. -JETP 20. 118.5 (1965).

[33] M. Bijlsma and H. T. C. Stoof. Phys. Rev. A 55. 498 (1997).

[3-4] S. T. Beliaev, Soviet Phys. -JETP 7, 299 (1958).

[35] G. D. Mahan. Many-Particle Physics (Plenum Press, New York. 1981).

[36] E. M. Lifshitz and L. P. Pitaevskii, Statistical Physics. Part 2 (Pergamon. New York. 1980).

[3i] E. F. Talbot. Some Rigorous Results in the Theory of Bose liquids (Ph.D Thesis. Lniversity of Toronto. 198:3).

[38] Y. Nambu. Phys. Rev. 117, 648 (1960).

[:39] L. P. Kadanoff and G. Baym, Quantum Statistical Mechanics (W. A. Benjamin. Inc. New York. 1962).

[40] A. E. Glassgold. A. N. Kaufman. and K. .I. Watson. Phys. Rev. 120. 660 (1960).

[4l] A. Griffin. Phys. Rev. B 53. 9341 (1996).

[42] A. Griffin. private communication.

[+3] K. Kehr. Z. Phys. 221, 291 ( 1969).

[44] L. Reatto and J. P. Straley. Phys. Rev. 183, 321 (1969).

[45] M. Bijlsma and H. T. C. Stoof, Phys. Rev. A 54, 5085 (1996).

[46] P. Nozières, Theory of Interacting Fermi Systems (W.A. Benjamin, New York. 1964).

[47] The following analysis developed from discussion with Dr. H. T. C. Stoof. 
[48] A. A. Nepomnyashchii and Y. A. Nepomnyashchii. Sov. Phys. -JETP 38. 134 $(197 t)$

[49] H. Shi and A. Griffin, to be published.

[50] D. A. W. Hutchinson. E. Zaremba, and A. Griffin. Phys. Rev. Lett. 78, 18+2 (1997).

[51] C'. Castellani. C. Di Castro. F. Pistolesi, and C. C. Strinati. 1995 (preprint): Phys. Rev. Lett, in press.

[52] S. K. Ma and C. W. Woo. Phys. Rev. 159, 165 (1967).

[5:3] V. K. Wong and H. Gould. Ann. Phys. (N.Y.) 83, 252 (1974).

[.j4] E. Talbot and A. Griffin. Ann. Phys. (N.Y.) 151. il (198:3).

[5.5] L. I. Schiff, Quantum .Mechanics (.McGraw-Hill. New York. 1968).

[56] V. M. Galitskii and A. B. Migdal. Soviet Phys. -JETP 7. 96 (19.58). 

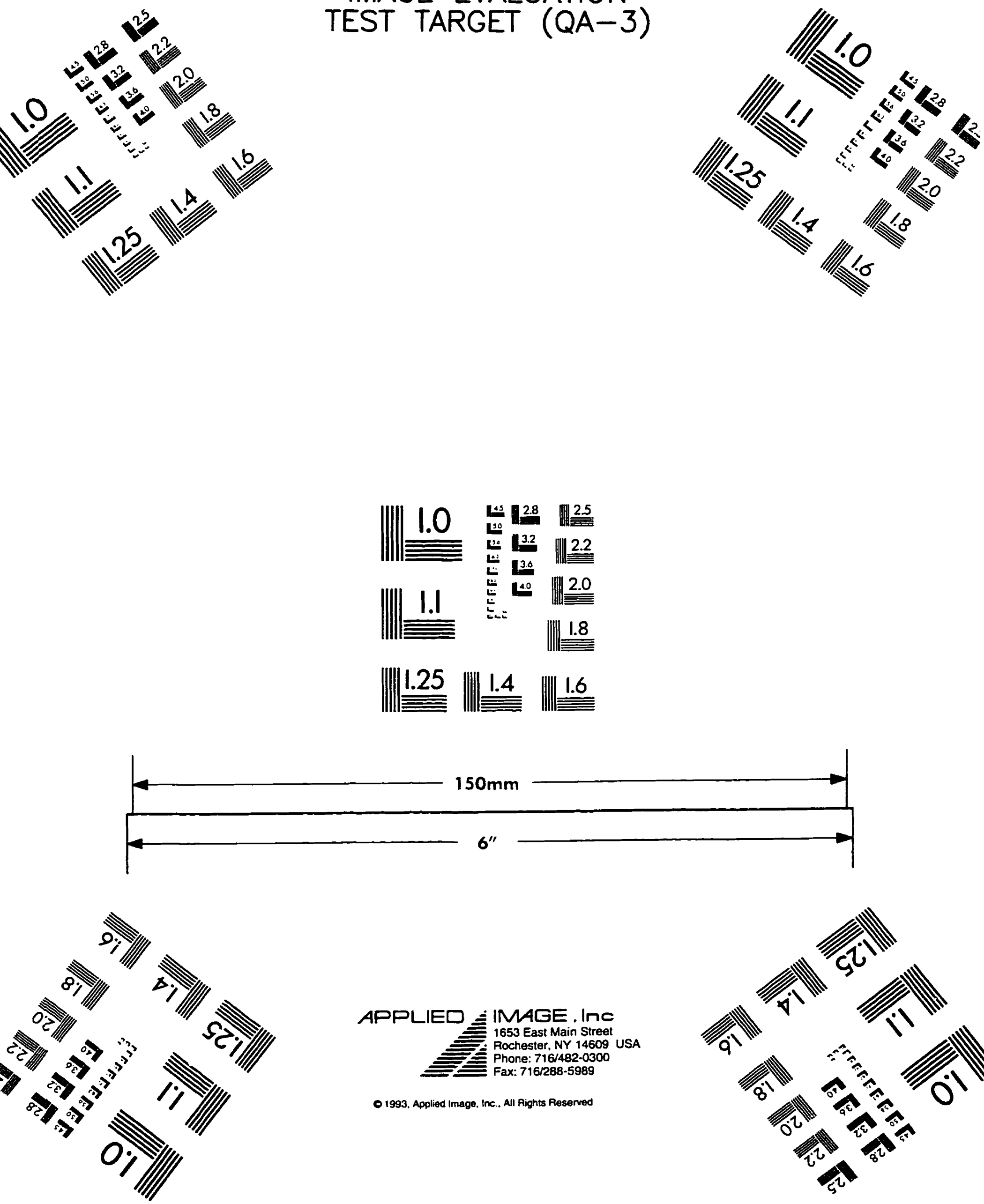

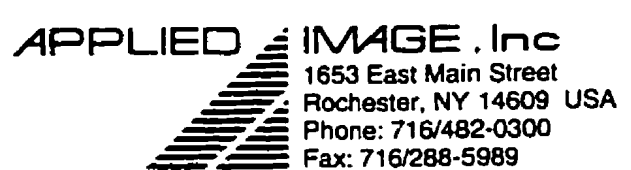

O 1993. Applied Image. Inc.. All Aights Reserved

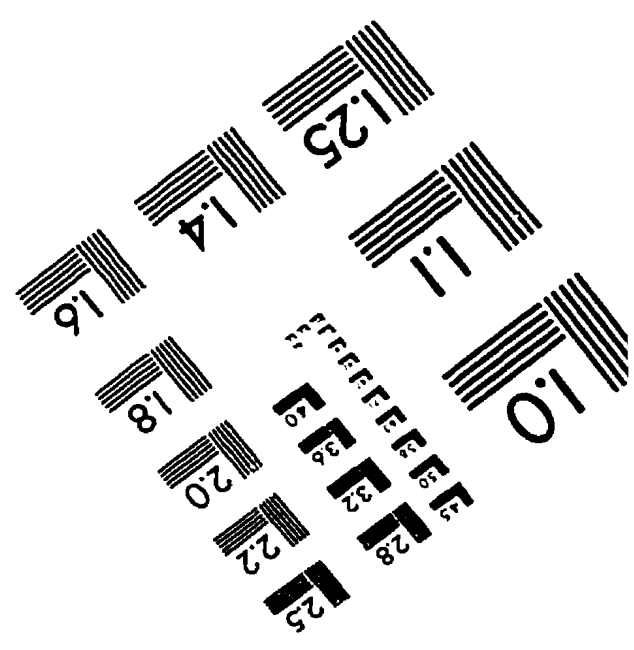

